\title{
FLOW-INDUCED VIBRATION
}

IN LMFBR STEAM GENERATORS:

A STATE-OF-THE-ART REVIEW

\section{by}

Y. S. Shin and M. W. Wambsganss

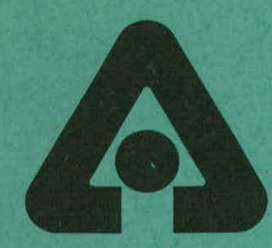

ARGONNE NATIONAL LABORATORY, ARGONNE, ILLINOIS

Prepared for the U.S. ENERGY RESEARCH

AND DEVELOPMENT ADMINISTRATION

Division of Reactor Research and Development

under Contract W-31-109-Eng-38 


\section{DISCLAIMER}

This report was prepared as an account of work sponsored by an agency of the United States Government. Neither the United States Government nor any agency Thereof, nor any of their employees, makes any warranty, express or implied, or assumes any legal liability or responsibility for the accuracy, completeness, or usefulness of any information, apparatus, product, or process disclosed, or represents that its use would not infringe privately owned rights. Reference herein to any specific commercial product, process, or service by trade name, trademark, manufacturer, or otherwise does not necessarily constitute or imply its endorsement, recommendation, or favoring by the United States Government or any agency thereof. The views and opinions of authors expressed herein do not necessarily state or reflect those of the United States Government or any agency thereof. 


\section{DISCLAIMER}

Portions of this document may be illegible in electronic image products. Images are produced from the best available original document. 
The facilities of Argonne National Laboratory are owned by the United States Government. Under the terms of a contract (W-31-109-Eng-38) between the U. S. Energy Research and Development Administration, Argonne Universities Association and The University of Chicago, the University employs the staff and operates the Laboratory in accordance with policies and programs formulated, approved and reviewed by the Association.

\section{MEMBERS OF ARGONNE UNIVERSITIES ASSOCIATION}

The University of Arizona Carnegie-Mellon University Case Western Reserve University The University of Chicago University of Cincinnati Illinois Institute of Technology University of Illinois Indiana University Iowa State University The University of Iowa
Kansas State University

The University of Kansas

Loyola University

Marquette University

Michigan State University

The University of Michigan

University of Minnesota

University of Missouri

Northwestern University

University of Notre Dame
The Ohio State University

Ohio University

The Pennsylvania State University

Purdue University

Saint Louis University

Southern Illinois University

The University of Texas at Austin

Washington University

Wayne State University

The University of Wisconsin

\section{NOTICE}

This report was prepared as an account of work sponsored by the United States Government. Neither the United States nor the United States Energy Research and Development Administration, nor any of their employees, nor any of their contractors, subcontractors, or their employees, makes any warranty, express or implied, or assumes any legal liability or responsibility for the accuracy, completeness or usefulness of any information, apparatus, product or process disclosed, or represents that its use would not infringe privately-owned rights. Mention of commercial products, their manufacturers, or their suppliers in this publication does not imply or connote approval or disapproval of the product by Argonne National Laboratory or the U. S. Energy Research and Development Administration.

Printed in the United States of America Available from

National Technical Information Service

U. S. Department of Commerce

5285 Port Royal Road

Springfield, Virginia 22161

Price: Printed Copy $\$ 5.45 ;$ Microfiche $\$ 2.25$ 


\section{ARGONNE NATIONAL LABORATORY \\ 9700 South Cass Avenue \\ Argonne, Illinois 60439}

\section{FLOW-INDUCED VIBRATION}

IN LMFBR STEAM GENERATORS:

A STATE-OF-THE-ART REVIEW

by

Y. S. Shin and M. W. Wambsganss

Components Technology Division

May 1975

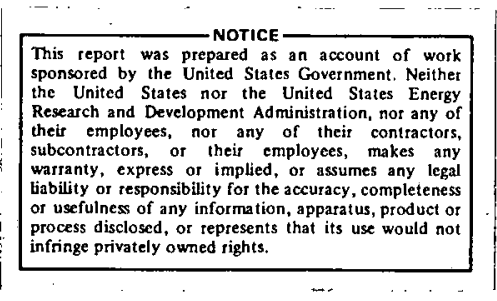




\section{PREFACE}

This report is prepared as a part of an AN.L Flowinduced Vibration Program in support of the Clinch River Breeder Reactor. Plant (CRBRP) steam-generator design activity.

In the U.S., several LMFBR steam generators have been operated. 'The designs of two of these units are reviewed along with the three CRBRP candidate designs. Failure experiences of the Enrico Fermi unit are also reviewed.

The interial components of a stcam generator that are exposed to fluid flow include the tubes, baffles, support members, shrouds, and flow distributors. Of these components, the tubes are the most susceptible to flow-induced vibration, as past experience with shell-and-tube heat exchangers has shown. Consequently, this report focuses on tube vibration. Excitation mechanisms and state-of-the-art technology relating to flowinduced vibration analysis/testing are reviewed and discussed. Although emphasis is on tube vibration, the information and methods presented are generally applicable to other components.

This report contains the information available fur making the best possible evaluation of potential flow-induced viluation in LMFBR steam generators. As a sequel, a design guide is planned with this information put into a format that permits a steam-generator designer to follow a step-by-step procedure lo evaluate a given design from the standpoint of flow-induced vibration. 
TABLE OF CONTENTS

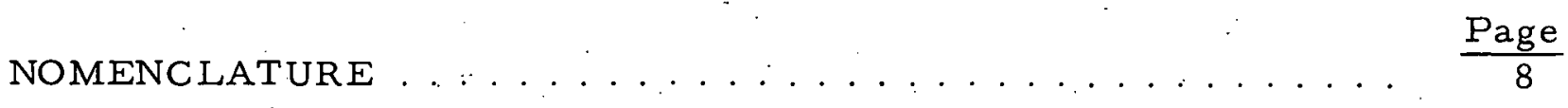

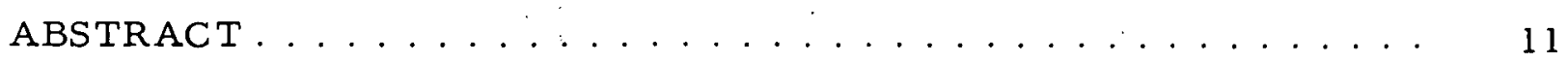

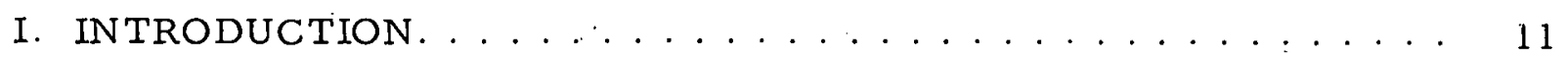

II. LMFBR STEAM-GENERATOR CONFIGURATIONS AND DESIGN

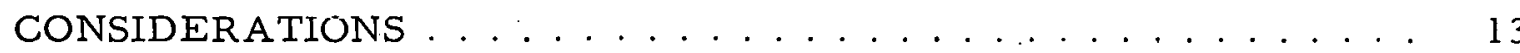

A. Configurations .......................... 14

1. Hockey-stick Concept . . . . . . . . . . . . . . . 14

2. Bayonet-tube Concept ................. 17

3. Duplex-J Tube Concept ................. 17

4. EBR-II Steam Generator . . . . . . . . . . . . . . 17

5. Enrico Fermi Atomic Power Plant Steam Generator . . . . . . 21

B. Design Conside rations . . . . . . . . . . . . . 21

1. Baffle Spacing . . . . . . . . . . . . . . . 21

2. Tube-baffle-hole Clearance .............. 24

3. Flow Distributor/Impingement Plate .......... 24

4. Vibration Suppressor. . . . . . . . . . . . . . 24

5. Sizing Inlet/Outlet. . . . . . . . . . . . . . . . 24

6. Baffle Thickness, Baffle-hole Shape ............ 25

7. Tube Spacing ........................ 25

8. Differential Thermal Expansion. ............ 25

9. Shell-side Flow Orientation ............. 25

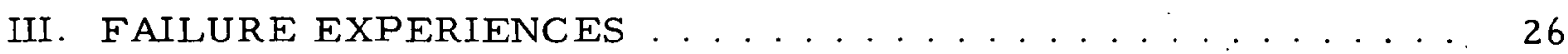

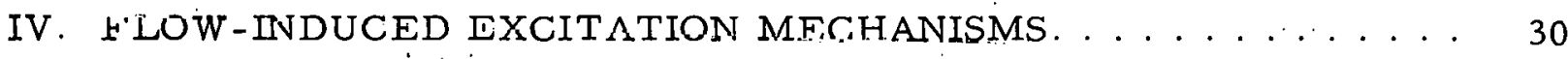

A. Vortex Shedding . . . . . . . . . . . . . . 30

1. Reynolds Number . . . . . . . . . . . . . . . . 31

2. Strouhal Number. . . . . . . . . . . . . . . . . 33

3. Strouhal Number as a Function of Tube Spacing ....... 34

4. Drag and Lift Forces.................. 35

5. Vibrating Tubes .................... 38

6. Acoustic Standing Waves ............... 40

B. Turbulent Buffeting .................... 41

C. Fluidelastic Whirling . . . . . . . . . . . . . . . 444 44

1. Connors' Threshold Velocity. ... . . . . . . . . . 44 


\section{TABLE OF CONTENTS}

$\underline{\text { Page }}$

D. Jet Switching . . . . . . . . . . . . . . . . 47

E. Turbulent Pressure Fluctuations in Parallel Flow . . . . . 48

F. Hydraulic Noise . . . . . . . . . . . . . . . . . . . 48

V. VIBRATION ANALYSIS. ... . . . . . . . . . . . . . 49

A. Shell-side Flow Velocities . . . . . . . . . . . . . 51

B. Added Mass. . . . . . . . . . . . . . . . . . 52

C. Natural Frequency of Tube Vibration. . . . . . . . . . . 54

1. Single-span Straight Beam. . . . . . . . . . . . 55

2. Singlo-opan Curved Beam ............... 56

3. Multiple-span Beams ................... 57

4. Effects of Tube-to-Baffle-hole Clearance and Baffle Thickness. . . . . . . . . . . . . . . . . . 60

5. Tube-baffle Impact. . . . . . . . . . . . . . . 62

D. Damage-number Concept ................66 62

E. Prediction of Subcritical Vibration Amplitude in Parallel Flow ........................ 64

1. Empirical Expression for Peak Amplitude . . . . . . . . 64

2. RMS Displacement Expression. . . . . . . . . . 66

VI. VIBRATION TESTING $\ldots \ldots \ldots \ldots \ldots \ldots \ldots . \ldots \ldots$

A. Model Testing . . . . . . . . . . . . . . . . . 70

B. Surveillance Methods . . . . . . . . . . . . . . . 75

VII. CONCLUDING REMARKS $\ldots \ldots \ldots \ldots \ldots$

A. Summary .......................... 76

B. Future Research Areas. . . . . . . . . . . . . . . . 77

ACKNOW LEDGMENTS . . . . . . . . . . . . . . . . . . . 80

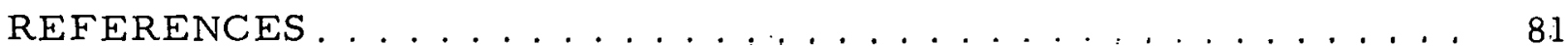




\section{LIST OF FIGURES}

No.

Title

$\underline{\text { Page }}$

1. Steam-generating System .................... 13

2. Hockey-stick Concept: AI Steam Generator : . . . . . . . . . . . 16

3. Bayonet-tube Concept: FW Steam Generator . . . . . . . . . 18

4. Duplex J-tube Concept: W Steam Generator........... 19

5. EBR-II Steam Generator (ANL) . . . . . . . . . . 20

6. Enrico Fermi Atomic Power Plant (EFAPP) Steam Generator . . 22

7. Plan View and Cross Section of EFAPP Steam Generator . . . . 23

8. EFAPP Steam Generator: Tube-wall Thinning Caused by Rubbing Inside of Support Bars. . . . . . . . . . . . . . 26

9. EFAPP Steam Generator: Broken Tubes at Support Bar due to Excessive Tube Vibration . . . . . . . . . . . . . . 27

10. EFAPP Steam Generator: Tube Crack due to Collision with

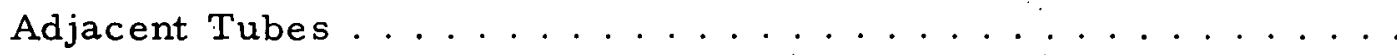

11. EFAPP Steam Generator: Enlargement of Support-bar Holes due to Tube-to-Support-bar Impacts . . . . . . . . . . . 28

12. EFAPP Steari Generator: Samples of Broken Tubes with "Knife-cut" Damage by Tube-to-Tube Collision. . . . . . . . . 28

13. Regimes of Fluid Flow across Rigid Circular Cylinder. . . . . . 32

14. Experimentàlly Determined Strouhal-Reynolds-number Relationship for Circular Cylinders .............. 33

15. Envelope of Strouhal-Reynolds-number Relationship for

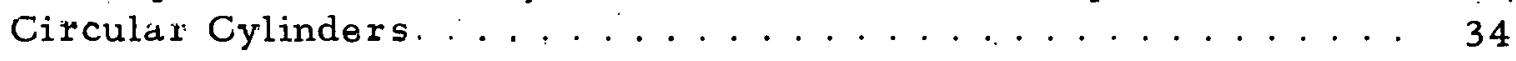

16. Tube Patterns. ...................... 35

17. Strouhal Number vs Transverse Tube-spacing Ratio with Longitudinal Tube-spacing Ratio for In-line Tube Banks . . . . . 36

18. Strouhal Number vs Transverse Tube-spacing Ratio with Longitudinal Tube-spacing Ralio for Staggered Tube Banks . . . 36

19. Drag and Lift Furces . . . . . . . . . . . . . . . 37

20. Drag Coefficients for Rigid Circular Cylinders: $0.4 \leq \operatorname{Re}<10^{3}$. 39

21. Drag Coefficients for Rigid Circular Cylinders: $10^{3} \leq \operatorname{Re}<10^{7}$. 39

22. Experimentally Determined Mean Oscillatory Lift Coefficients for a Single Stationary Cylinder . . . . . . . . . . . . . 40 


\section{LIST OF FIGURES}

No.

Title

Page

23. Typical One-dimensional Turbulent-energy Spectrum

Determined Experimentally. . . . . . . . . . . . . . .

24. Stability Diagram for Single-row Arrays Having a Pitch-toDiameter Ratio of 1.41 .

25. Stability Diagram for Tube Arrays . . . . . . . . . . . 46

26. Jet Pairing from a Single Row of Tubes . . . . . . . . . . . . 47

27. Tube in a Compressihle-fluid Annulus . . . . . . . . . . . 53

28. Added Mass Coefficients for Varinıs Rnd/Wall-diamctcr Raius

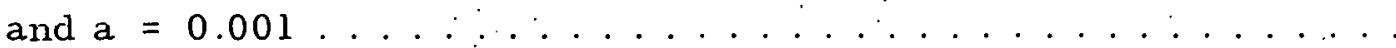

29. Added Mass Coefficients for Seven-t.ube Hexagonal Array alld Nine-tube Square Array. . . . . . . . . . . . . . . . . .

30. Frequency Factors for In-plane Vibration of Curved Beam with Three Conditions . . . . . . . . . . . . . . . .

31. Frequency Factors for Out-of-plane Vibration of Curved Beam

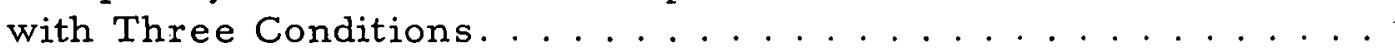

32. Effects of Tube-to-Baffle-hole Clearance on Natural Frequency

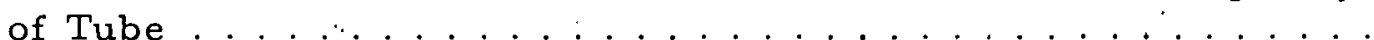

33. Hypothetical Curve of Tube Impact Velocity vs Tube-to-Baffle-

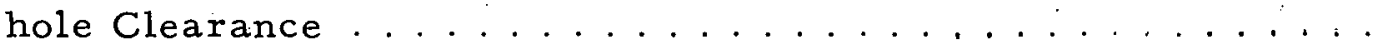

34. Agreement between Measured and Predicted Amplitudes of Vibration according to Paidoussis' Empirical Expression.

35. Equivalent Viscous Damping Factor as a Function of Mean Axial Flow Velocity for a 0.5-in.-dia, $46 \frac{7}{8}$-in.-long Brass Rod with

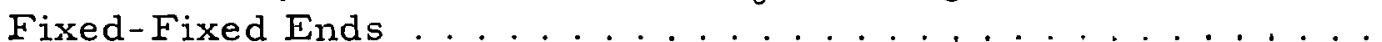

36. Family of Near-field Mean-square Wall-pressure Spectra as a Function of Strouhal Number ...................

37. Agreement between Chen and Wambsganss' Theoretical and Experimental Values of the Amplitude of Subcritical Vibration.

38. Fluid Density and Viscosity vs Temperature. . . . . . . . . 70 


\section{LIST OF TABLES}

No.

Title

Page

I. Steam-generator Operating and Design Data ......... 15

II. Added Mass Coefficient for Various Rod/Wall-diameter Ratios .

III. Frequency Factor of a Single-span Straight Beam with Three Type s of End Conditions . . . . . . . . . . . . . 56 
Symbol

A

$A_{\mathrm{m}} \quad$ Cross-sectional tube metal area $=$

Projected area per linear length of cylinder $\left(d^{2}-d_{i}^{2}\right) \pi / 4$

$\begin{array}{ll}\text { A } & \text { Average crossflow area } \\ \text { a } & \omega d / 2 c \\ \text { b } & \omega D / 2 c\end{array}$

$B_{t} \quad$ Baffle thickness

c Velocity of sound in fluid

$c_{s}$. Longitudinal velocity of sound in structure

$C_{D} \quad$ Drag coefficient based on time-averaged drag force

$\mathrm{C}_{\mathrm{D}_{\mathrm{f}}} \quad$ Drag coefficient based on friction drag force

$\mathrm{C}_{\mathrm{D}_{\max }}$

Drag coefficient based on maximum absolute values of drag force

$\mathrm{C}_{\mathrm{D}_{\mathrm{o}}} \quad$ Drag coefficient based on oscillatory drag force

$\mathrm{C}_{\mathrm{D}_{\mathrm{p}}} \quad$ Drag coefficient based on pressure drag force

$C_{D_{\text {rms }}}$ Drag coefficient based on rms drag force

$\mathrm{C}_{K_{\mathrm{D}}}$ Drag coefficient based on harmonic-dragforce oscillation about the mean

$C_{L}$ L.ift. coefficient based on time-averaged lift force

$\mathrm{C}_{\mathrm{M}} \quad$ Added maśs coefficient

$\vec{c}_{\mathrm{p}} \quad$ rms pressure coefficient

C.I Minimum gap between adjacent tubes

D Diameter of rigid duct to confine the fluid annulus

$\mathrm{D}_{\mathrm{B}} \quad$ Outer-diameter limit of tube bundle

d Outer diameter of tube (or cylinder)

$d_{h} \quad$ Hydraulic diameter

$d_{i} \quad$ Inner diameter of tube

$d_{s} \quad$ Inner diameter of cylindrical shell

E. Modulus of elasticity

F $\quad$ Force amplitude

\section{NOMENCLATURE}

Symbol

Description

$F_{\text {A }} \quad$ Axial force

FD Time-averaged drag force

$F_{D_{f}} \quad$ Frictional drag force

$F_{D_{p}} \quad$ Pressure drag force

$F_{L} \quad$ Time-averaged lift force

$\mathrm{f}_{\mathrm{a}}$

$f_{b}$

$f_{n}$

$f_{0}$

Distance between centerlines of successive tube rows; $=\mathrm{L}$ for in-line tube array, $=\mathrm{L} / 2$

\section{$\ell$}

$\mathrm{m}_{\mathrm{f}}$

$\mathbf{m}_{\mathbf{i}}$

$m_{0}$ for staggered tube array

Acoustic-standing-wave resonant frequency

Dominant turbulent buffeting frequency

Natural frequency. of nth mode of tube

Cutoff frequency

Vortex-shedding frequency

Fundamental natural frequency of tube

Shear modulus of elasticity

Gap between adjacent tubes

Gravitational constant

Moment of inertia of tube

Polar moment of inertia of tube

Bessel functions of first kind of order $i$

Turbulent buffeting constant

Cọnstant

Flow-condition constant

Proportionality factor for fluidelastic instability

$\left\{\begin{array}{l}\text { Longitudinal spacing of tube } \\ \text { Characteristic length }\end{array}\right.$

Length of the tube (or beam) span between tube (or beam) supports

Added mass of fluid external to tube per unit length

Mass of fluid inside tube per unit length.

Virtual mass of tube (or beam) per unit length $\left(=m_{t}+m_{f}+m_{j}\right)$ 
THIS PAGE

\section{WAS INTENTIONALLY LEFT BLANK}


NOMENCLATURE

\begin{tabular}{|c|c|c|c|}
\hline Symbol & Description & Symbol & Description \\
\hline$m_{t}$ & Mass of tube per unit length & $\alpha_{1}$ & First-mode beam eigenvalue of the tube \\
\hline $\mathrm{N}_{\mathrm{BD}}$ & Baffle-damage number & $\alpha_{\mathrm{m}}$ & Modal constant \\
\hline${ }^{N} C D$ & Collision-damage number & ? & $=m_{f} / m_{0}$ \\
\hline $\mathrm{p}$ & Pitch of tubes & $\beta_{1}$ & Tube-to-baffle-hole clearance factor \\
\hline $\mathbf{R}$ & Radius of curvature & $\beta_{\mathrm{n}}$ & $\begin{array}{l}\text { Distortion of nth } \pi \text { term (similitude } \\
\text { requirement) }\end{array}$ \\
\hline $\mathbf{r}$ & $\begin{array}{l}\text { Stiffness ratio between torsional and bending } \\
\text { rigidity of beam }\end{array}$ & $\Gamma$ & Dimensionless axial force \\
\hline $\mathrm{Re}$ & Reynolds number & $\Delta$ & $\begin{array}{l}\text { Maximum transverse vibration amplitude of } \\
\text { tube at midspace }\end{array}$ \\
\hline $\mathrm{S}_{\mathrm{m}}$ & $\begin{array}{l}\text { Maximum allowable fatigue stress } \\
\text { (ASME Pressure Vessel Code, Sec. III) }\end{array}$ & $\vdots 6$ & Tube-to-baffle-hole clearance \\
\hline $\mathrm{S}_{\mathrm{v}}$ & Strouhal number & $\delta_{0}$ & Logarithmic decrement of tube in still fluid \\
\hline $\mathrm{T}$ & Transverse spacing of tube & $\delta_{1}$ & Correction factor \\
\hline $\mathrm{U}$ & Mean axial flow velocity & $\epsilon$ & $\ell / \mathrm{d}$ \\
\hline $\mathrm{U}_{0}$ & Flow velocity of fluid & 6 & Damping factor \\
\hline u & $\begin{array}{l}\text { Dimensionless mean axial flow velocity }= \\
\left(m_{f} / E I\right)^{1 / 2} U l\end{array}$ & $\varsigma_{1}$ & First-mode damping factor \\
\hline $\mathrm{V}$ & Free-stream velocity & $\eta$ & Poisson's ratio \\
\hline $\mathrm{V}_{1}$ & Mean flow velocity between adjacent tubes & & Subtended angle of curved beam \\
\hline $\mathrm{V}_{0}$ & Mean shell-side velocity & $\lambda_{n}$ & Frequency factor of nth mode \\
\hline $\mathrm{V}_{\mathrm{T}}$ & Threshold velocity for fluidelastic instability & $\mu$ & Viscosity of fluid \\
\hline$v_{i}$ & $\begin{array}{l}\text { Inpdel veloclly between lube dind lube } \\
\text { support }\end{array}$ & $\pi_{n}$ & $\begin{array}{l}\text { Kinematic viscosity of fluid } \\
\text { nth similitude requirement }\end{array}$ \\
\hline $\mathrm{W}$ & Shell-side mass flow rate. & $\rho$ & Mass density of fluid \\
\hline $\mathrm{x}$ & Support conditions of beam; both ends fixed & $\rho_{0}$ & Weight density of fluid \\
\hline $\mathbf{x}$ & Distance along the beam axis from support & $\rho_{\mathrm{s}}$ & Structural mass density \\
\hline$x_{l}$ & Longitudinal tube-spacing ratio $(\mathrm{L} / \mathrm{d})$ & $\left(\sigma_{\mathrm{y}}\right)_{\mathrm{rms}}$ & Root-mean-square bending stress of tube \\
\hline$x_{t}$ & Transverse tube-spacing ratio $(\mathrm{T} / \mathrm{d})$ & $\Phi_{0}$ & $\begin{array}{l}\text { Intensity of near-field mean-square spectral } \\
\text { density of pressure in low-frequency range }\end{array}$ \\
\hline$Y_{i}$ & Bessel function of the second kind of order $i$ & ${ }^{\Phi} \mathbf{p}$ & $\begin{array}{l}\text { Mean-square spectral density of near-field } \\
\text { wall pressure }\end{array}$ \\
\hline$y_{\text {rms }}$ & $\begin{array}{l}\text { Root-mean-square transverse displacement } \\
\text { of tube }\end{array}$ & $\begin{array}{l}\varphi_{1}(\mathbf{x}) \\
\omega\end{array}$ & $\begin{array}{l}\text { Fundamental mode of beam } \\
\text { Vibration frequency }\end{array}$ \\
\hline $\mathrm{z}$ & $\begin{array}{l}\text { Support conditions of beam; both ends } \\
\text { simply supported }\end{array}$ & $\omega_{0}$ & Frequency of driving force \\
\hline & onstant for average crossflow area & & \\
\hline
\end{tabular}


FLOW-INDUCED VIBRATION
IN LMFBR STEAM GENERATORS:

A STATE-OF-THE-ART REVIEW

by

Y. S. Shin and M. W. Wambsganss

\begin{abstract}
This state-of-the-art review identifies and discusses existing methods of flow-induced vibration analysis applicable to steam generators, their limitations, and base-technology needs. Also included are discussions of five different LMFBR steam-generator configurations and important design considerations, failure experiences, possible flow-induced excitation mechanisms, vibration testing, and available methods of vibration analysis. The objectives are to aid LMFBR steam-generator designers in making the best possible evaluation of potential vibration in steam-generator internals, and toprovide the basis for development of design guidelines to avoid detrimental flow-. induced vibration.
\end{abstract}

\title{
I. INTRODUC'TION
}

As part of ANL's flow-induced vibration program in support of the CRBRP steam-generator design activity, this state-of-the-art review identifies and discusses existing methods of flow-induced vibration analysis applicable to steam generators, their limitation, and base-technology needs. Also included are discussions of five different LMFBR steam-generator configurations and important design considerations, failure experiences, possible flowinduced excitation mechanisms, vibration testing, and available methods of vibration analysis. The objectives are to aid LMFBR steam-generator designers in making the best possible evaluation of potential vibration in steamgenerator internals, and to provide the basis for development of design guidelines to avoid detrimental flow-induced vibrations.

It is reported ${ }^{l}$ that operating heat exchangers have experienced damage due to excessive flow-induced vibration. This indicates that either existing techniques are inadequate to satisfactorily evaluate the potential for flowinduced vibration or that the state-of-the-art technology is not available in a form readily interpretable by and useful to the designer; it is suspected that the root cause for the occurrence of these vibration problems can be attributed, in part, to each of these elements. Many problems still exist in understanding and modeling fluid-flow phenomena and fluid/structure interaction. 
In structural dynamics, vibration problems are usually solved by applying known forcing functions. However, in flow-induced vibration, for example, of tube arrays in a steam generator, the vibration mechanisms themselves are often not clearly understood, with mathematical characterizations lacking or subject to uncertainties; these, in turn, are reflected in vibrationresponse predictions. An additional complication is related to the random nature of many of the fluid excitation forces.

Due to complexities of the flow field within a steam generator and the dependence of component vibration characteristics on fluid/structure interaction, scale-model tests are relied upon to verify design adequacy: The similitude requirements for valid dynamic model tests are presented. However, since all similitude requirements cannot be satisfied simultaneously, the model tests are necessarily distorted. To analytically account for the distortion requires an in-depth knowledge of the phenomena involved, and/or knowledge of the functional relationship among the similitude requirements describing a particular phenomenon.

The amount of published information on flow-induced vibration and related fluid-flow phenomena is increasing rapidly; an annotated bibliography on flow-induced vibrations ${ }^{2}$ includes over 350 references. In addition, several other reports and critical review papers in specific areas are available in the literature. ${ }^{1,3-6}$ 


\section{LMFBR STEAM-GENERATOR CONFIGURATIONS} AND DESIGN CONSIDERATIONS

The steam generator is one of the most important intermediate system components in an LMFBR power plant. This component transfers thermal energy from sodium to water to produce high-pressure and high-temperature steam to operate the turbines. A typical schematic loop layout of a sodiumheated steam generator system is shown in Fig. $1 .^{7}$ This system represents one of the candidate components for the Clinch River Breeder Reactor Plant (CRBRP), a 350-MWe Demonstration Plant being built near Oak Ridge, Tennessee. Sodium is circulated from the intermediate heat exchanger (IHX) through the superheater, evaporators, pump, and then back to the IHX. The evaporators use a recirculation pump to receive water from the steam drum. The water is partially evaporated with a steam quality of up to $50 \%$. The twophase mixture from the evaporator is then returned to the steam drum, where the water and steam are separated by steam dryers. The saturated steam is extracted from the top of the steam drum and superheated in the superheater. The superheated steam $\left(905^{\circ} \mathrm{F}, 1525 \mathrm{psia}\right)$ is then introduced into the turbines.

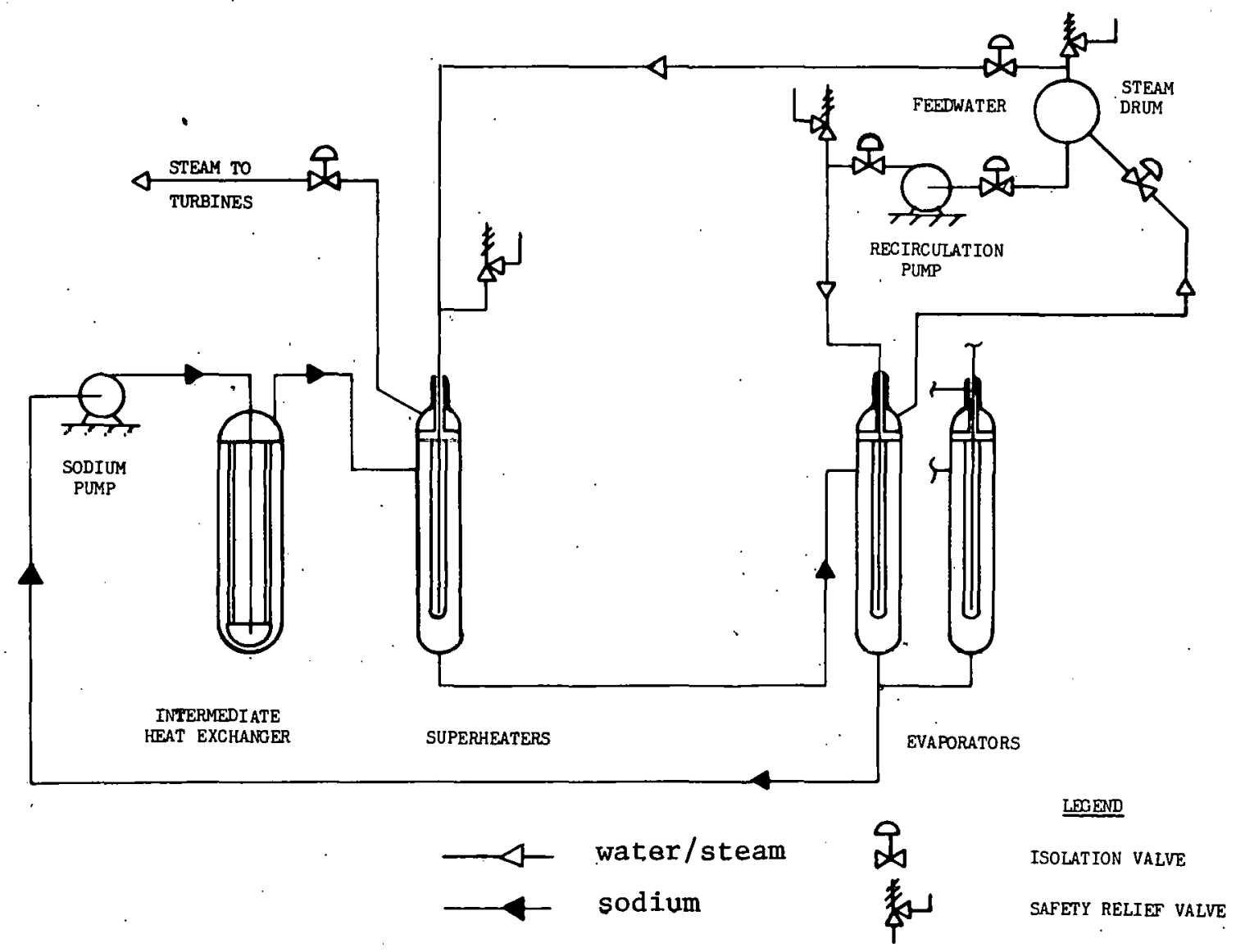

Fig. 1. Steam-generating System. (From Ref. 7.)

Flow-induced vibration problems are not peculiar to a specific type of steam generator. However, this report is directed to the LMFBR steam generator. Within the current LMFBR program, liquid sodium is on the shell side, 
transferring thermal energy to the water/steam on the tube side. However, the sodium-in-tube concept has also been examined. ${ }^{8}$ Since the sodium-water reaction is a safety problem of great concern in steam-generator design, the sodium-in-tube concept has an advantage over the more conventional arrangement in the sense that, if a tube leaks, the sodium-water reaction, by design, would be confined inside the failed tube without affecting the adjacent tubes.

\section{A. Configurations}

Several different configurations of steam generators exist. ${ }^{9-11}$ However, the primary goal of high reliability, safety, and maintainability is common to all types. Five different LMFBR steam-generator configurations have been selected to illustrate the basic mechanical arrangements:

1. Hockey-stick concept.

2. Bayonet-tube concept.

3. Luplex-J-tube concept.

4. EBR-II steam generator.

5. Enrico Fermi steam generator.

The first three units are the candidate steam generators for the CRBR. Demonstration Plant and are in various stages of design development. The EBR-II steam generator has been in operation since 1963 and has not experienced any vibrational problems. The Enrico Fermi units were first operated in 1962, subsequently encountering extensive tube damage caused by vibration. The pertinent design and operating data of the five selected configurations are listed in Table I. All units are vertically oriented to minimize thermal stratification. The tube material is $2 \frac{1}{4} \% \mathrm{Cr}-1 \% \mathrm{Mo}$ steel, and the tubes are unfinned. The evaporators and superheaters have identical mechanical arrangements except for size and material thickness.

\section{Hockey-stick Concept (Fig. 2)}

This unit, being developed by Atomics International, has singlewalled tubes intermediately supported by baffles, with both ends of the tubes welded to tubesheets. The tube bundle is surrounded by a shroud to promote uniform flow distribution. The shell encloses the entire internals. Thermal expansion of the tubes is accommodated by the bent-tube region. Vibration suppressors are placed in this region to eliminate potential low-frequency high-amplitude response; their utility was confirmed by test. ${ }^{15}$

a. Evaporator. Water enters the inlet plenum, flows up through the heat-transfer tube, and is heated by the sodium on the shell side. Sodium enters the shell side through the inlet nozzles, impinges on a flow distributor, is deflected circumferentially around the tube bundle, and flows down through the baffle holes and tube/baffle-hole clearances, over the tubes in axial flow, and exits through the outlet nozzle. 
(From Refs. 10, 12, 13, and 14)

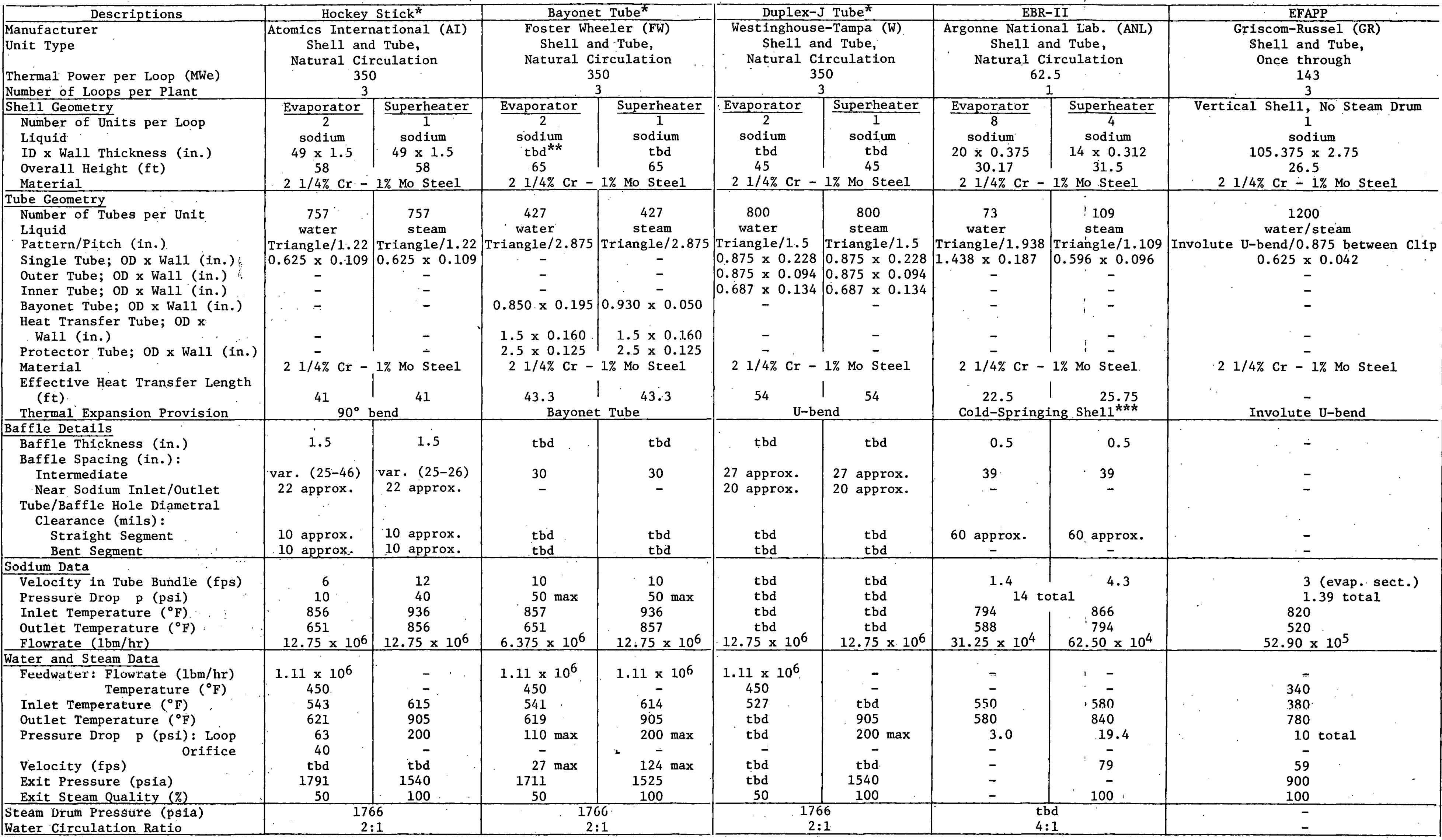

* Data shown are preliminary

${ }^{* *}$ tbd $=$ to be determined

${ }^{* * *}$ The shell was heated and elongated approximately $1 / 8$ in. while the tubes were being welded to tubesheets. 


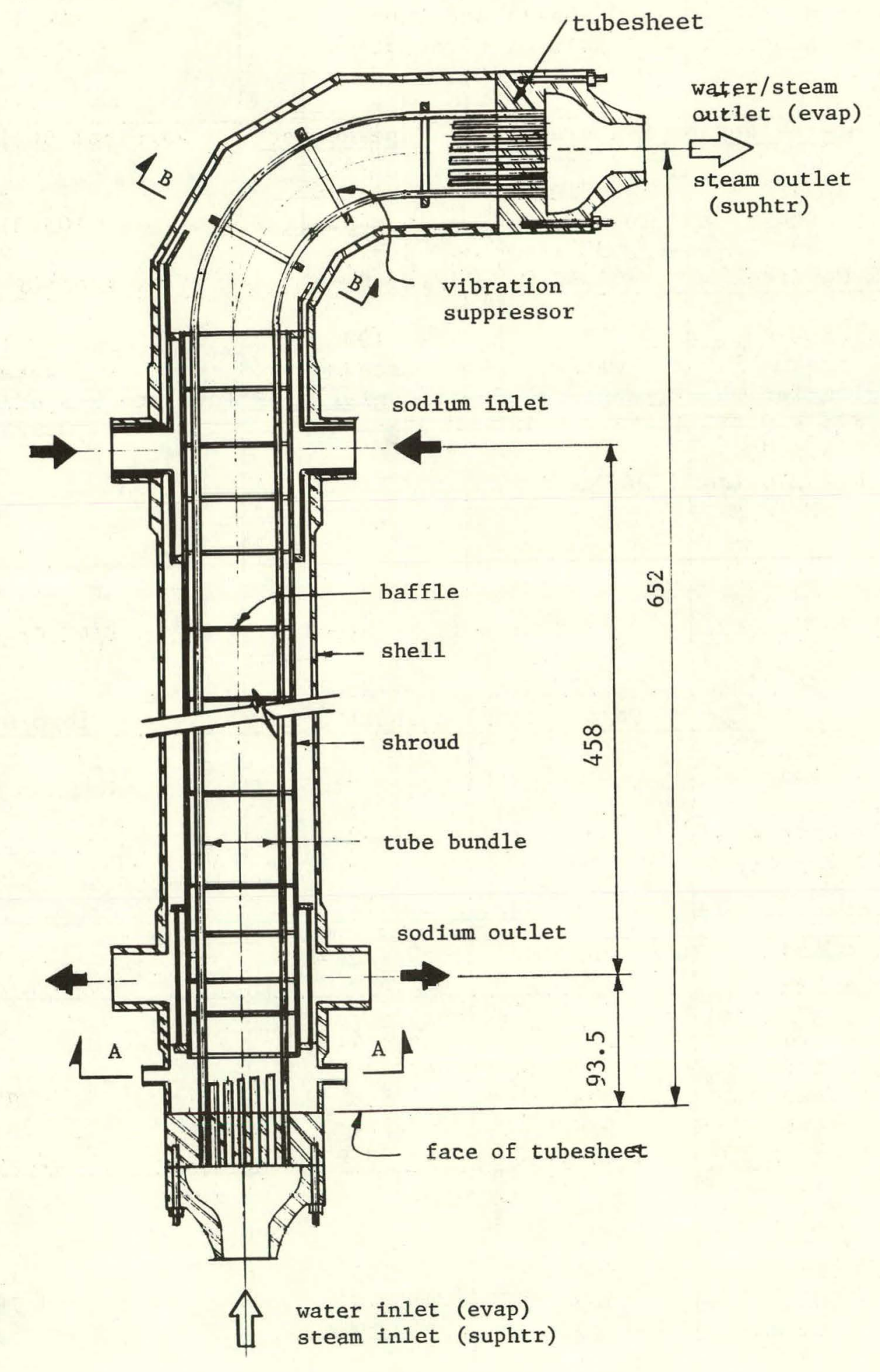

(a) Evaporator and Superheater

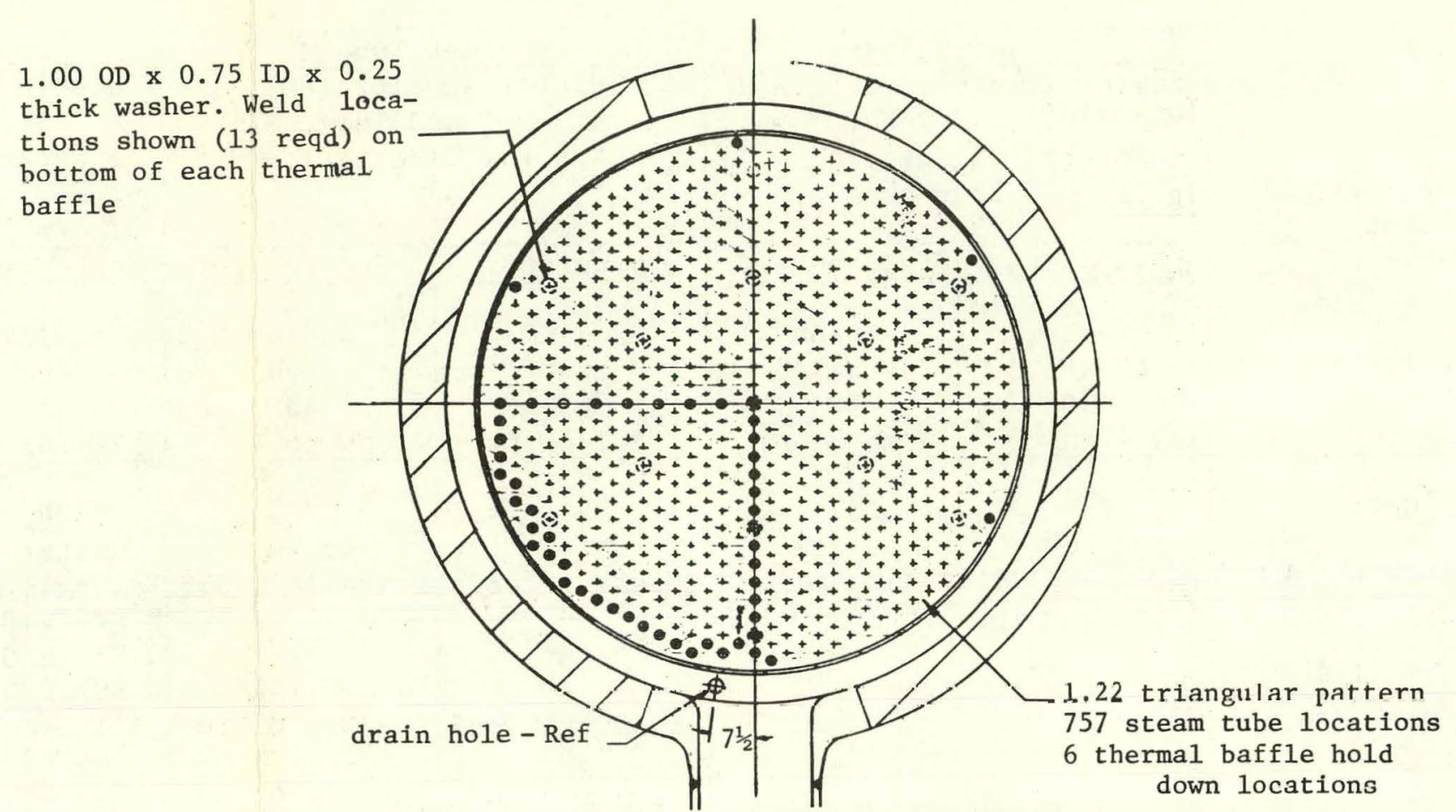

(b) Section A-A

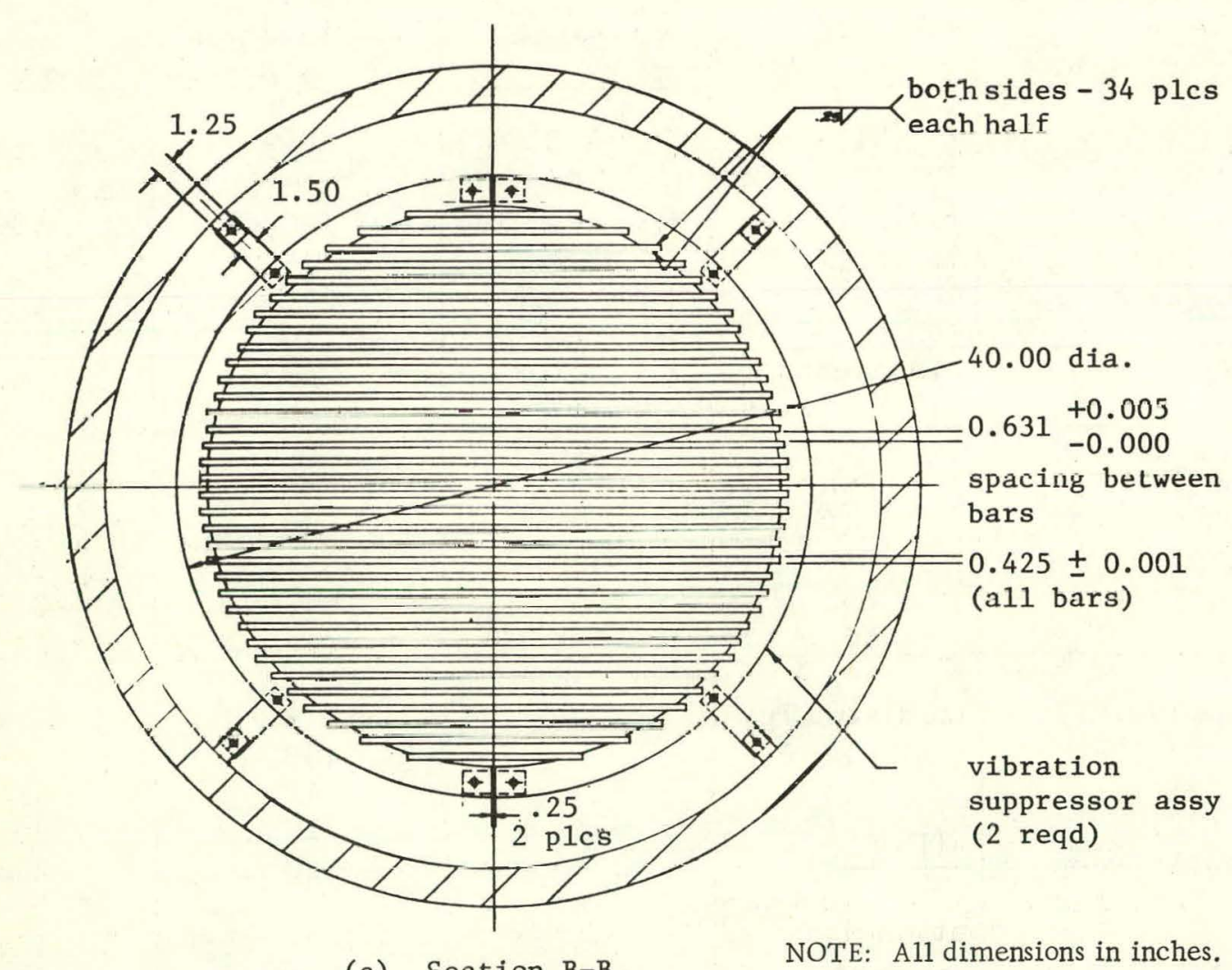

(c) Section $\mathrm{B}-\mathrm{B}$

NOTE: All dimensions in inches.

ig. 2. Hockey-stick Concept: AI Steam Generator. (Used with permission of Atomics International.) 
b. Superheater. Saturated steam enters the inlet plenum, flows up through the tube, and is superheated by the sodium on the shell side. The superheated steam is introduced to the turbines.

\section{Bayonet-tube Concept (Fig. 3)}

This unit has been proposed by Foster Wheeler Corporation. Each tube set consists of a bayonet tube, a heat-transfer tube, a protector tube, and an insulation tube (superheater only). The protector tube is intermediately supported by baffles, and both ends are welded to tubesheets. The shell encloses the entire internals. An advantage of the bayonet-tube concept is that the tubes are free to grow axially.

a. Evaporator. Water enters the inlet plenum, flows down through the bayonet tubes to the bottom of the heat-transfer tubes, and is heated by the sodium on the shell side in its upward pass in the annuli formed between the bayonet tubes and the heat-transfer tubes. Sodium enters the shell side through the inlet nozzle and impinges on the impingement plate, which deflects the sodium flow circumferentially around the inlet plenum.

b. Superheater. Saturated steam enters the outer plenum, flows down the central bayonet tube to the bottom of the heat-transfer tube, and then turns and flows up the annulus, where it is superheated by the sodium.

\section{Duplex-J Tube Concept (Fig. 4)}

Proposed by Westinghouse-Tampa, this unit has double-walled tubes with an annular gap for leak detection. The tubes are intermediately supported by baffles, with $\mathrm{U}$-bend vibration supports and tubesheets at the extreme ends. The duplex tubes are used to minimize the potential for a sodiumwater reaction. The shell encloses the entire internals.

a. Evaporator. Water enters the inlet plenum and flows down through the duplex tubes and U-bends, and up through the outlet plenum. Sodium enters the shell side through the inlet nozzle, impinges on a flow distributor, and flows circumferentially around the tube bundle and through the baffle holes.

b. Superheater. Saturated steam and sodium enter the inlet plenum and inlet nozzle respectively, and circulate as in the evaporator.

\section{EBR-II Steam Generator (Fig. 5)}

Developed by Argonne National Laboratory and built in Idaho, the EBR-II steam generator has been in operation since 1963 without experiencing any vibration problems. The unit has double-walled straight tubes with no annular gap, intermediately supporled by baffles, with a tuluesheet at each cnd. The duplex tubes at the sodium inlet are covered with shock tubes for protection 


$$
\text { Ho. }
$$



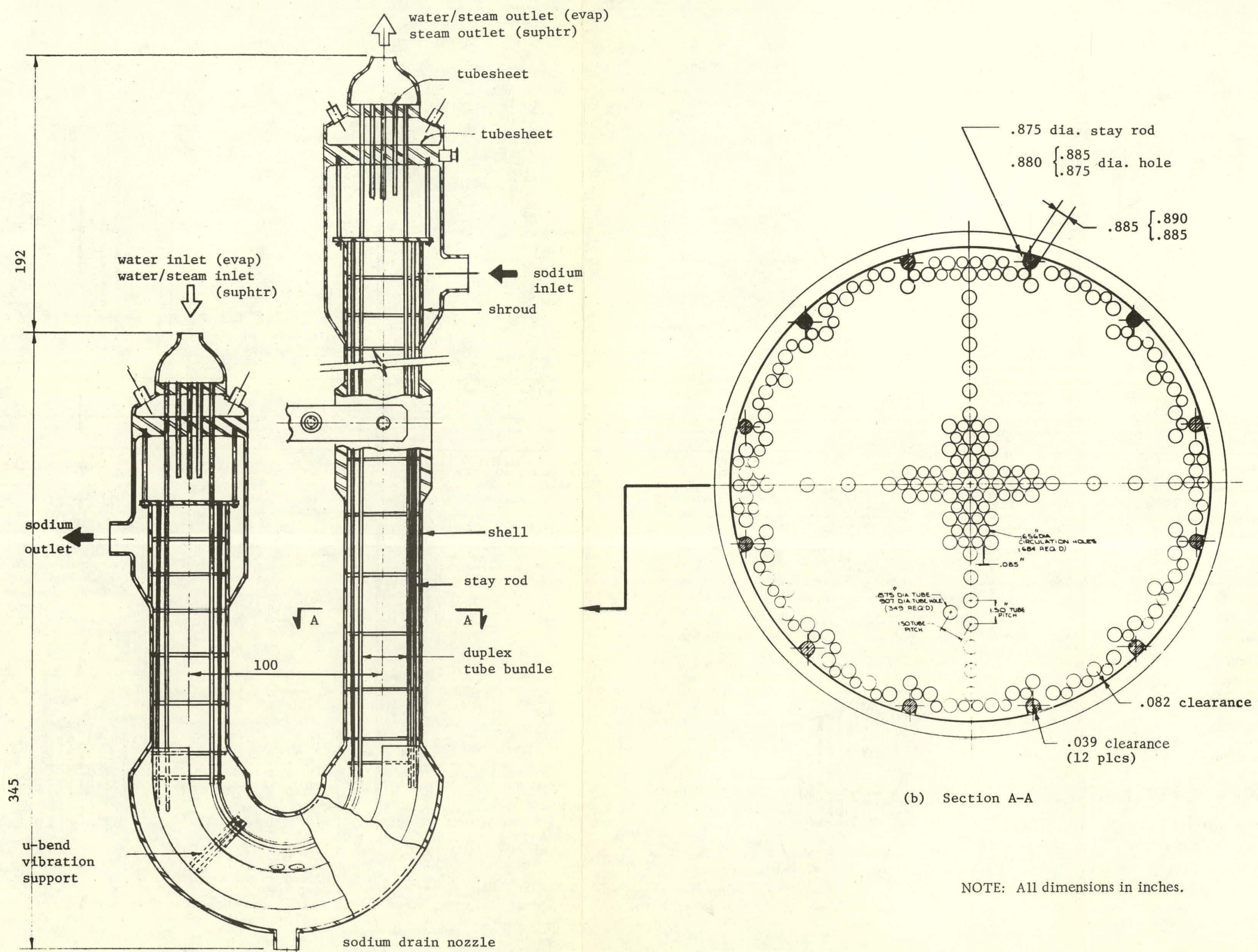

(a) Evaporator and Superheater

(b) Section A-A

NOTE: All dimensions in inches.

Fig. 4. Duplex J-tube Concept: W Steam Generator. (Used with permission of Westinghouse-Tampa Division.) 


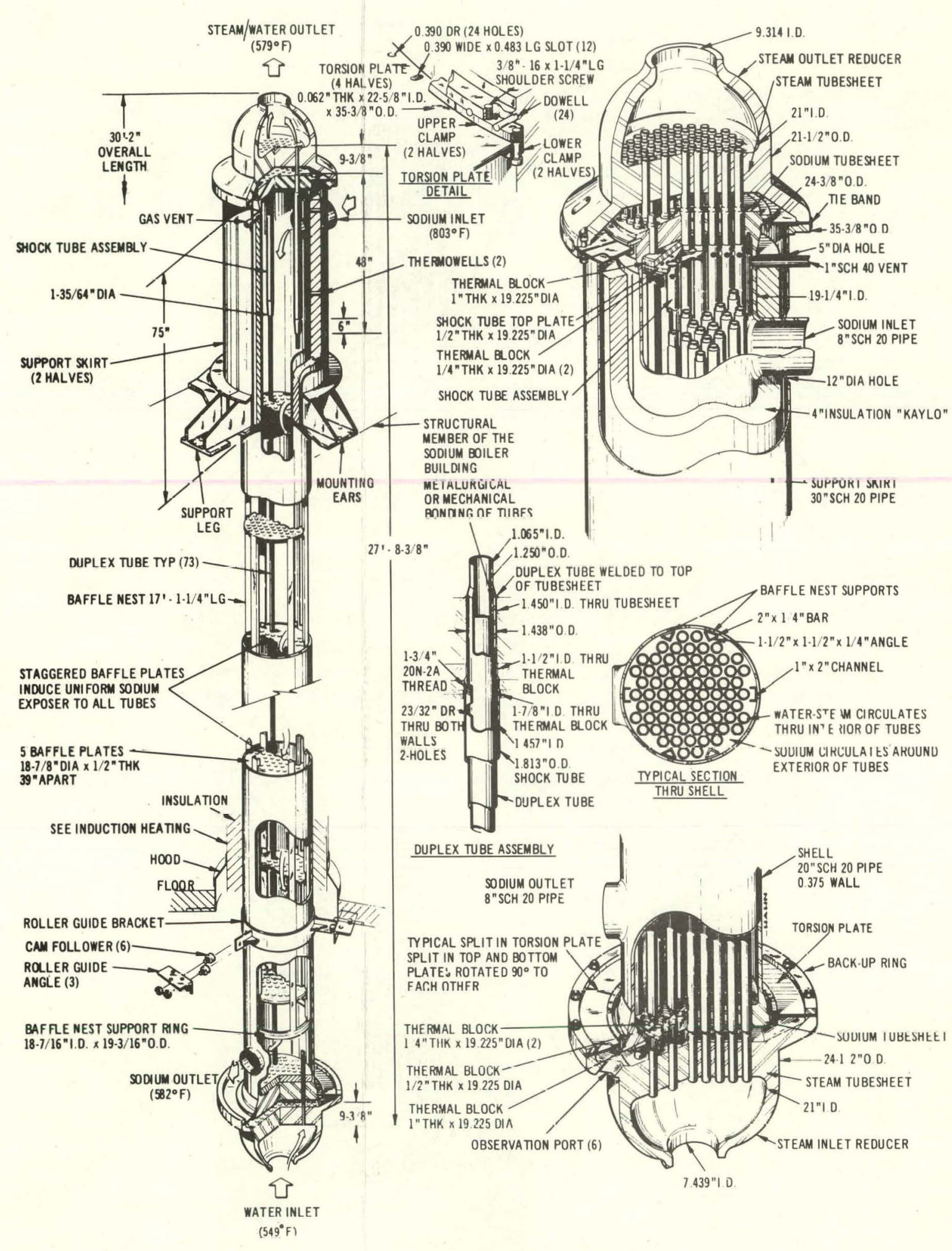

(a) Evaporator

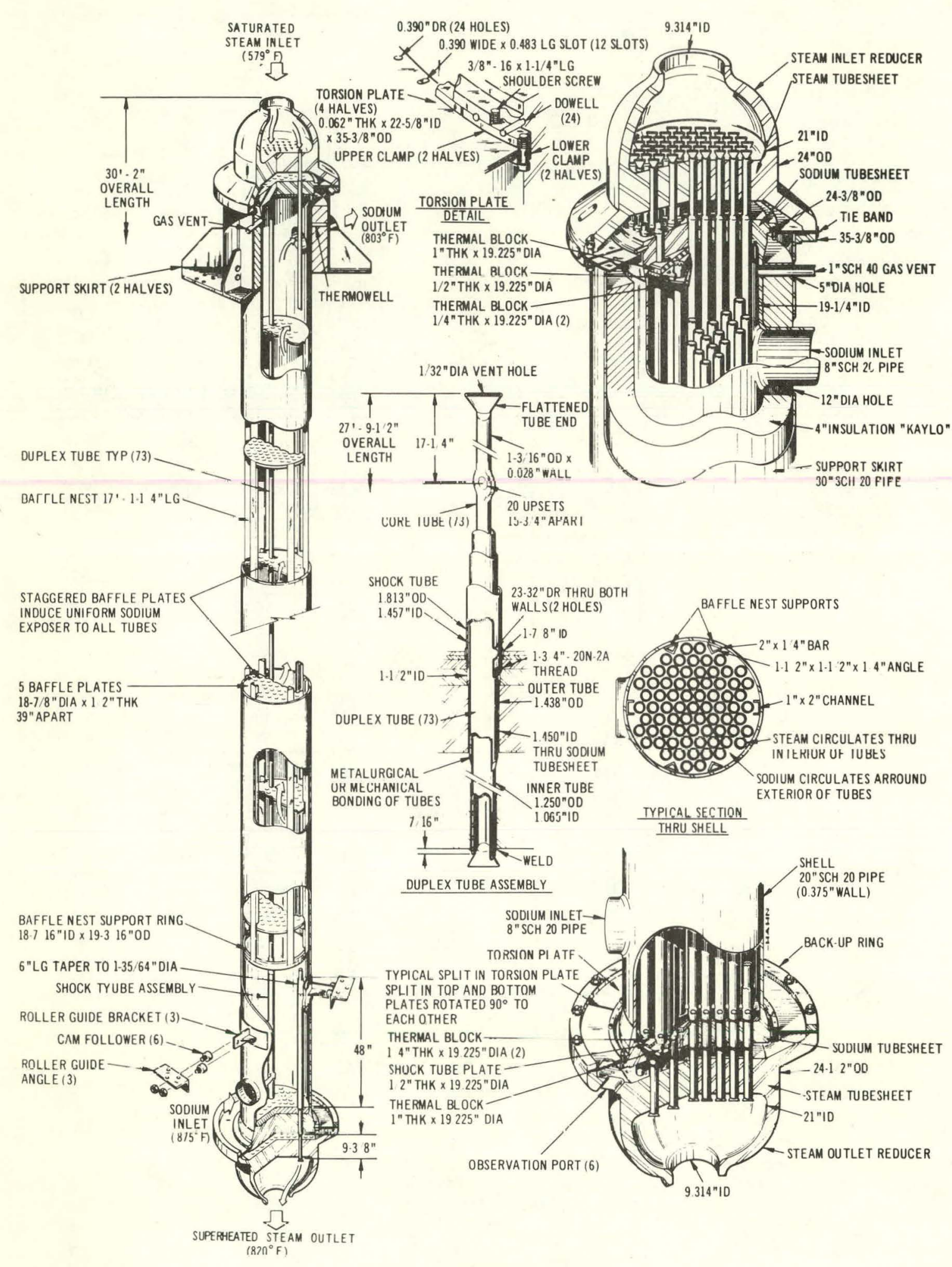

(b) Superheater

Fig. 5. EBR-II Steam Generator (ANL) 
against thermal shock and loading caused by impingement of hot sodium. The shell encloses the entire internals. The potential for tube vibration in EBR-II steam generator has been evaluated using state-of-the-art technology. ${ }^{16}$

a. Evaporator. Water enters the inlet plenum and flows up through the duplex tubes. Sodium enters the inlet nozzle and flows primarily around the baffles, inducing crossflow over the tubes, with some leakage through the tube/baffle-hole clearances.

b. Superheater. Saturated steam enters the inlet plenum and flows down through the duplex tubes. Sodium enters the inlet nozzle and flows up around the baffles, with some leakage through the tube/baffle-hole clearances.

\section{Enrico Fermi Atomic Power Plant Steam Generator (Figs. 6 and 7)}

This unit was developed by Griscom-Russel Company, built in Laguna Beach, Michigan, and first operated in 1962. There are three steam generators in the plant with one experiencing extensive tube damage due to vibration, ${ }^{12,17}$ as discussed in Sec. III. This unit has U-bent single-walled tubes. Each tube makes 14 horizontal runs from the thermal shield to the tubesheet. Eight tubes are fastened together to form a clip, and each clip of tubes is formed into an evolute curve. The shell encloses the entire internals. Water enters a single nozzle in the upper ring header, flows down the downcomer tubes, and is preheated, evaporated, and superheated as it rises in the serpentine tubes. The superheated steam flows through the ring-steam collection header near the top of the unit and leaves through a single nozzle. Sodium enters the shell through two diametrically opposed inlet nozzles near the top, flows down through the shell, inducing crossflow over the tubes, and leaves through the outlet nozzle in the bottom head. This unit does not have separate evaporators and superheaters.

\section{B. Design Considerations}

The technical design, installation, operation and maintenance of steam generators are described in existing standards and codes, including RRD Standard RDT E4-16T, ${ }^{18}$ TEMA Standards, ${ }^{19}$ and ASME Boiler and Pressure Code. ${ }^{20}$ These are intended to produce a satisfactory design from the standpoint of reliability, safety, and effective heat transfer. However, if the design guidance found in the standards and codes is followed, recent experiences of tube failures from flow-induced vibration, corrosion, wear, and fatigue ${ }^{10-12}$ imply that the current standards may be inadequate and that further research and development are needed to prevent such failures in the future.

Some of the important design parameters affecting flow-induced vibrations are considered below.

\section{Baffle (Tule Suppurt) Spacing}

To raise the fundamental natural frequency of tube spans exposed to crossflow and, thereby, to separate the vortex-shedding frequency from the 

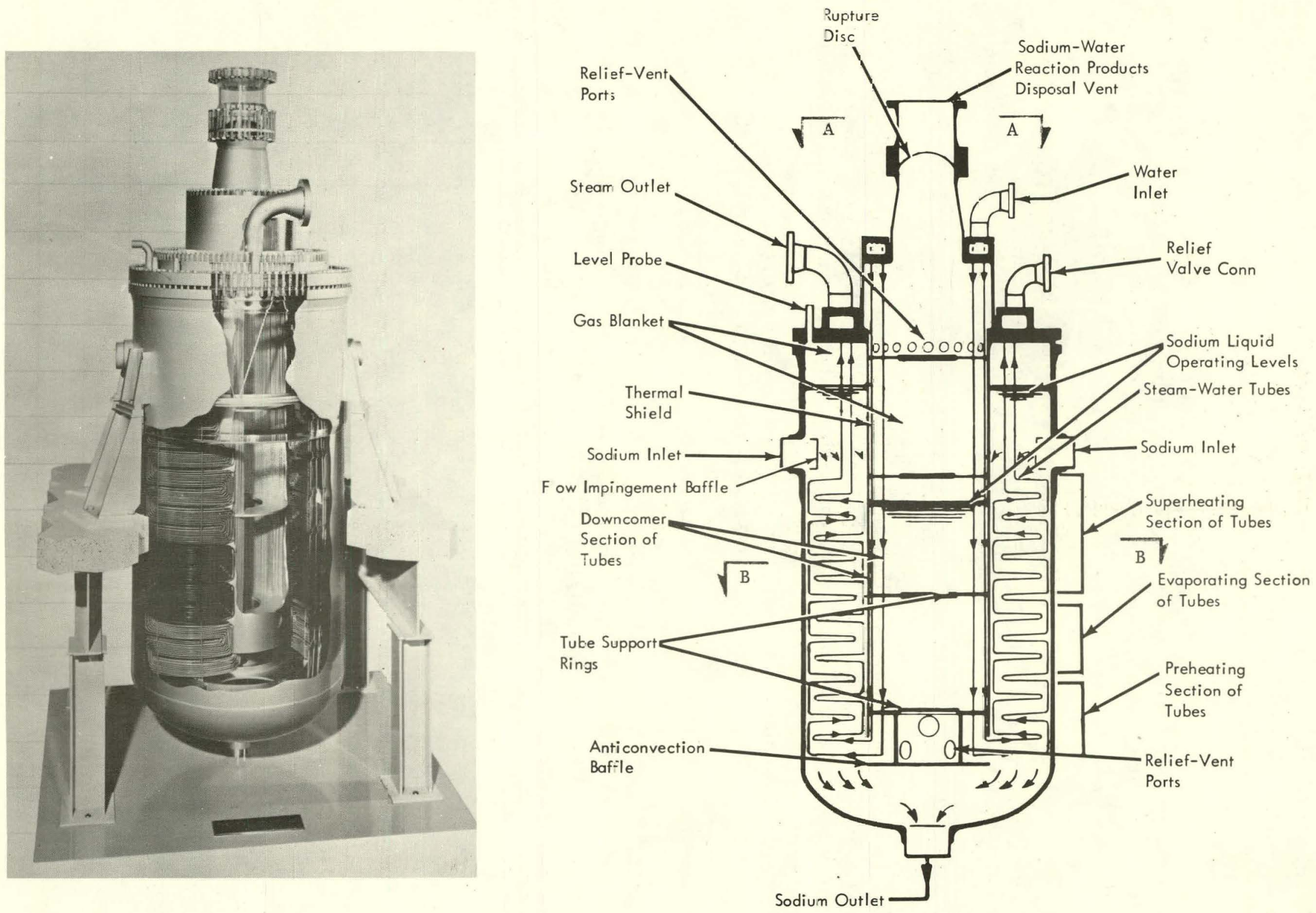

Fig. 6. Errico Fermi Atomic ?ower Plant (EFAPP) Steam Generator. (From Ref. 22.) 


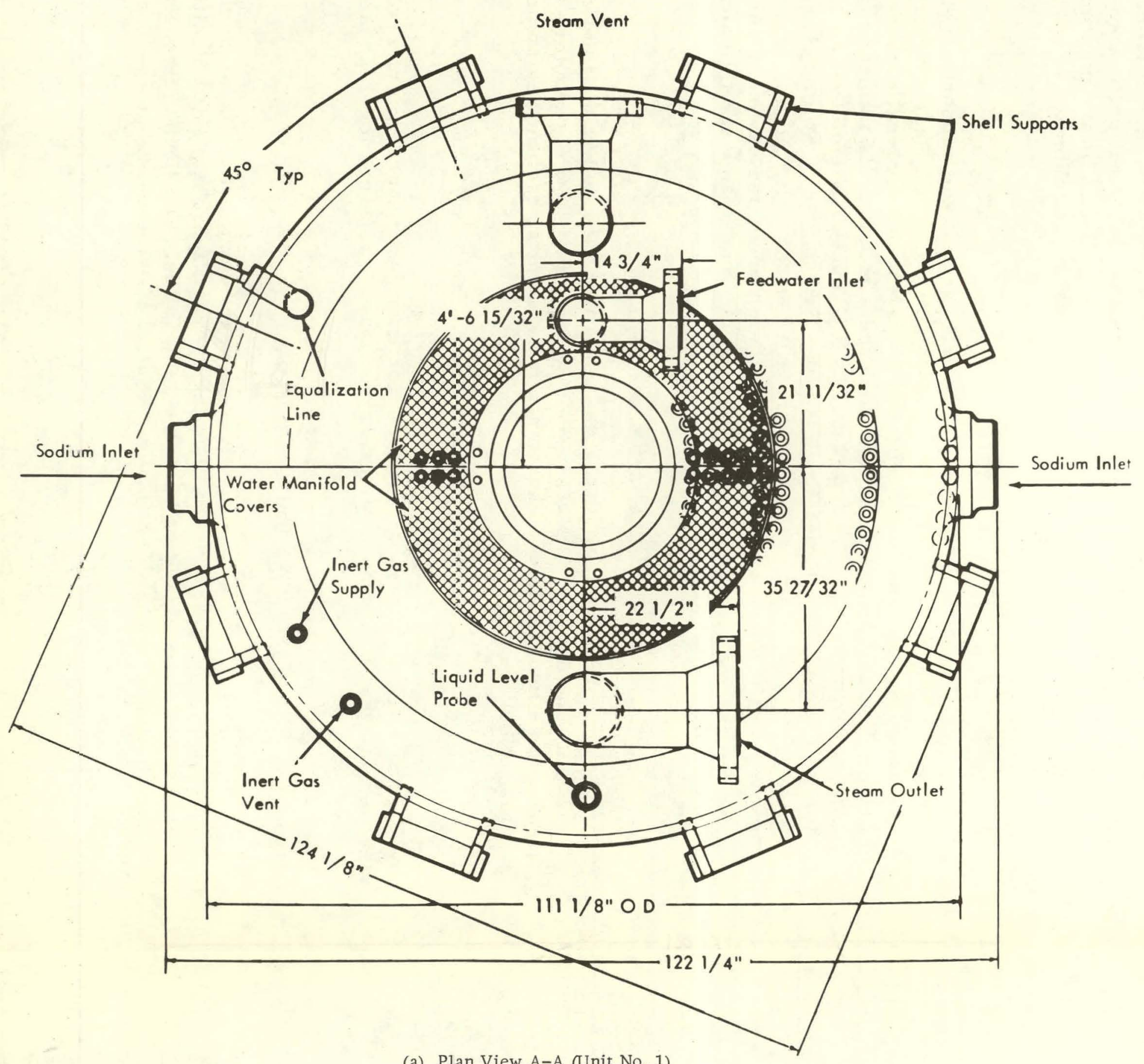

(a) Plan View A-A (Unit No. 1) (See Fig. 6)
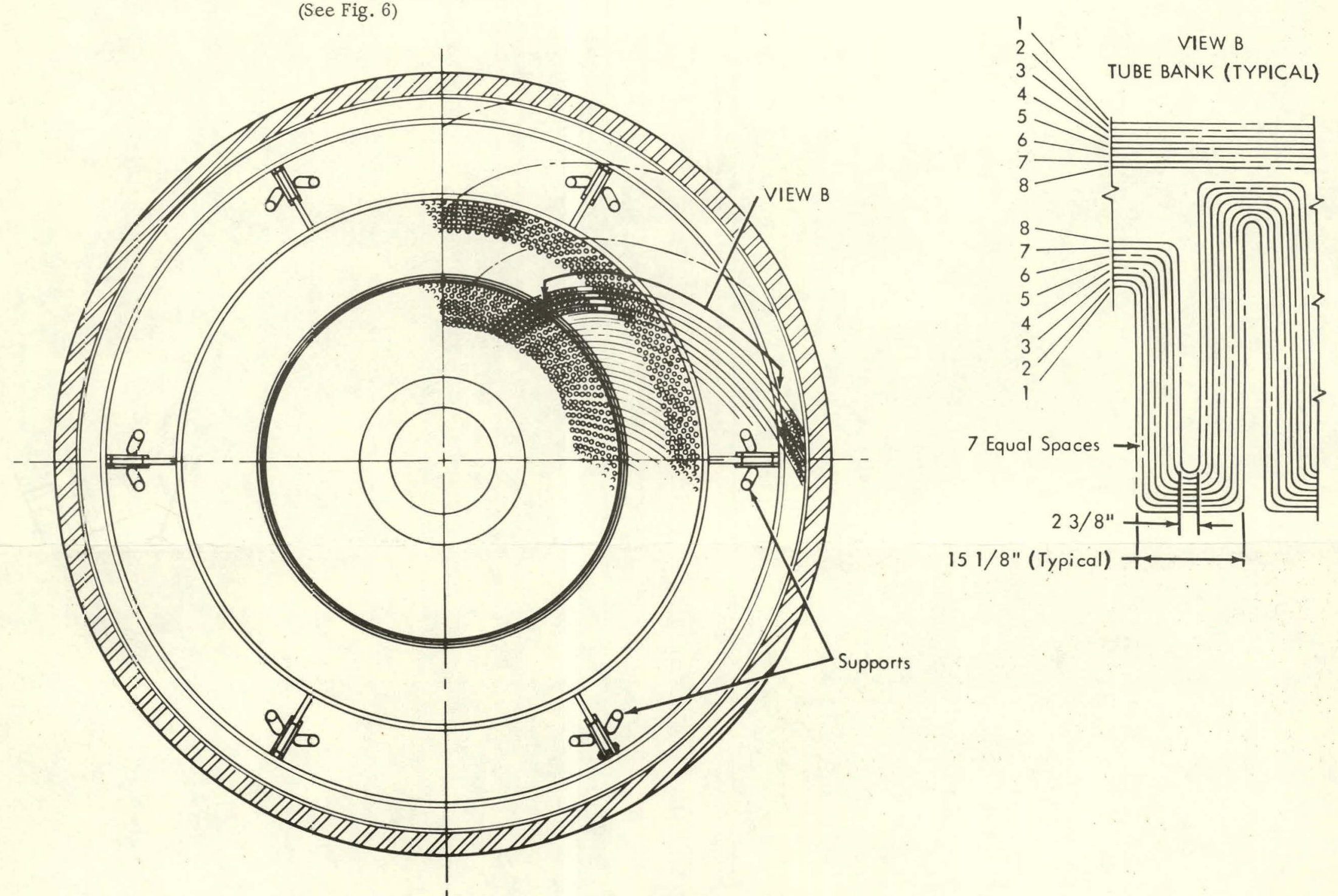

(b) Section B-B (Tube and Tubesheet Arrangement) (See Fig. 6)

Fig. 7. Plan View and Cross Section of EFAPP Steam Generator. (From Ref. 12.) 
tube natural frequencies, multiple supports with a relatively shorter span are used where the predominant fluid-dynamic forces act. For a given number of supports, this technique is effective only if the tubes respond as "single-span" beams between supports, since the lowest natural frequency of a beam on multiple supports will be less than the natural frequency corresponding to the "short-span" length. Consequently, if the tube responds as a beam on multiple supports, the lowest natural frequency may coincide with the frequency at which vortices are shed from the short span, giving rise to a resonant condition. Although increasing the number of baffles or supports is helpful from the standpoint of minimizing the potential for flow-induced vibration, it also increases the pressure drop. To keep the pressure drop low, a minimum number of baffles is desired, and this poses an optimization problem.

\section{Tube-baffle-hole Clearance}

Tube-baffle clearance directly affects the support conditions and influences the vibrational charactcristics of the tube. Experimental results ${ }^{2.1}$ show that dn incredse in lube-baffle-hole clearance generally causes a decrease in natural frequency; this could be a result of its influence on the "effective span length" and/or "damping." Tube-baffle clearance also allows for tube impacting. Experience with the Enrico Fermi Atomic Power Plant Steam Generator ${ }^{12}$ and a turbinc-steam reheater ${ }^{22}$ showed that tube vibration caused baffle-hole enlargement due to hammering and sawing of the tubes in the baffle plates. As a result of this action, tubes can wear thin and partially or completely break off.

\section{Flow Distributor/Impingement Plate}

Impingement plates are generally provided at the inlet regions to prevent tube damage due to severe flow impact. Flow distributors are also used to deflect the flow circumferentially at the crossflow region and to reduce flow velocities.

\section{Vibration Suppressor}

Vibration suppressors, for example, in the form of spacer bars, are placed in bent-tube regions to eliminate low-frequency, high-amplitude response.

\section{Sizing Inlet/Outlet}

For pressure consideration, the inlet/outlet sizes should be as small as allowable, but to reduce the average approach/exit velocities to the tubes, the sizes should be as large as possible. The bound of sensitivity of the above factors should be evaluated before sizing inlet/outlet. 
6. Baffle Thickness, Baffle-hole Shape

Experimental results ${ }^{21,23}$ show that an increase in baffle thickness reduces the fundamental natural frequency. However, the effects of baffle-hole shape are still largely unknown.

\section{Tube Spacing}

The closer the tube spacing in the flow direction in a crossflow region, the higher the frequency of the von Karman Vortex Street. ${ }^{24}$ Vortex shedding is dependent on tube spacing for tube pitch-to-diameter ratios less than two to three times the tube diameter; this range includes typical steam generator tube spacing. Vortex-shedding is discussed in Sec. IV. A.

8. Differential Thermal Expansion

Differential thermal expansion between the shell and tubes can induce axial loads in the tube, causing a change in tube natural frequency; a compressive load reduces tube frequency. The effect of axial force on tube frequency is discussed in Sec. V.C. To minimize the effect of differential thermal expansion, cold-springing techniques were used in the fabrication of the EBR-II steam generators and evaporators. ${ }^{16}$ Flexible bellows in the shell structure also have been used, but are generally not accepted today. The CRBRP candidate designs allow for this phenomenon by providing "bent-tube" regions to accommodate axial motion or by using tube arrangements that are free to "grow," as in the bayonet-tube concept. ${ }^{9}$

\section{Shell-side Flow Orientation}

Traditionally, heat exchangers have been designed with baffle arrangements to promote crossflow, the idea being that crossflow introduces turbulence and improves heat transfer between the tubes and the fluid. This can be an important consideration when the shell-side fluid is water. However, with liquid sodium on the shell side, the heat-transfer characteristics are such that increased turbulence induced by crossflow is not required, since satisfactory heat transfer is obtained with axial flow. Crossflow has a much higher potential than parallel flow for causing detrimental tube vibration, as discussed in Sec. IV. Consequently, from the standpoint of flow-induced vibration, it is advantageous to design units as primarily parallel-flow units. 


\section{FAILURE EXPERIENCES}

Operating experience $9-12,25$ with nuclear-power-plant steam generators shows that the units have incurred extensive damage due to excessive flowinduced tube vibrations. Tube vibrations, in addition to acting as a primary damage mechanism, can accelerate propagation of small cracks caused by other sources such as corrosion. The most vulnerable locations for damage due to vibrations have been observed at antivibration bar regions (San Onofre), the bend region (KWO), and shell-side inlet regions (San Onofre, Enrico Fermi). The types of tube-vibration damage observed include: tube-wall thinning and baffle-hole enlargement due to tube-to-baffle impacts, and tube cracks due to tube-to-tube collisions. Tube damages have not been reported in parallelflow regions.

Operating experience with the Enrico Fermi Atomic Power Plant (EFAPP) steam generator ${ }^{12}$ dramatically illustrated the extensive tube damage that can be caused by flow-induced vibration. Figure 8 shows tube-wall thinning at the inner support. Figure 9 shows two tubes broken at the support bars; tube failure might be attributed to large-amplitude oul-uf-plane vibration of the unsupported bend. Figure 10 shows large cracks in a tube, caused by collision with adjacent tubes. Figure 11 shows the enlargement of supportbar holes, which may be caused by tubes rubbing in holes. Figure 12 shows samples of the broken tubes that were located as shown in Fig. 11. The peculiar "knife-cut" tube damage was caused by collision with adjacent tubes.

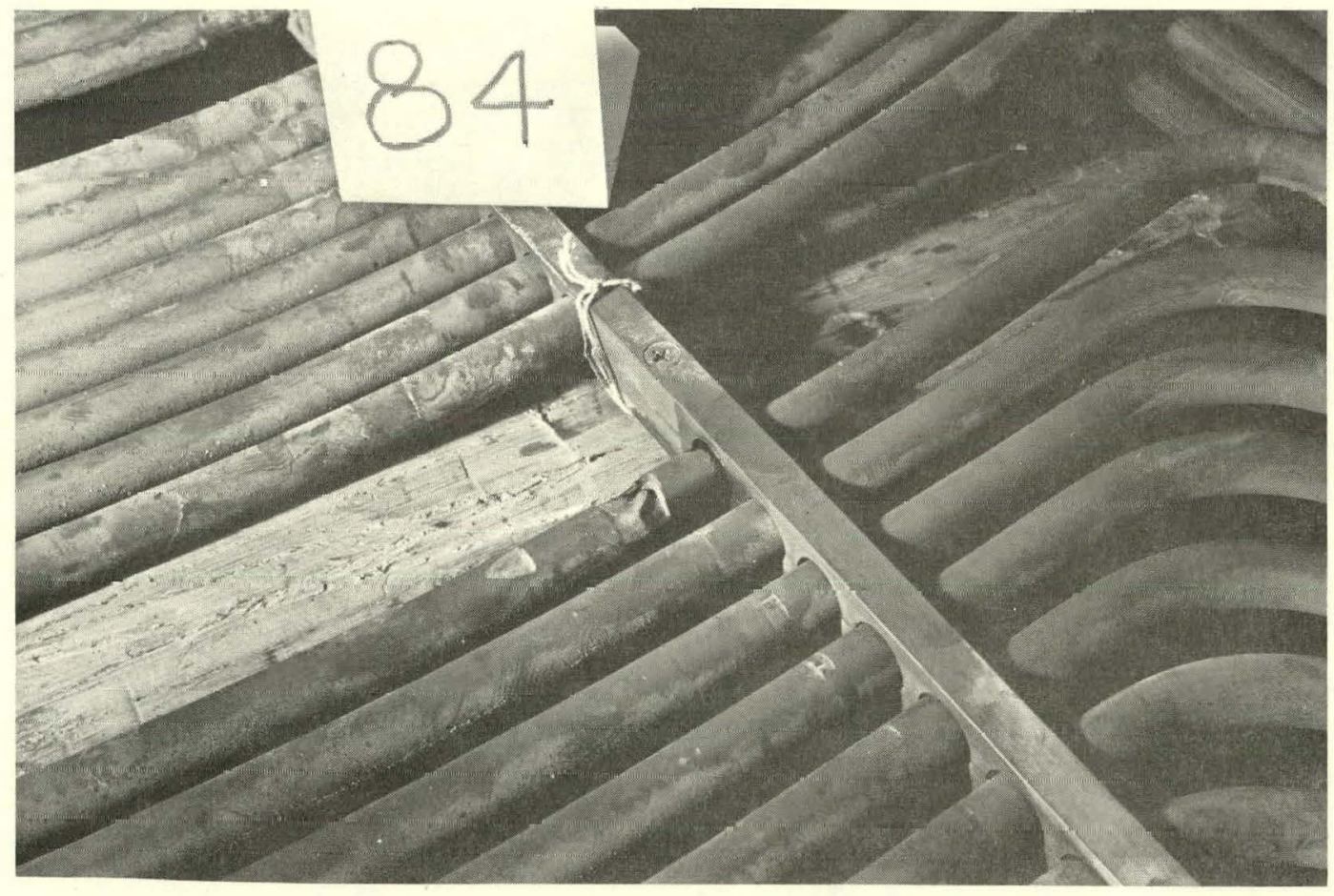

Fig. 8. EFAPP Steam Generator: Tube-wall Thinning Caused by Rubbing Inside of Support Bars. (From Ref. 26.) 


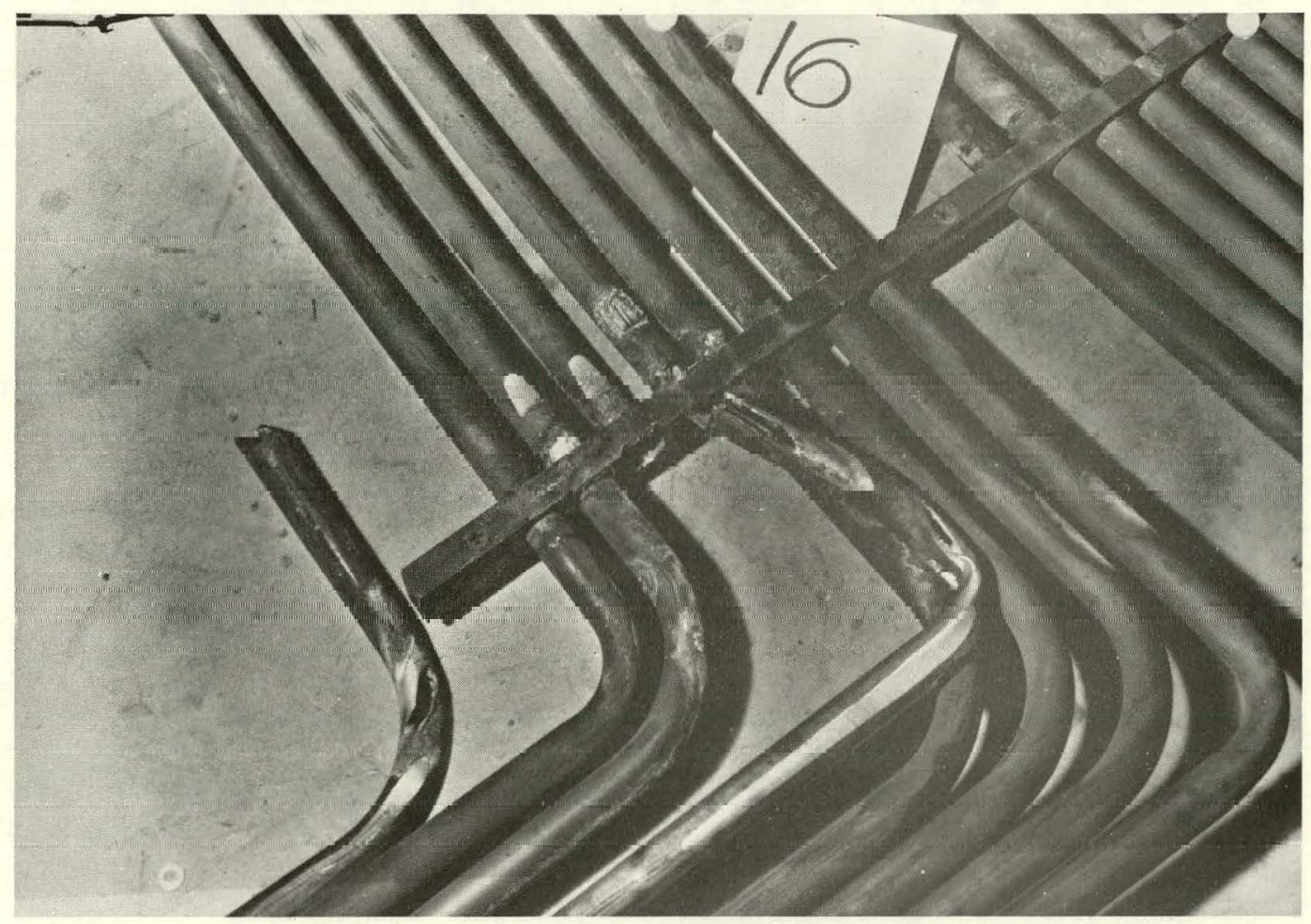

Fig. 9. EFAPP Steam Generator: Broken Tubes at Support Bar due to Excessive Tube Vibration. (From Ref. 26.)

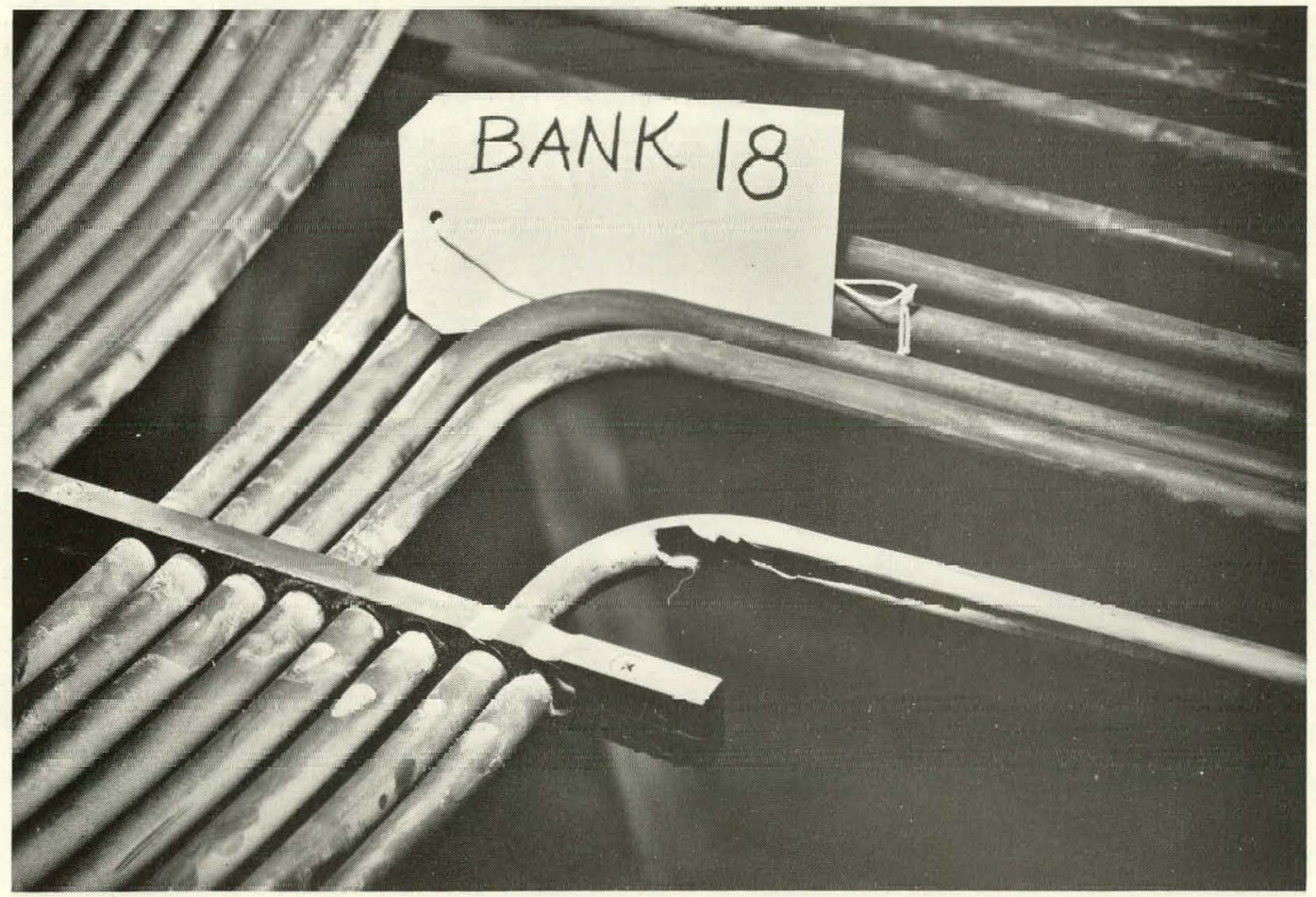

Fig. 10. EFAPP Steam Generator: Tube Crack due to Collision with Adjacent 'l'ubes. (From Ret. 26.) 


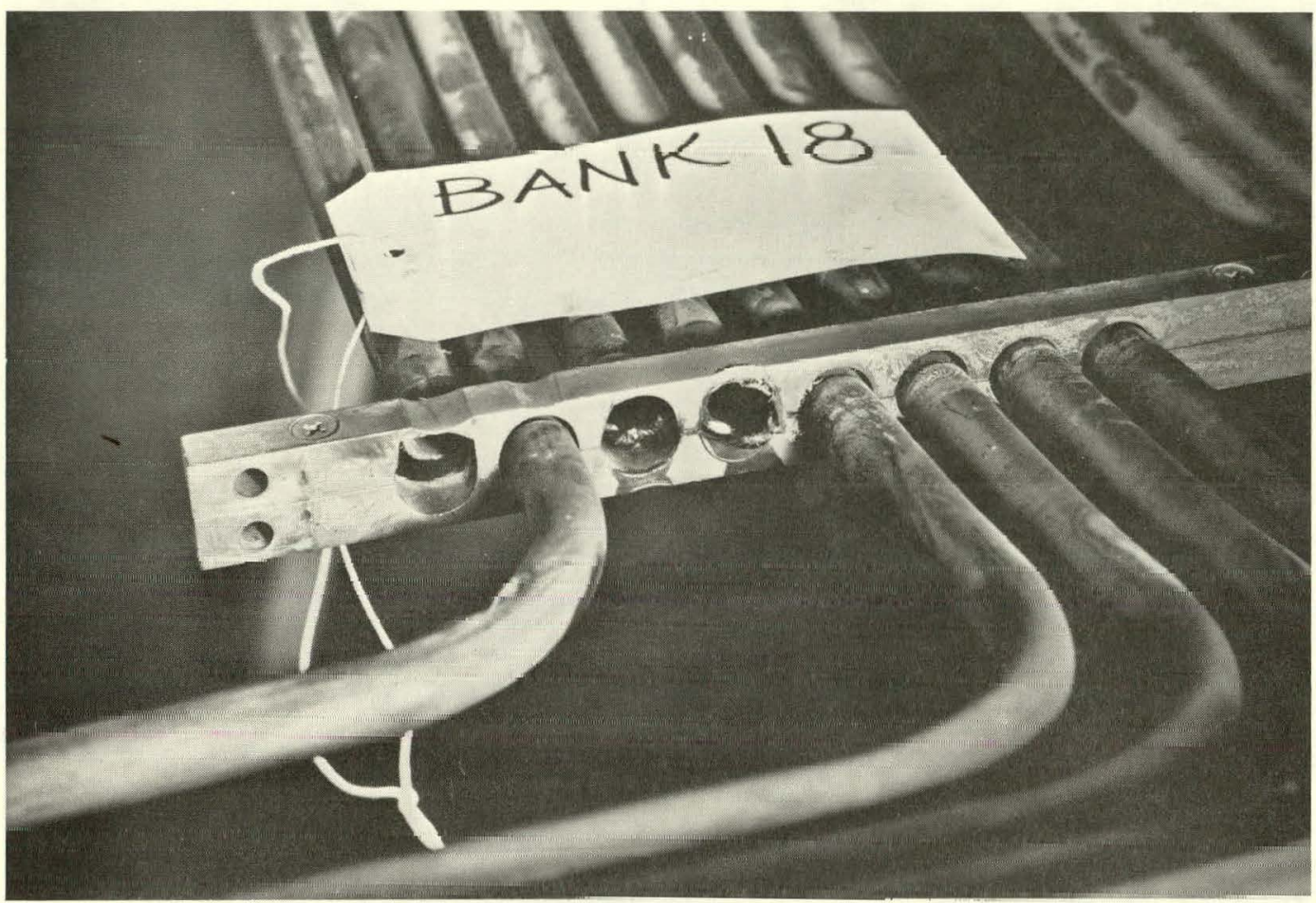

Fig. 11. EFAPP Steam Generator: Enlargement of Support-bar Holes due to Tube-to-Support-bar Impacts. (From Ref. 26.)

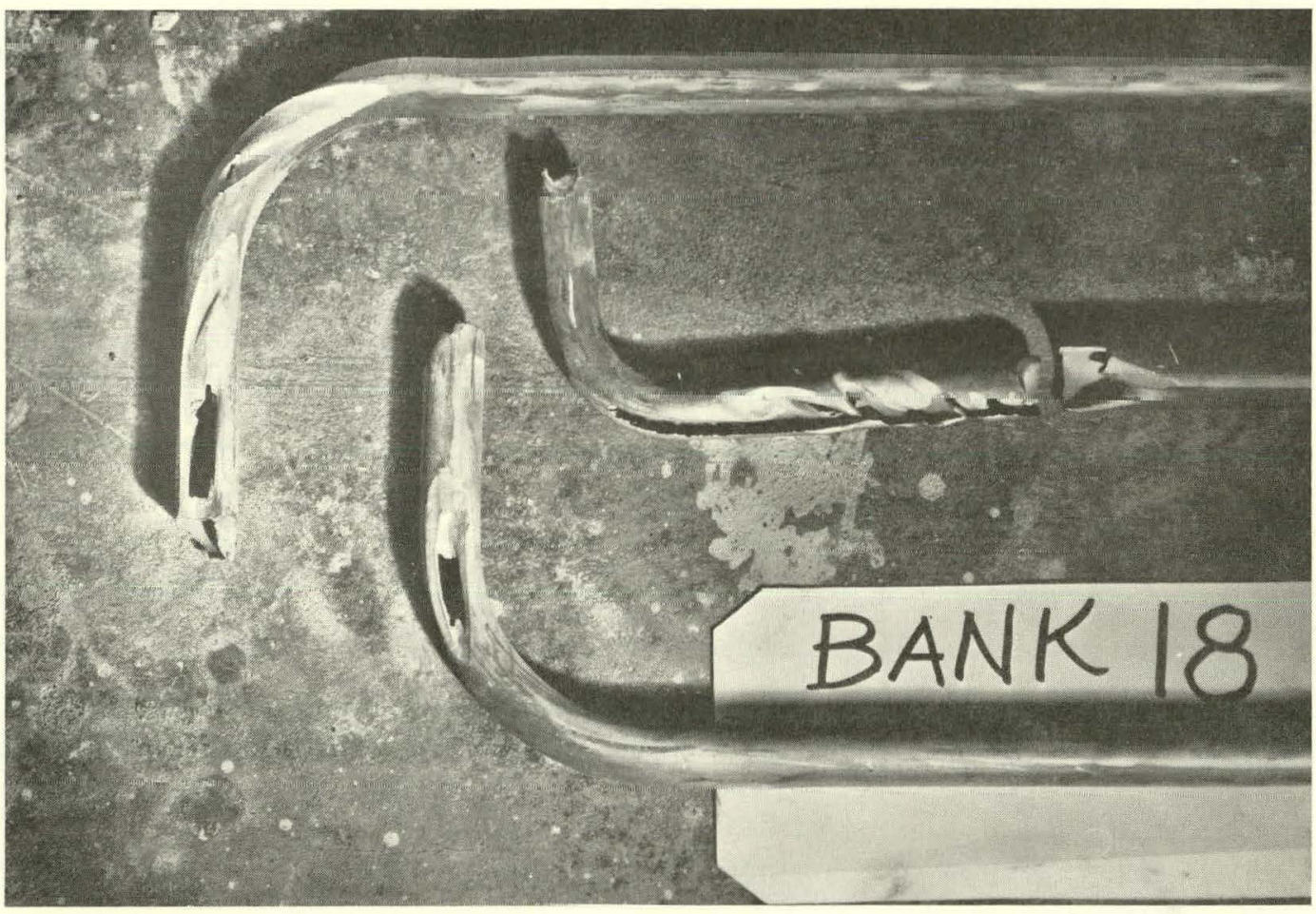

Fig. 12. EFAPP Steam Generator: Samples of Broken Tubes with "Knife-cut" Damage by Tube-to-Tube Collision. (From Ref. 26.) 
Most of the vibration damage occurred at the inlet regions. The general causes of vibration damage were identified as:

1. Insufficient restraint of tubes in the region near the shell-side inlets.

2. Insufficient baffling at the shell-side inlets to reduce the flow velocity to a satisfactory value.

Although not firmly established, the excitation mechanism was most probably either a resonant condition between tube natural frequency and vortexshedding frequency, or a fluidelastic instability.

In addition to the foregoing steam-generator failures, a survey by Nelms and Segaser, ${ }^{1}$ of primary-circuit heat exchangers in USERDA-operated reactors, revealed that nine out of the 17 heat exchangers had experienced some form of vibration-induced tube failure. It is also known that flowinduced tube-vibration failures have plagued the heat-exchanger industry for years. When the shell- and tube-side liquids are both water or water and steam, a small number of tube failures can generally be tolerated, and the failed tubes can be plugged with only a slight decrease in system performance. However, in an LMFBR steam generator, the consequence of a tube failure is much more serious, due to the ensuing sodium-water reaction. 


\section{FLOW-INDUCED EXCITATION MECHANISMS}

Fluid flowing through the shell side of steam generators is a source of energy that can induce and sustain vibrations of the tubes,* baffle/supports, shroud, and shell. Although this report focuses on beamlike vibrations of tubes, many of the basic phenomena and methods of analysis discussed also have application to the design evaluation of other components. Operating experiences show that tube-vibration failures are among the more prevalent of steam-generator mechanical-component vibration failures. Vibrations induced by tube-side flow are usually negligible, except at velocities much higher than those existing in steam-generator tubes; consequently, the shellside flow represents the primary excitation source. Many investigations have been reported concerning flow-induced excitation mechanisms and related vibration responses of a single tube immersed in an "infinite" flowing fluid medium. However, because of the complexities of the flow fields, progress has been slow in developing an understanding of many basic excitation mechanioms.

Flow-induced excitation forces on steam-generator tubes and other internal components can arise from several mechanisms:

1. Vortex shedding.

2. Turbulent buffeting.

3. Fluidelastic whirling.

4. Jet switching.

5. Turbulent pressure fluctuations associated in parallel flow.

6. Hydraulic noise.

7. Structural-borne noise: vibration transmitted through piping, supports, and external mechanical vibration transmitted to the units,

8. Oscillations or pulsations in the flow.

The last two excitation mechanisms are system-dependent and will not be discussed here.

A. Vortex Shedding

In two-dimensional fluid flow across a single cylinder or arrays of parallel cylinders, vortices are formed in the wake behind the cylinder. The nature of the vortex shedding is dependent on the Reynolds number.

*Circular tubes, unless denoted otherwise. 


\section{Reynolds Number}

The Reynolds number, one of the most important dimensionless numbers in characterizing fluid-flow regimes, represents the ratio of the fluid inertia force to the frictional force. It is directly proportional to the flow velocity and is expressed in the form

$$
\operatorname{Re}=\frac{\rho V d}{\mu} \text { or } \frac{V d}{\nu},
$$

where

$$
\begin{aligned}
\operatorname{Re} & =\text { Reynolds number } \\
\rho & =\text { mass density of fluid, } \\
\mu & =\text { viscosity of fluid, } \\
\nu & =\text { kinematic viscosity of fluid, } \\
\mathrm{V} & =\text { free-stream velocity }
\end{aligned}
$$

and

$$
\mathrm{d}=\text { outer diameter of cylinder.* }
$$

Dynamic similarity of two flow fields requires, among other things, that the Reynolds numbers be equal for both. The viscosity of liquids $(\mu)$ is nearly independent of pressure and decreases at a high rate with increasing temperature. The kinematic viscosity of liquids behaves like $\mu$, since the density of liquids changes only slightly with pressure and temperature. ${ }^{27}$ The Reynolds number in LMFBR steam generators is generally in the range $10^{3}-10^{5}$.

The major regimes of fluid flow across a rigid circular cylinder are shown in Fig. 13. Marris ${ }^{3}$ explained the development of wakes behind the cylinder for different Reynolds-number regimes on the basis of earlier work by Föppl, ${ }^{28}$ Roshko, ${ }^{29,30}$ Tritton, ${ }^{31}$ Kovasznay, ${ }^{32}$ Birkhoff, ${ }^{33,34}$ Humphreys, ${ }^{35}$ Schaeffer and Eskinazi, ${ }^{36}$ and others, as follows.

At extremely low Reynolds numbers $(<5)$, the flow streamlines close behind the cylinder and the flow does not separate, as shown in Fig. 13a. However, as the Reynolds number is increased, the streamlines widen, and at a Reynolds number of 5-15, a pair of fixed "Föppl" vortices first appears immediately behind the cylinder, as shown in Fig. 13b. Kovasznay and Tritton showed and confirmed that the vortex pair behind the wake of cylinders becomes unstable at a Reynolds number of about 40 , and the fixed vortex pair separates from the main body of the fluid. At a Reynolds number of about 90 , one of the fixed vortices breaks away from the cylinder. This causes a wakepressure asymmetry and the other leaves, the process repeats itself, and the

*Circular cylinder unless denoted otherwise. 
state of alternating vortex shedding is attained, as shown in Fig. 13c. Roshko's observation showed that for Reynolds numbers less than 150 , for which the free vortex layers roll up into vortices prior to transition, the resulting vortices are purely viscous. The fluid in such vortices is laminar, and the vortex street is preserved for many diameters downstream. As the Reynolds number is increased beyond the vortex-shedding point to 150-300, a laminarto-turbulent transition begins in the free vortex layers before breaking away into the street, as shown in Fig. 13d. At a Reynolds number of about 300, and continuing up to $\sim 3 \times 10^{5}$, the vortex street is fully turbulent. In the Reynoldsnumber range $3 \times 10^{5}$ to $3.5 \times 10^{6}$, the laminar-boundary layer has undergone turbulent transition, the wake is narrower and disorganized, and no vortex street is apparent, as shown in Fig. 13e. As the Reynolds number is increased beyond $3.5 \times 10^{6}$, the turbulent vortex street forms and the wake is thinner, as shown in Fig. $13 f$.

(a)

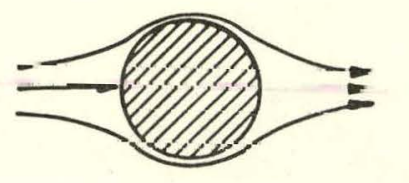

Re 5 RFGIMF OH UNSEPARATEO FLOW.

(b)

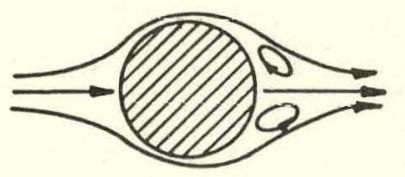

5 TO $15 \leqslant R Q<40$ A FIXED PAIR OF FOPPL VORTICES IN THE WAKE.

(c)

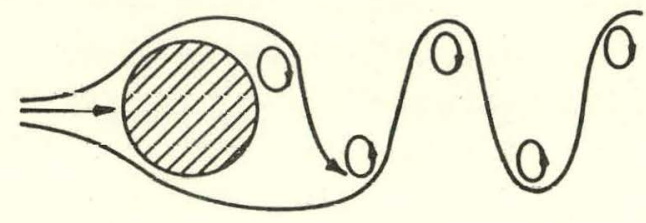

$40 \leqslant R_{e}<90$ AND $90 \leqslant R_{e}<150$ TWO REGIMES IN WHICH VORTEX STREET IS LAMINAR:

PERIODICITY GUVERINEO IN LOW

Re RANGE BY WAKE INSTABILITY

PERIODICITY GOVERNED IN HIGH

Re RANGE BY VUKIEX SHEDDING

(d)

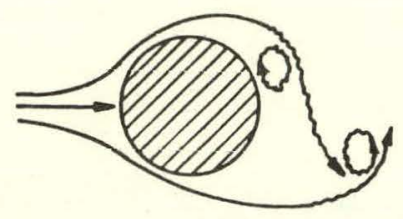

$150<R_{\theta}<300$

TRANSITION RANGE TO TURBULENCE IN VORTEX.

$300 \leqslant$ Re $₹ 3 \times 10^{\circ}$ VORTEX STREET IS FULLY TURBULENT.

(e)

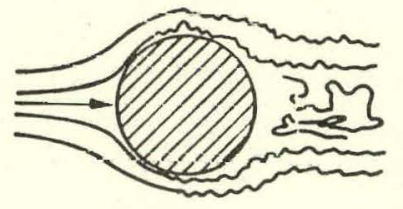

$3 \times 10^{8} \prec R_{e}<3.5 \times 10^{\circ}$

LAMINAR BOUNDARY LAYER HAS UNDERGONE TURBULENT TRANSITION. THE WAKE IS NARROWER AND DISORGANIZED.
VORTEX STREET IS APPARENT.

$3.5 \times 10^{\circ}<\mathrm{Re}<\infty$ (?)

RE-ESTABLISHMENT OF THE TURBU LENT VORTEX STREET THAT WAS

THIS TIME THE BOUNDARY LAYER

IS TURBULENT AND THE WAKE

IS THINNER.

Fig. 13. Regimes of Fluid Flow across Rigid Circular Cylinder. (From Ref. 4.) 


\section{Strouhal Number}

The Strouhal number (sometimes called reduced frequency) is another important dimensionless number which characterizes the frequency at which vortices are shedded from a rigid circular cylinder; it is defined by

$$
S_{\mathrm{v}}=\frac{f_{\mathrm{v}} \mathrm{d}}{\mathrm{V}}
$$

where

$$
\begin{aligned}
S_{\mathrm{v}} & =\text { Strouhal number } \\
\mathrm{f}_{\mathrm{v}} & =\text { vortex-shedding frequency (vortex pair), } \\
\mathrm{d} & =\text { outer diameter of cylinder }
\end{aligned}
$$

and

$$
\mathrm{V}=\text { free-stream velocity. }
$$

The Strouhal number depends on the Reynolds number. Lienhard ${ }^{4}$ and Chen ${ }^{24,37}$ reviewed experimentally determined data on the StrouhalReynolds-number relationship for flow crossing an isolated single stationary

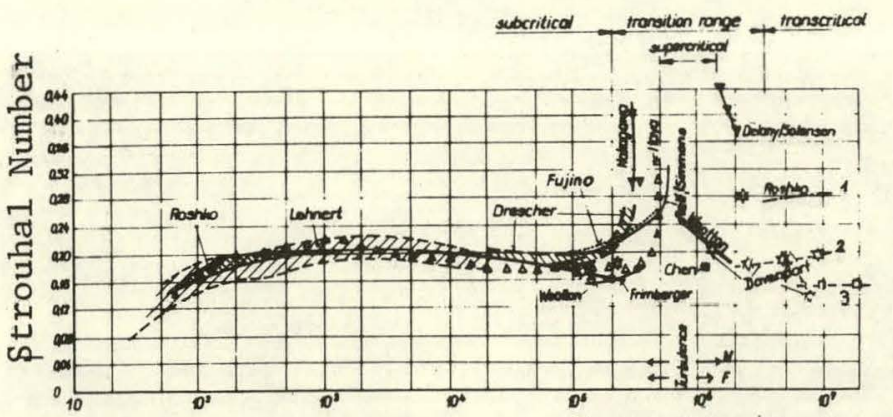

zoziz Compiled by Letmert (1937) Reynolds Number

$=505$ Drescher (1956)

Fujino (1957)

-... (Roshko (1961)

- Average line alker Delony and Sokensen (1953)

+ Noumon und Fioffer (858)

- Obsenation by the authour an a metal shck (henht $90 \mathrm{~m}$, dianeter $22 \mathrm{~m}$ )

- Wootton ( 1968$)$ mith rough cylinder $\left(\right.$ Wd . $\left.013 \times 10^{-3}\right)$

- - 2 - - Absenvation on metal slacks comaled by Daver wuri (1959)

- - a - abservation on conc rete stocks compiled by Davenport (1959)

- Frmberyer (105\%)

Roshiko (9956)

$\longrightarrow$ N investigated fiemalds number ronge by Novogawo ef al (1505)! Jurbolence

$\longleftrightarrow$ F investigated Reynolds number rorge by fung (1060) Sormat f rigion

$\Delta \Delta \Delta \Delta$ itoya/Yasuda (1961), anly the overage in e, with slationary cyinder

$\rightarrow-a$ Wotton (1968) with sma.th i cyinder

$\rightarrow-$ Nokogana (1963)etal

Riesults of UTIA (1955) for R.0.6 10 2.10 fils to the upper curve group (ollier Heele 1961)

Fig. 14. Experimentally Determined StrouhalReynolds-number Relationslıip for Ciscular Cylinders. (From Ref. 37.) cylinder, and plotted the results as shown in Fig. 14. The Strouhal number remains nearly constant with a value of 0.21 within the range of Reynolds numbers from 300 to about $2 \times 10^{5}$, defined as the subcritical range. As the Reynolds number is further increased to about $3.5 \times 10^{5}$, the Strouhal number seems to increase due to the narrowing of the wake of the flow. Beyond a Reynolds number of about $3.5 \mathrm{x}$ $10^{6}$, the Strouhal number again seems to remain constant with $\mathrm{S}_{\mathrm{V}} \cong 0.27$ as the turbulent vortex street reestablishes. Lienhard $\mathrm{d}^{4}$ proposed a reasonable envelope within $\pm 5 \%$ accuracy over a large Reynolds-number range, as shown in Fig. 15.

Again, the discussions so far are based on the postulate that two-dimensional uniform flow 


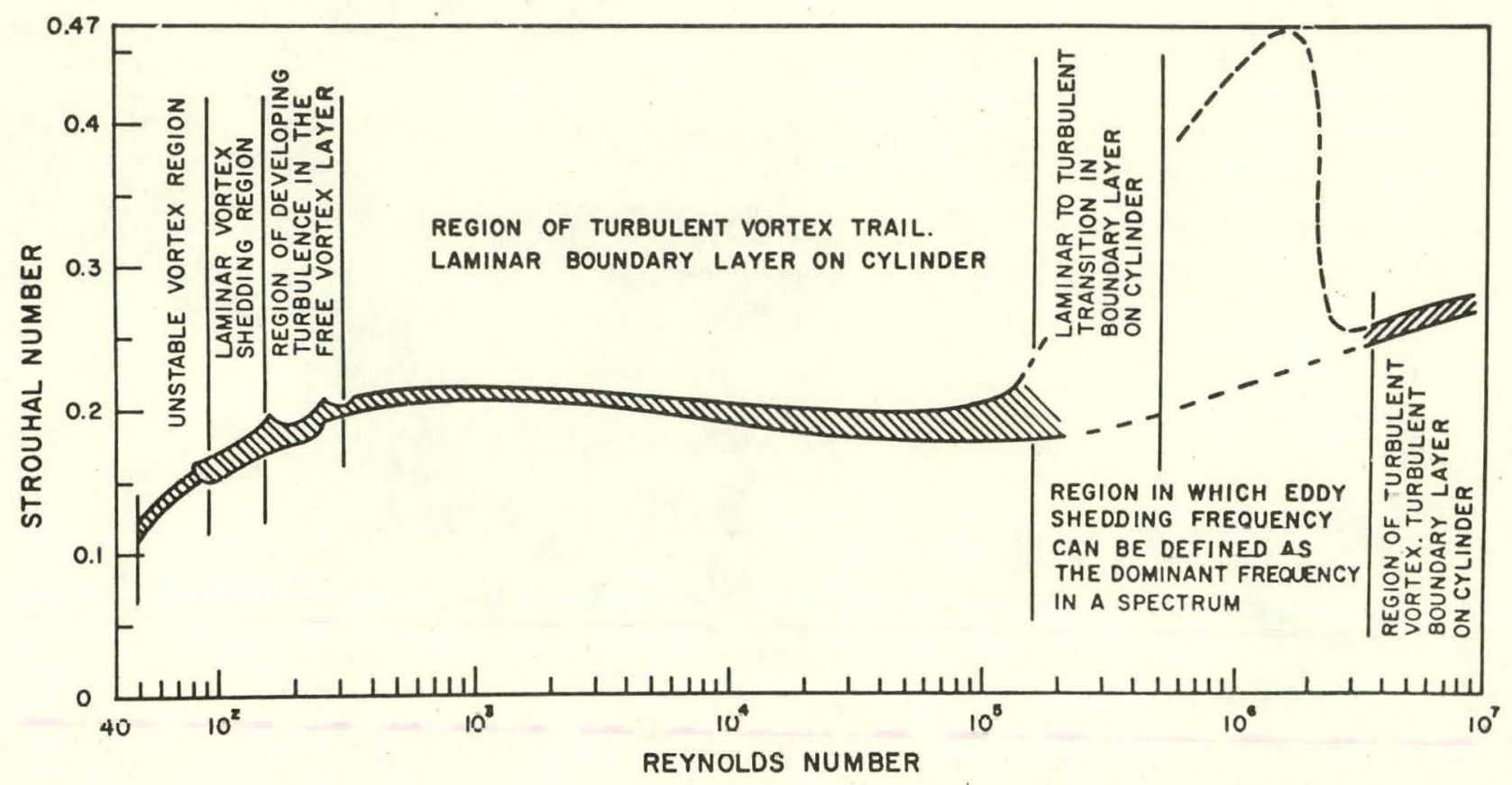

Fig. 15. Envelope of Strouhal-Reynolds-number Relationship for Circular Cylinders. (From Ref. 4.)

crosses a single stationary cylinder whose axis is perpendicular to the flow direction. If flow approaches a cylinder at an angle other than perpendicular to the longitudinal axis of the cylinder, it is intuitively appealing to resolve the flow velocity into cross - and parallel-flow components, and use the crossflow component to compute the vortex-shedding frequency and crossflow response. Unfortunately, there is little information available to evaluate the validity of this approach. In the low-Reynolds-number range, $50 \leq R_{n} \leq 150$, the Strouhal number based on the crosstlow component of velocily was found to bc approxsi mately constant for yaw angles up to $30^{\circ}$; for larger yaw angles, a significant monotonic increase was observed. ${ }^{38}$ For high Reynolds numbers, the effect of yaw angle on Strouhal number has not been established. For stream angles close to $90^{\circ}$, the flow can be considered as passing over an elliptical cylinder. Generally, however, flow patterns in steam generators are not sufficiently defined to allow resolving the flow vector into its cruss-and parallel-flow components. Moreover, the flow characteristics within a tube bundle are much more complicated than for an isolated single tube, with the complexity increasing further for flexible tubes that are vibrating.

\section{Strouhal Number as a Function of Tube Spacing}

At the inlet and outlet zones of steam generators, the shell-side flow is predominantly crossflow with respect to the tube bundle. Unfortunately, only limited results are available on the vortex-shedding phenomena in tube arrays. Chen ${ }^{24}$ showed that the surrounding tubes can have a considerable influence on the values of the Strouhal number. His experimental data are based on gas flow and have not been verified for liquids; nevertheless, his correlations are widely used to evaluate the vortex-shedding frequency/ Strouhal number in steam generators. His results are summarized below. 
Two different tube-array models are considered as shown in Fig. 16; the in-line and staggered tube patterns are typical of present steam generators. The Strouhal numbers obtained in Chen's experiment are functions of the transverse and longitudinal spacing ratios $\mathrm{T} / \mathrm{d}$ and $\mathrm{L} / \mathrm{d}$, respectively, of the tube array. The Strouhal-number/tube-spacing relationships for in-line and staggered tube arrays are shown in Figs. 17 and 18. In general, the Strouhal number increases with decreasing longitudinal spacing ratio. For an in-line tube bank, and a given longitudinal spacing, the Strouhal number increases monotonically with transverse spacing ratio for the range of data reported. For a staggered tube bank and given longitudinal spacing, the Strouhal number peaks at a transverse-spacing ratio of about 2.2; Strouhal numbers as high as 0.7 are reported. For large transverse spacings and small longitudinal spacings, the curves are not complete.

As Nelms and Segaser stated, ${ }^{1}$ if the best estimate of the average crossflow velocity (see Sec. V.A) along the tube span is used in Eq. 2, it seems reasonable that this will yield the best estimate of the average vortex-shedding frequency or predominant frequency, if one exists.

Similar results have been reported by König and Gregorig, ${ }^{39}$ with some discrepancies between their results and those of Chen; however, Chen's results remain widely accepted.

\section{Drag and Lift Forces}

The fluid-dynamic forces exerted by crossflow acting on a cylinder can be rcsolved into two types (see Fig. 19): (1) drag forces $F_{D}$ acting in the direction of flow, and (2) lift forces $F_{L}$ acting in the transverse-to-flow direction.

At Reynolds numbers $\leq 40$, only a steady drag force exists, and because of the symmetry of the flow, the lift force is zero (see Fig. 13b). As the Reynolds number increases above 40 , the drag force remains steady until the vortices become unstable. At that point, a drag force develops that

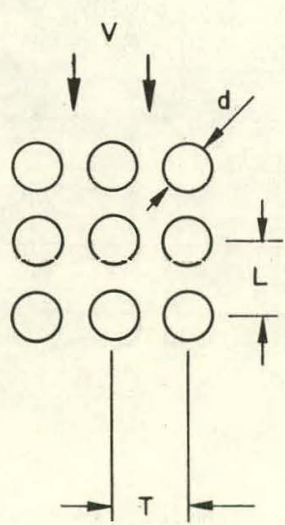

IN-LINE

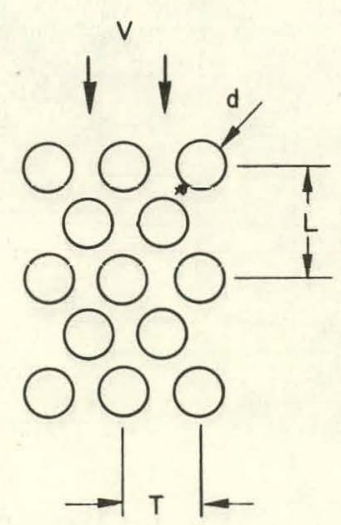

STAGGERED
Fig. 16

Tube Patterns 


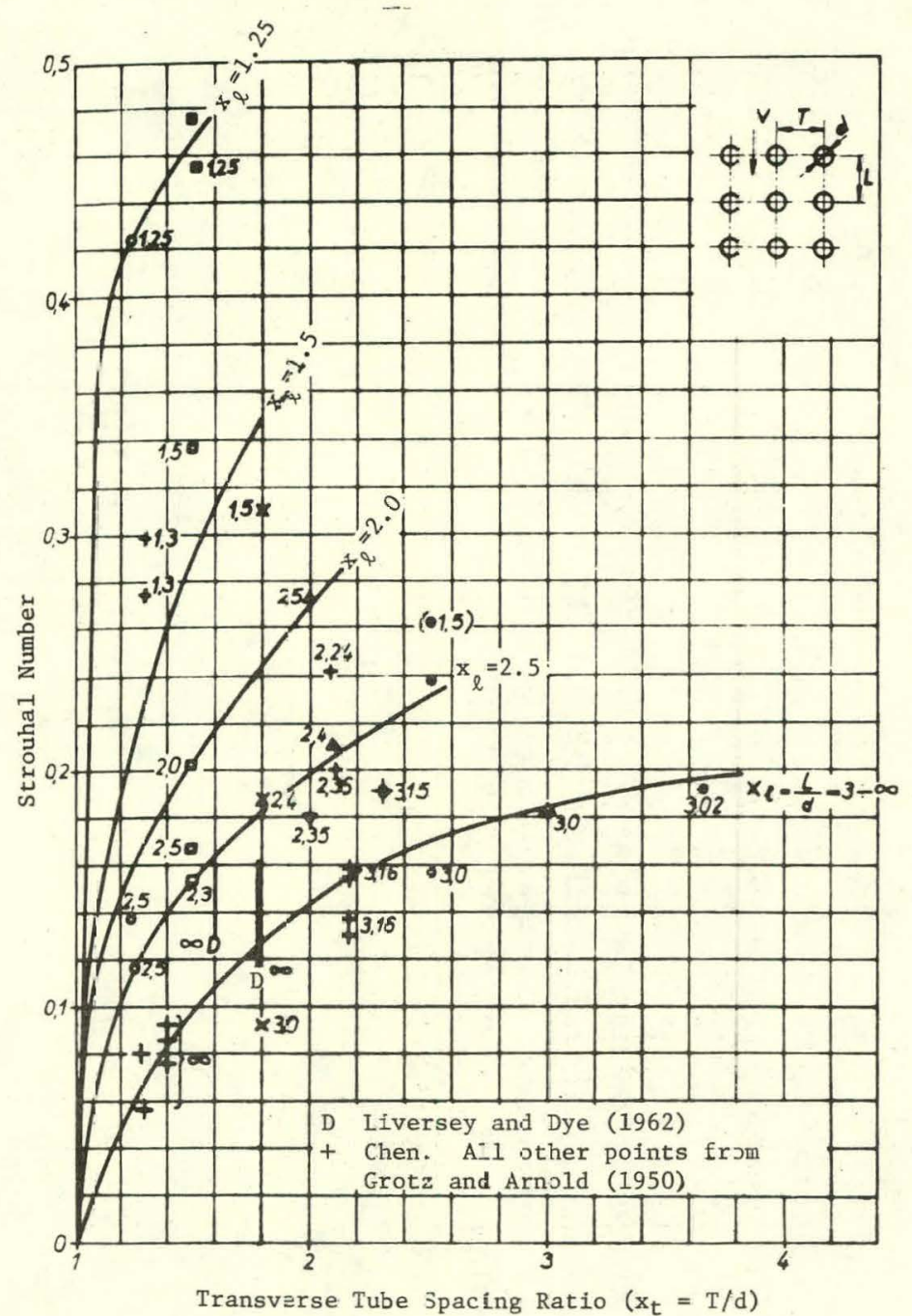

Fig. 17. Strouhal Number vs Transverse Tube-spacing Ratio with Longitudinal Tuke-spacing Ratio $\left(x_{\ell}=\mathrm{L} / \mathrm{d}\right)$ for In-line Tube Banks. (From Ref. 24.)

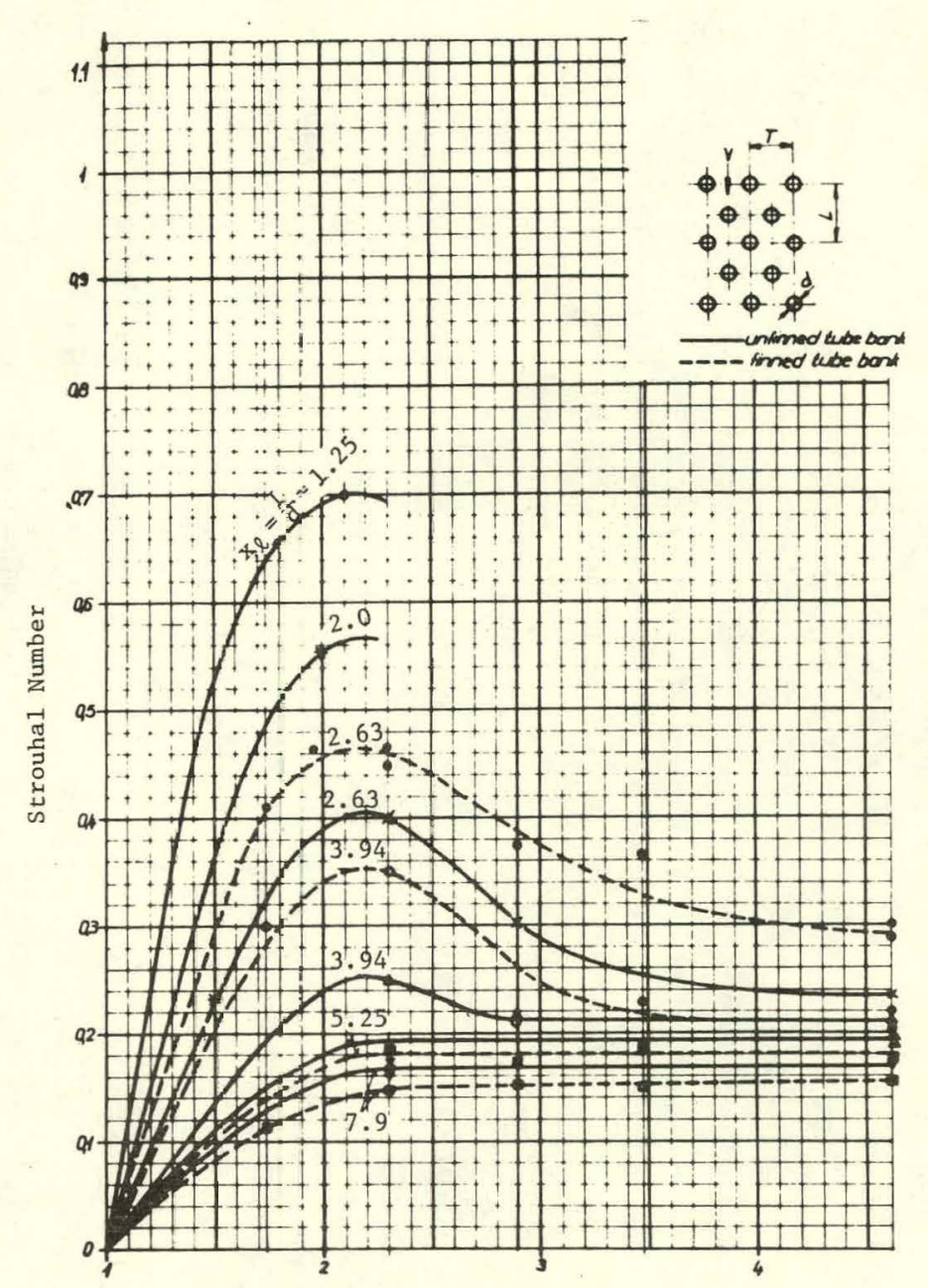

Transverse Tube Spacing Ratio $\left(x_{t}=T / d\right)$

Fig. 18. Strouhal Number vs Transverse Tube-spacing Ratio with Lo.zgitudinal Tube-spacing Ratio $\left(x_{\ell}=L / d\right)$ for Staggered Tube Banks. (From Ref. 24.) 


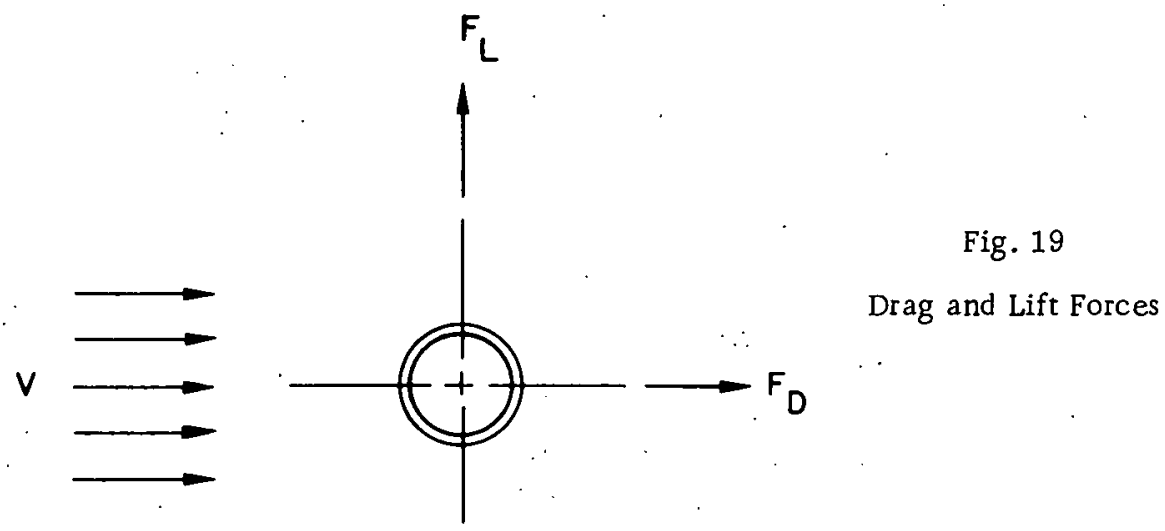

oscillates periodically or randomly about zero. The frequency of this oscillating drag force is twice that associated with the shedding of a vortex pair (the frequency in the lift direction).

In the lift direction, one cycle is the interval between two vortices shed consecutively from the same side of the tube; during that interval, one vortex sheds off from the other side of the tube. In the drag direction, the cycle is the interval between each vortex shed, irrespective of the tube side.

A dimensional analysis shows that the magnitude of the drag and lift forces exerted on a cylinder can be expressed in the following forms: ${ }^{4}$

Drag force: $F_{D}=C_{D} \frac{1}{2} \rho \mathrm{AV}^{2} ;$

Lift force: $F_{L}=C_{L}^{\frac{1}{2}} \rho A^{2}$.

where

$F_{D}, F_{L}=$ time-averaged drag force and lift force, respectively,

$C_{D}, C_{L}=d r a g$ and lift coefficients, respectively,

$\rho=$ mass density of fluid,

$A=$ projected area per linear length of cylinder $(=d)$,

and

$$
\mathrm{V}=\text { free-stream velocity. }
$$

Many diversified experiments reporting values of $C_{D}$ and $C_{L}$ are available in the literature. However, specific values of $C_{D}$ and $C_{L}$ cannot be assigned with certainty because of the wide variation in measured values. Lienhard $\mathrm{d}^{4}$ reviewed the experimentally determined data on drag and lift forces, and plotted the values of the drag and lift coefficients over a wide range of Reynolds numbers for an isolated single stationary cylinder. Some of his results are summarized below. 
a. Drag Coefficient $\left(C_{D}\right)$. The drag force $F_{D}$ has two force components: pressure drag $\left(F_{D_{p}}\right)$ and frictional drag $\left(F_{D_{f}}\right)$. The pressure drag results from pressure distribution over the surface of the body; the frictional drag results from the viscous shear force on the surface. For a given flow velocity, the friction drag is relatively constant, while the pressure drag contains a constant component and an oscillatory component. The oscillatory component is associated with vortex shedding. Experimental results indicate that this oscillatory component is relatively small, compared to the constant components. At very low Reynolds numbers, the drag will consist almost entirely of $\mathrm{F}_{\mathrm{f}}$. As the oscillating force appears, $F D_{f}$ gradually becomes negligible in comparison with $\mathrm{FD}_{\mathrm{p}}$. Lienhard $\mathrm{d}^{4}$ plotted many investigators' test data, giving the values of drag coefficients shown in Figs. 20 and 21 for two Reynolds-number ranges. Various coefficients are used in higher-Reynoldsnumber regimes; they include: $C_{D}$, which is based upon the time-averaging value of FD; $C_{D r m s}$, which is based upon the root-mean-square variations of $F_{D} ; C_{D_{\max }}$, which is based upon the maximum absolute values of $F_{D}$; $C_{K D}$, which is based on the harmonic-drag-force oscillation about the mean; and $C_{D_{p}}, C_{D_{f}}$, and $C_{D_{o}}$, which are based upon $F_{D_{p}}, F_{D_{f}}$, and the amplitude of the drag-force oscillation respectively. These figures show that at the lowReynolds-number regime $(0.5<R e<300)$, the mean drag coefficient $\left(C_{D}\right)$ varies from 20 to 1 , and at the high-Reynolds-number regime $(300<\operatorname{Re}<$ $2 \times 10^{5}$ ), a nearly constant value of $C_{D}=1.1$ is preserved, while the oscillatory drag coefficient $\left(C_{D_{0}}\right)$ is in the range of $0.1-0.2$. In the transition range, the drag coefficient drops drastically.

\section{b. Tift. ronefficient (C.L). A lift force is totally aseociatcd with} the vortex shedding and is therefore oscillatory. Figure 22 shows the results of the measured mean oscillatory lift coefficients compiled by Chen. ${ }^{37}$ Most of the measurements were made in the Reynolds-number range $10^{4}<\operatorname{Re}<10^{6}$. The scatter of the data is large, and the data are far less consistent than the drag-coefficient data; values vary from 0.1 to 1.5 , with the use of $1.5-2.0$ for the value of $C_{L}$ considered conservative. In tube-response analysis, only the vibratory excitation is of interest. Since the excitation in the lift direction is 5 to 10 times higher than the excitation in drag direction (because of the greater force coefficient), the lift direction is of primary importance.

For tube arrays, $C_{L}$ appears to depend on tube spacings as well as Reynolds number. Chen ${ }^{37}$ presented some measured data of $C_{L}$ with respect to the longitudinal tube-spacing ratio $\left(x_{\ell}\right)$ and transverse tube-spacing ratio $\left(x_{t}\right)$. These data indicate that the values of $C_{L}$ are lower than 0.5 for closely packed tube arrays. However, it is premature to draw any conclusions at this time.

\section{Vibrating Tubes}

Problems become further complicated when considering vibrating cylinders and the correlation length. The latter is defined as a length of the cylinder over which the vortex shedding process is coherent or consistent; this 


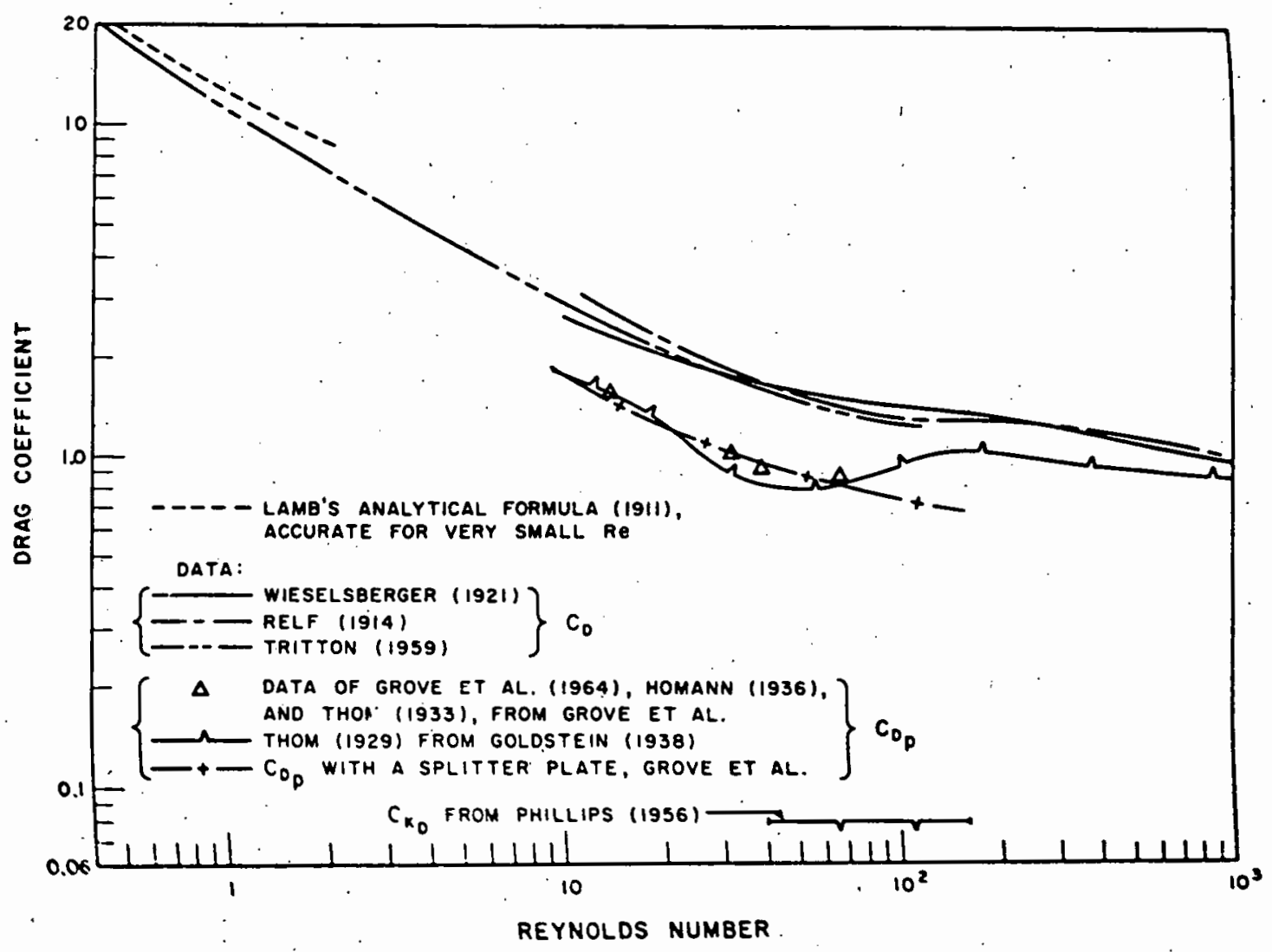

Fig. 20. Drag Coefficients for Rigid Circular Cylinders: $0.4 \leq \mathrm{Re}<10^{3}$. (From Ref. 4.)

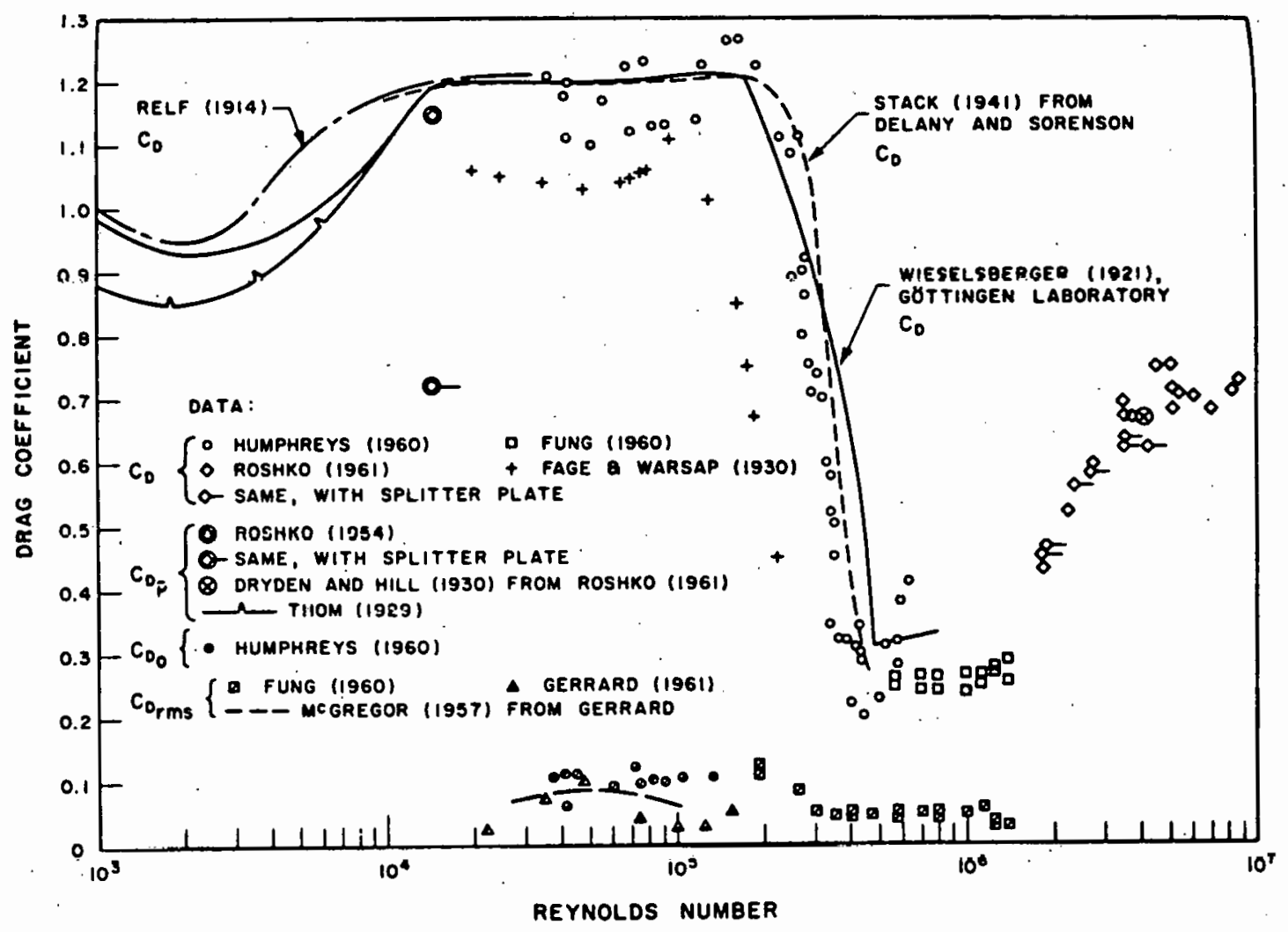

Fig. 21. Drag Coefficients for Rigid Circular Cylinders: $10^{3} \leq \operatorname{Re}<10^{7}$. (From Ref. 4.) 


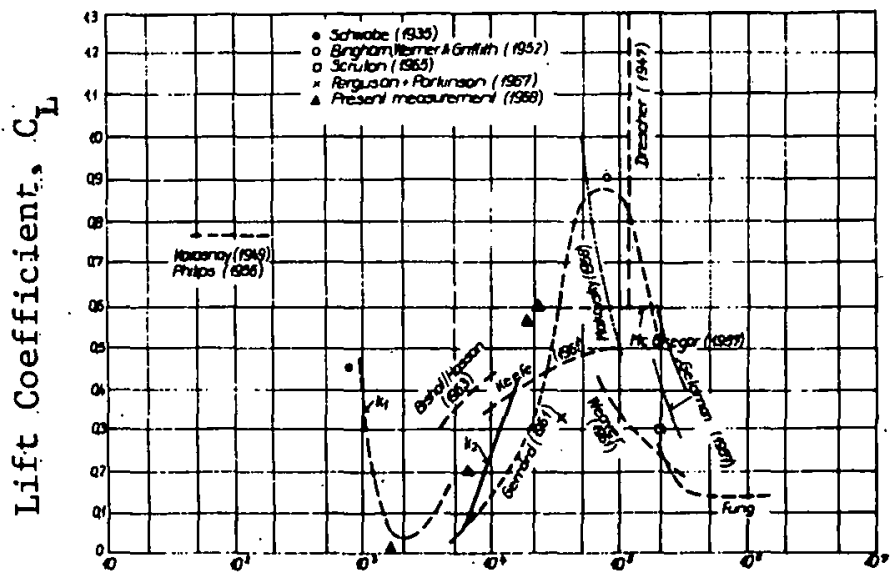

Fig. 22

Experimentally Determined Mean Oscillatory Lift Coefficients for a Single Stationary Cylinder. (From Ref. 37.)

Reynolds Number.

length is typically 6-10 diameters. If the length of the cylinder is longer than the correlation length, the $\mathrm{drag}$ and lift forces acting on the cylinder are not in phase over the entire span. ${ }^{t 0}$ 'l'he vortex-shedding phenomena of a vibrating cylinder has been studied at some length by Marris ${ }^{3}$ and Den Hartog. ${ }^{41}$ They reported that the intensity of the lift force is greater for a cylinder vibrating at the Strouhal shedding frequency than for a cylinder at rest. Nelms and Segaser, ${ }^{1}$ in summarizing the NADC (Naval Air Development Center) test results ${ }^{42}$ on flexible cables towed through water, suggest a steady drag coefficient of 1.35 for use as a conservative estimate of $C_{D}$ when a vibrating-tube condition exists. In general, the interaction between the elastic tubes and vortex shedding is not yet understood clearly.

\section{Acoustic Standing Waves}

The steady crosiflow over tube arrays in the inlet/outlet zones of shell-side fluid in the steam generator can also induce an acoustic standing wave perpendicular to both tubes and flow. This standing wave, in resonance with natural frequencies of tubes or shell; can cause extensive structural damage and a high shell-side pressure drop.

For a cylindrical shell, Morse $e^{43}$ gives the transverse acousticstanding-wave resonant frequency $\left(f_{a}\right)$ as:

$$
f_{\mathrm{a}}=\pi \alpha_{\mathrm{m}} \frac{\mathrm{c}}{\mathrm{d}_{\mathrm{s}}} \text {, }
$$

where

$$
\begin{aligned}
c & =\text { velocity of sound in fluid, } \\
d_{S} & =\text { inner diameter of cylindrical shell, }
\end{aligned}
$$

and

$\alpha_{\mathrm{m}}$ depends on the mode excited: 


$\begin{array}{ccccc}\text { Mode } & \frac{\alpha_{\mathrm{m}}}{1} & & \frac{\text { Mode }}{5} & \frac{\alpha_{\mathrm{m}}}{2.042} \\ 2 & 0.5861 & 6 & 2.388 \\ 3 & 0.9722 & 1.337 & 7 & 2.730 \\ 4 & & 1.693 & 8 & 3.071\end{array}$

Fitz-Hugh ${ }^{44}$ suggested that if the shell-side liquid is gaseous, an acoustic resonance with vortex shedding should be checked. The uncertainty in vortex-shedding frequency $f_{\mathrm{V}}$ makes acoustic resonance possible, even if $0.5 \mathrm{f}_{\mathrm{a}}<\mathrm{f}_{\mathrm{v}}<2 \mathrm{f}_{\mathrm{a}}$. Acoustic resonance can be suppressed by placing longitudinal baffles along the flow direction or by. omitting some tubes in a tube array to disturb the regular flow pattern. ${ }^{45,46}$ A resonance between the vortex-shedding frequency and the acoustic standing wave, while it may result in a loud noise, will not generally cause tube damage unless the tube natural frequency is also coincident.

In general, acoustic resonance will not be a problem in LMFBR steam generators for several reasons:

a. In a liquid, acoustic pressure amplitudes are too small to have significant effects on tube vibration. ${ }^{6}$

b. The velocity of sound in a liquid, being significantly greater than that in a gas, will generate a standing-wave frequency that is generally much greater than the vortex-shedding frequency.

c. Shell diameters, being relatively small, also result in high standing-wave frequencies. In this regard, Cohen and Deane ${ }^{47}$ report that; even in gaseous systems, acoustic resonance is generally only a problem in large units having a transverse width greater than $22 \mathrm{ft}$.

B. Turbulent Buffeting

When a flow crosses an isolated single cylinder, vortex shedding occurs within certain ranges of Reynolds numbers. However, in closely packed. tube arrays, vortex shedding may no longer occur. Owen ${ }^{48}$ stated that, deep within a bank of tubes, the cumulative growth of random irregularities in labyrinth-like, high-Reynolds-number flow must lead to a state of almost complete incoherence on which it is difficult to imagine any superposed regular pattern (apart from a general drift through the bank) to be discernible. Owen explained the tube vibrations sufficiently deep inside the bank by the randomly fluctuating turbulent forces as follows:

Starting with a simple hypothesis that the dominant frequency of the turbulent fluctuations is identifiable with the dominant frequency of the fluctuating force on the tubes, the former is proportional to the average flow velocity 
through the bank. As this velocity is increased from zero, two resonant conditions may arise: one when the dominant frequency coincides with a natural frequency of the tube, the other, an acoustic resonance when shell-side fluid is gaseous. Theoretical considerations led to the equation

$$
\frac{f_{b} L_{1}}{V_{1}} \frac{T}{d}=K\left(1-\frac{d}{T}\right)^{2},
$$

where

$f_{b}=$ dominant turbulent buffeting frequency,

$L_{1}=$ distance between centerlines of successive tube rows (see Fig. 23),

$T=$ transverse spacing of tubc (sec Fig. 23),

$\mathrm{d}=$ outer diameter of tube,

$\mathrm{V}_{1}=$ mean flow velocity between adjacent tubes,

and

$$
\mathrm{K}=\text { constant. }
$$

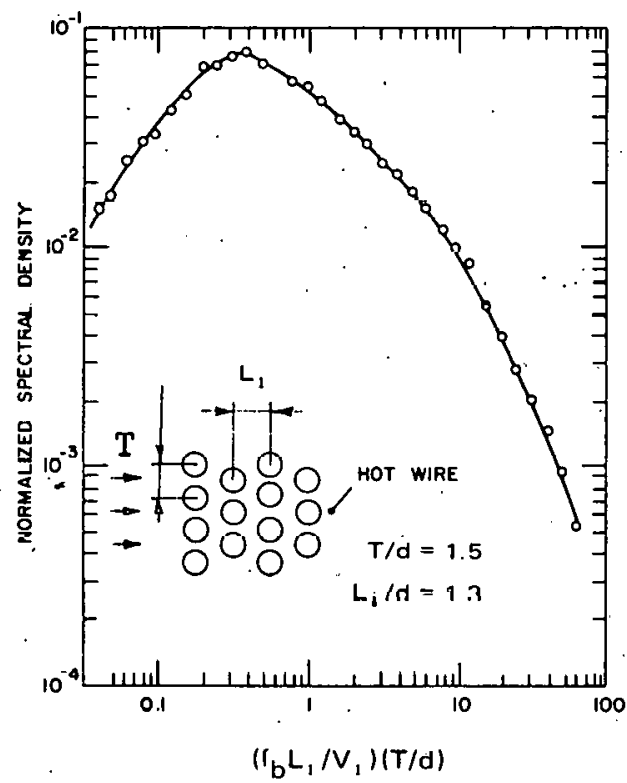

Fig. 23

Typical One-dimensional Turbulent-energy Spectrum Determined Experimentally. (From Ref. 48.)

Owen suggested the value of $K=3.05$ based on limited experimental data. He also proposed a rule for estimating $f_{b}$ : The dominant frequency of the vibration in a bank of tubes, for which the ratio of the diameter to the lateral spacing lies between 0.2 and 0.6 , is equal to the interstitial flow velocity divided by twice the distance between successive rows. That is,

$$
f_{b}=\frac{V_{1}}{2 L_{1}} .
$$


Multiplying both sides of Eq. 7 by d/ $V_{1}$ yields the Strouhal-number relationship:

$$
\frac{f_{b d}}{V_{1}}=\frac{d}{2 L_{1}}
$$

Owen also included an experimentally determined one-dimensional turbulent-energy spectrum, reproduced in Fig. 23, which shows that the pronounced peak occurs near $\left(\mathrm{f}_{\mathrm{b}} \mathrm{L}_{\mathrm{l}} / \mathrm{V}_{1}\right)(\mathrm{T} / \mathrm{d})=0.4$ for the tube spacings $\mathrm{T} / \mathrm{d}=$ 1.5 and $L_{l} / d=1.3$. However, as reported by Wambsganss, ${ }^{16}$ this result does not agree with the empirical relationship given by Eq. 7 which implies that the peak in the turbulent-energy spectrum should occur at $\left(f_{b_{b}} L_{1} / V_{1}\right)(T / d)=0.75$.

Wambsganss also noted that the actual peak location can be translated into a Strouhal number of 0.21 , which corresponds to the Strouhal number associated with vortex shedding from a single tube. This correspondence may be a coincidence, or it may be that the peak in the turbulent-energy spectrum for crossflow through tube banks occurs at a constant Strouhal number of 0.2 (single-tube value for vortex shedding). If the latter is true, a mechanism for tube vibration based on buffeting can be postulated that agrees with the vibration problems that have occurred and that would have been "predicted" by the proximity of the tube Strouhal numbers to a flow Strouhal number of about 0.2. As described by Wambsganss, this mechanism is as follows:

a. A dominant frequency is associated with the turbulent-energy spectrum at the flow velocity, which can be characterized by a Strouhal number $\left(\mathrm{f}_{\mathrm{b}} \mathrm{d} / \mathrm{V}_{\mathrm{l}}\right)$ of 0.2 .

b. If the flow velocity through the tube bank is such that the dominant frequency of turbulent fluctuations corresponds to a natural frequency of tube vibrations (i.e., if the tube Strouhal number is close to 0.2 ), a type of resonance occurs. This resonance is sharpened by the selectivity of the tube in responding to that group of eddies whose dimensions are comparable with the tube diameter. ${ }^{48}$

c. The resulting increased amplitude of vibration caused by the "resonant" condition generates vortices at the frequency of oscillation (by virtue of the tube's motion through the fluid) and the phasing necessary to continue the vibration, giving rise to a type of vibration self-excitation.

d. The process is reenforcing, with the potential of leading to largeamplitude destructive vibrations and eventual tube failure.

As discussed in Ref. 16, the concept is intuitively appealing, with Owen's work providing insight and initial experimental evidence to support the hypothesis. Owen's development is based on gas flow and has not been verified in liquids. 
C. Fluidelastic Whirling

When a flow crosses single- and multirow tube arrays, and the flow exceeds a certain critical value, a large whirling vibration of the tubes is possible, as shown by Connors. ${ }^{49}$ The vibrations are of a self-excited or fluidelastic nature in that, once initiated, the vibration amplitude will grow even to cause tube impact and severe tube damage. Connors explained this fluidelastic excitation mechanism as the momentary displacement of one tube in an array from its normal equilibrium position, altering the flow field, thereby upsetting the force balance on neighboring tubes and causing them to also change their positions in a vibratory manner. If, during a cycle of vibration, the energy extracted from the flowing fluid by the tubes exceeds the energy dissipated by damping, a fluidelastic vibration is established. Connors characterized the threshold velocity above which fluidelastic instabilities occur.

\section{Connors' Threshold Velocity}

Based on wind-tunnel test results from a single row of smooth cylinders $(\mathrm{T} / \mathrm{d}=1.41)$, coupled with quasi-static measurements of fluid forces, Connors showed the stability boundary to be defined by the empirical relationship

$$
\frac{V_{1}}{f_{n} d}=9.9\left(\frac{m_{0} \delta_{0}}{p d^{2}}\right)^{1 / 2}
$$

where.

$$
\begin{aligned}
\mathrm{V}_{1}= & \text { mean flow velocity between adjacent tubes, } \\
\mathrm{f}_{\mathrm{n}}= & \text { natural frequency of tube, } \\
\mathrm{d}= & \text { outer diameter of tube, } \\
\mathrm{m}_{0}= & \text { virtual mass of tube per unit length (equals the mass of the tube } \\
& \text { itself, plus the added mass of the displaced fluid), } \\
\delta_{0}= & \text { logarithmic decrement of tube in still fluid; dimensionless, }
\end{aligned}
$$

and

$$
\rho=\text { mass density of fluid. }
$$

His experimentally determined stability diagram is shown in Fig. 24.

By rearranging Eq. 9, we can express the threshold velocity $\left(\mathrm{V}_{\mathrm{T}}\right)$ in a general form by 


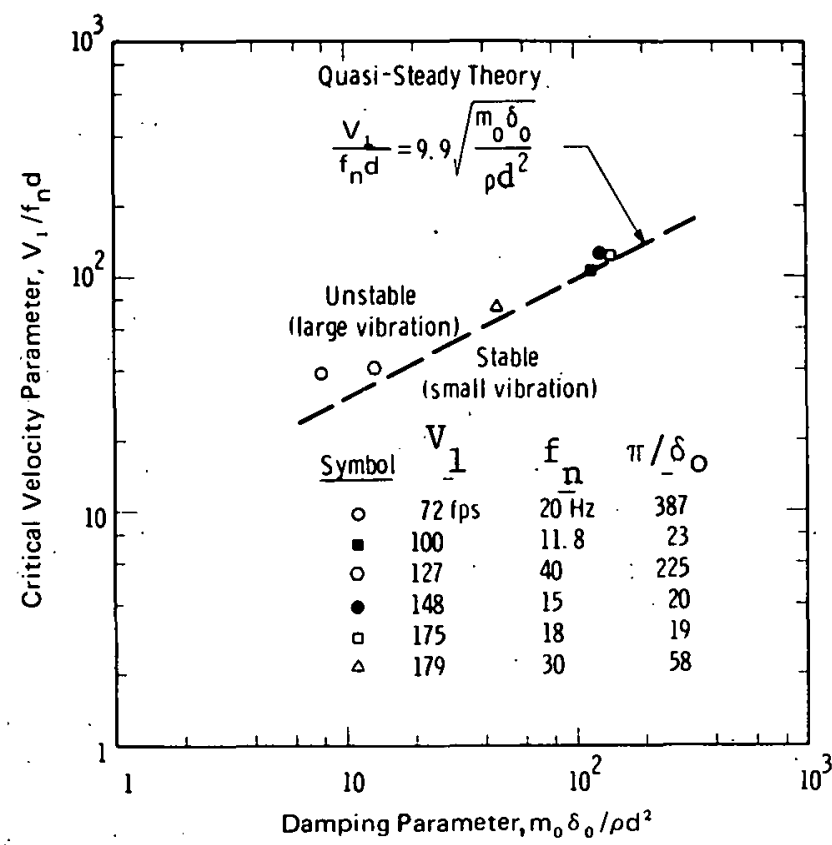

Fig. 24. Stability Diagram for Single-row Arrays Having a Pitch-to-Diameter Ratio of 1.41. (From Ref. 49.)

$$
\mathrm{V}_{\mathrm{T}}=\mathrm{K}_{\mathrm{T}^{\mathrm{f}}}\left(\frac{\mathrm{m}_{0} \delta_{0}}{\rho}\right)^{1 / 2},
$$

where $\mathrm{K}_{\mathrm{T}}$ is proportionality factor, which, for Connors' experiment, is 9.9 .

After Connors' investigation of fluid instabilities in wind-tunnel tests, several other author $s^{50-53}$ also investigated this instability experimentally, using different tube patterns and spacings and fluids other than air: liquids and two-phase (air and water) mixtures. Pettigrew ${ }^{51}$ plotted his test results with others, as shown in Fig. 25. Two data points from singlerow water-flow tests by Halle and Laurence ${ }^{52}$ have been added (assuming a log-decrement damping value of $0.1)$, and are in good agreement with their results for a liquid. Also shown

are experimental results ${ }^{53}$ from airflow tests on overhung spans of finned $\mathrm{U}$-tubes simulating a sodium-to-air heat exchanger. For this geometry and spacing, the proportionality factor is about 15. It appears that the proportionality factor is near 6.6 for liquid and two-phase mixtures. Hartlen ${ }^{22}$ pointed out that the factor $\left(f_{n} d\right)^{-1}$ is approximately proportional to the tubespan-to-diameter ratio, which does not vary greatly for typical designs; thus, $\mathrm{V}_{1} / f_{\mathrm{n}} \mathrm{d}$.depends primarily on the shell-side flow velocity. The fluidelastic instability boundary in liquid flow appears to overlap with that for vortex shedding, as shown in Fig. 25. Therefore, it may not be possible to detect two distinct mechanisms in practice.

Because the fluidelastic instability boundary and reduced velocity associated with vortex shedding overlap for a "liquid system," steam generators designed to have tube natural frequencies greater than the calculated vortex-shedding frequency are generally also safe from the standpoint of fluidelastic excitation. However, since the proportionality factor depends on tube-bundle geometry and spacing, the results given in Figs. 24 and 25 should be used for design guidance only.

Blevins ${ }^{54}$ recently developed a two-dimensional analytical model for fluidelastic whirling of a tube row in crossflow, based on the assumptions that the tubes are not excited by vortex-shedding and jet-switching mechanisms. His conclusions on model studies are as follows:

a. The critical velocity is a function of the natural frequencies and damping factors of tubes in both coordinate directions. 


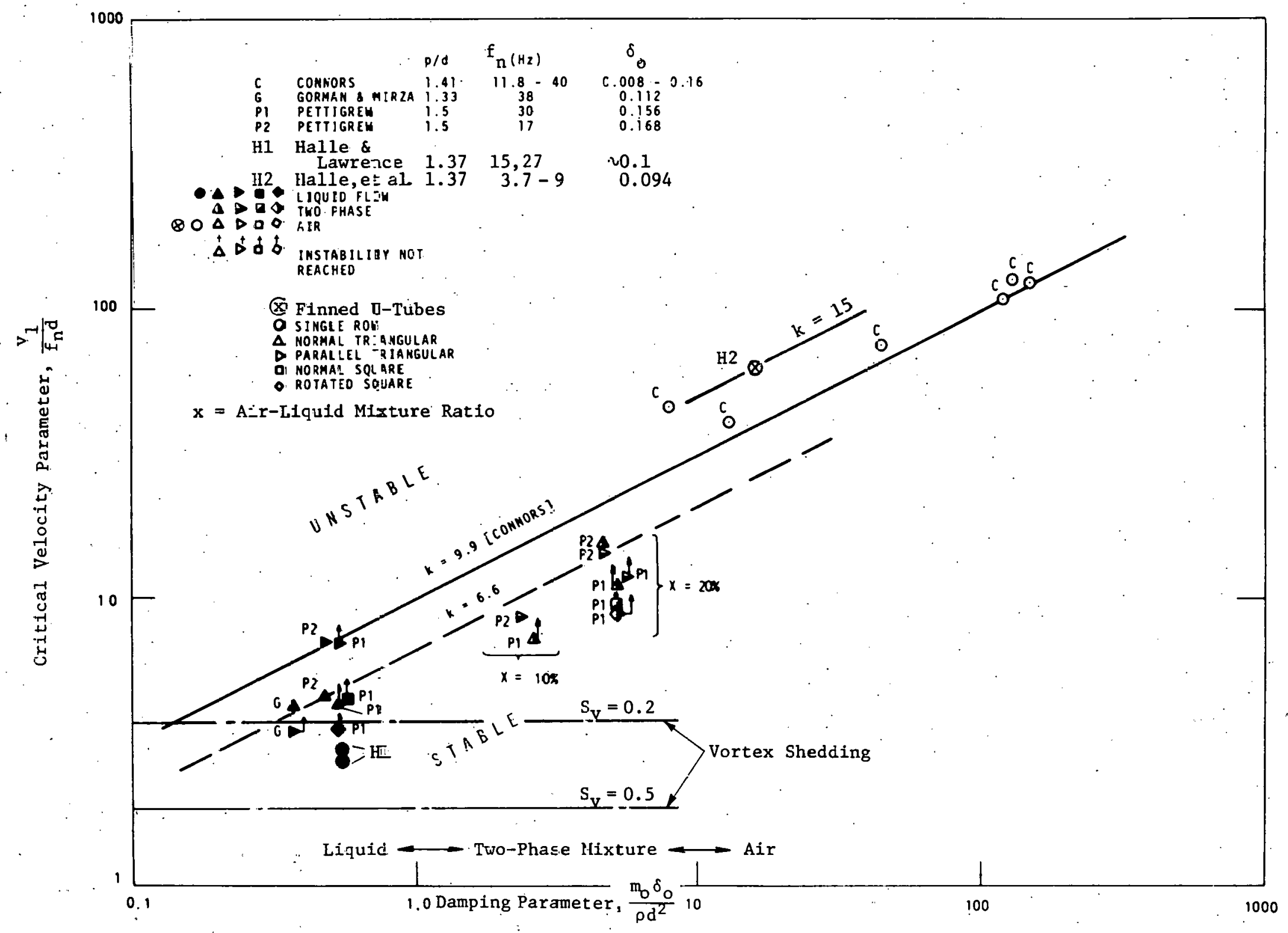

Fig. 25. Stability Diagram for Tube Arrays. (From Ref. 51, with $\mathrm{B} 1$ and H2 added.) 
b. The critical velocity increases sharply with the separation of the natural frequencies of adjacent tubes.

c. The critical velocity increases as the number of tubes in the row decreases.

d. A tube row bounded on one side by a wall and on the other side by a fixed tube can have a lower critical velocity than if the same tube row were bounded by fixed tubes.

\section{Jet Switching}

When a flow crosses a closely packed tube array, the fluid forms jets as it isisues from the gap between the tubes. Roberts ${ }^{55}$ first observed a jetswitching mechanism in a single-row tube array. Later, Connor $\mathrm{s}^{49}$ also investigated the mechanism in connection with fluidelastic whirling of tubes. Connors noted that the jet-switching mechanism is of secondary importance in the self-excited vibration of tube arrays. Roberts and Connors explained this mechanism as follows.

If tubes are widely spaced, giving a small solidity ratio,* the individual jet remains parallel. If the solidity ratio is about 0.45 or greater, a jet pair. appears as shown in Fig. 26a. When the tubes are displaced a sufficient amount, alternately one upstream and one downstream, the jet pairing can be switched back and forth as shown in Fig. 26b.

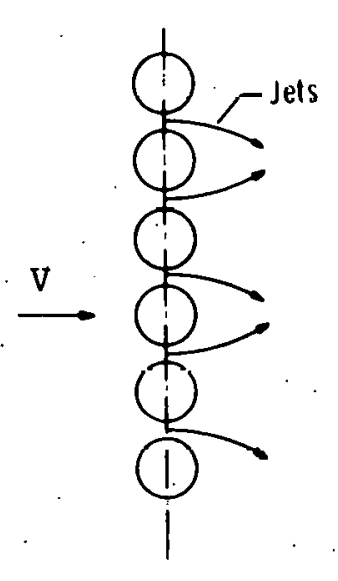

(a) Jet Pairing in an Aligned Row

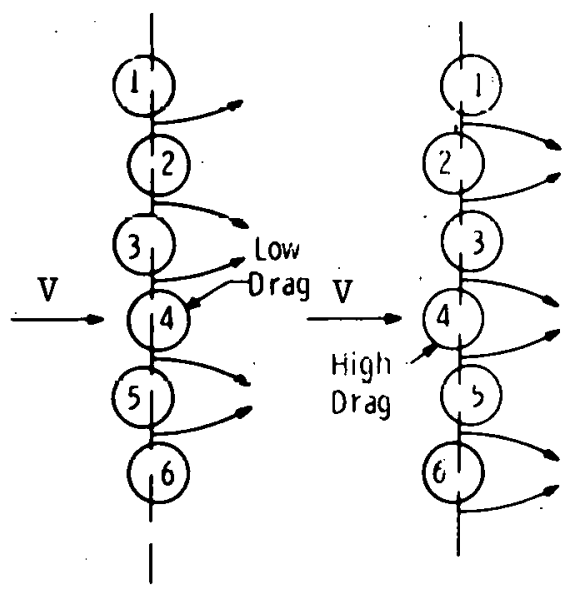

(b) Switching of Jet Pairing Caused by Streamwise Displacement of Tubes

Fig. 26. Jet Pairing from a single Row of Tubes. (From Ref. 49.)

Roberts observed, and Connors confirmed, that jet switching is accompanied by an oscillatory drag force with amplitude variations of about $20 \%$. Connors noted that a wide wake (diverging jets) behind a tube corresponds to

*The ratio of the cylinder-filled portion of the cross section to the total cross section. 
a lower drag force, while a narrow wake (converging jets) corresponds to a higher drag force. Roberts reported there is a finite time involving entrainment of fluid by the jets that prevents jet switching if $V_{1} / f_{n} d<75$.

\section{E. Turbulent Pressure Fluctuations in Parallel Flow}

Parallel-flow-induced vibration problems had not been considered in reactor or steam-generator component design until Burgreen et al. ${ }^{56}$ experimentally investigated rod vibration in axial flow. Later, Paidoussis 57-59 formulated an equation of motion and performed a stability analysis. He correlated analytical results with experimental test results to illustrate the phenomenon of fluidelastic instability of tubes that can occur when axial flow velocities exceed certain critical values. Chen ${ }^{60-69}$ extended Paidoussis' work to include, among other things, a study of the effects of support conditions, Coriolis force, pulsating flow, and in-plane and out-of-plane stability of curved tubes conveying fluid. The critical velocities are sufficiently high to exclude such instabilities in steam generators. ${ }^{57,59}$ At subcritical flow velocities, the predominant effect of the flowing fluid is to reduce the natural frequency of vibration and to give rise to a fluid-excitation mechanism associated with the random pressure fluctuations in the turbulent boundary layer.

Prediction of response to turbulent wall-pressure fluctuations is more difficult than the stability problem, since it requires mathematical characterization of the forcing function. Several authors ${ }^{70-75}$ have developed methods of predicting tube-displacement responses with a probabilistic approach using. random vibration techniques. In these studies, the excitation mechanism has been identified as the random pressure fluctuations generated by turbulent eddies in the flow. Many experimental test results are available, ${ }^{76-83}$. giving the statistical properties of the wall-pressure fluctuations beneath a turbulent boundary layer. Results from these studies are used in a phenomenological model for the random pressure field used in developing an equation to predict response. ${ }^{72}$ Application is limited by lack of information on the pressure field: specifically, the effects of upstream disturbances and adjacent structures.

\section{F. Hydraulic Noise}

Hydraulic noise, which refers to the absolute pressure fluctuations traveling through the loop system, may cause vibrations and associated tube fretting. Card ${ }^{84}$ investigated the effect of hydraulic noise on nuclear-fuel vibration by building a quiet loop in which various types and levels of hydraulic noise are added upstream of the tube bundles, using noise generators. He observed that tube vibration is sensitive to hydraulic-noise characteristics, and that less pressure-tube fretting occurred in a horizontal arrangement than in a vertical one. 


\section{VIBRATION ANALYSIS}

A difficult task the nuclear industry has faced for some time is the prediction of vibration characteristics and response of steam-generator internal components to reduce the possibility for vibration-induced failures. Presently, it is difficult to accurately predict the dynamic behavior of steamgenerator components because of the limitations of current technology and the lack of experimental results. This section describes available analytical procedures, based upon state-of-the-art technology, for evaluating the vibration characteristics and response of steam-generator components. The important considerations in performing a vibration analysis are outlined here. Details of selected items are discussed in Secs. A-E below.

(1) Shell-side flow velocities. Shell-side cross- and parallel-flow velocities must be estimated at typical areas. The critical regions are shellside inlets/outlets, bend regions, the first span away from shell-side inlets/ outlets (transition region), and a typical parallel-flow region. Critical areas may arise in different positions other than specified above because flow distributions depend upon the mechanical arrangement of the system; such critical areas remain to be identified.

(2) Added mass. When a structure vibrates in a fluid, the fluid gives rise to a two-part fluid-reaction force, which can be interpreted as an added mass and damping contribution to the dynamic response of the structure. When a single tube is immersed in an infinite fluid medium, the virtual mass of the tube is the mass of the tube itself plus the added mass of fluid displaced by the tube. ${ }^{75}$ For a closely spaced tube array, the concept of added mass. must be extended to an added mass matrix that is a function of tube spacing and/or adjacent boundaries. ${ }^{85,86}$ Added mass can significantly reduce component natural frequencies and must be included in a vibration-response analysis.

(3) Reynolds number. Reynolds numbers at the regions identified in Sec. V.A below should be calculated before any vibration-response analysis is performed, because vibration analysis parameters are expressed as a function of Reynolds number (see Sec. IV).

(4) Natural frequency of tube vibration. A fundamental need in any vibration analysis is to estimate the component natural frequencies, since resonant conditions with exciting frequencies and fluidelastic instabilities must be avoided. Both in-plane and out-of-plane vibration in bend regions must be included, along with the effects of baffle-hole clearances and baffle thickness on tube vibration. Axial forces and creep can also affect the natural frequencies.

(5) Vortex shedding. Vortex shedding and lift and drag forces were discussed in Sec. IV.A. Vortex-shedding frequencies must be separated from 
the natural frequencies of the tubes to avoid a resonant condition. Nelms and Segase ${ }^{1}$ suggested a separation factor of 3 if flow velocities cannot be predicted accurately. To compute response, drag and lift forces must be estimated. The corresponding static deflection of a tube must be calculated and combined with others, if any. Assuming the tube may vibrate out of phase, the maximum resultant deflection must not exceed half of the gap between the tubes to avoid tube-to-tube collision.

(6) Turbulent buffeting frequency. Turbulent buffeting was discussed in Sec. IV.B. To avoid resonant conditions, the dominant buffeting frequency must be separated from natural frequencies of the tubes.

(7) Fluidelastic instabilities in crossflow. Fluidelastic instabilities in crossflow were discussed in Sec. IV.C. When a crossflow velocity exceeds a certain critical value, a large whirling vibration of tubes is possible. The threshold velocity, dependent on damping and tube-bundle geometry, should be calculated and shown to be significantly less than design flow velocities to avoid large-amplitude vibrations of the tubes.

(8) Jet-switching mechanism. The jet-switching mechanism was discussed in Sec. IV.D. For a closely packed tube array, the fluid forms a jet as it issues from the gap between the tubes. The induced gap velocity must be less than threshold level to avoid jet-switching effects.

(9) Damage-number concept. Thorngren ${ }^{87}$ considered two types of vibration-damage sources: tube-to-baffle impact and tube-to-tube collision. His "damage numbers" are based on the assumptions that the lift-force coefficient equals 3 , there is $40 \%$ contact area between tube and baffle, and the induced shear stress is less than the fatigue stress.

(10) Fluidelastic instabilities in parallel flow. As discussed in Sec. IV.E, large-amplitude vibrations of tubes are possible when a parallel-flow velocity (shell side or tube side) exceeds a certain critical velocity. In present steamgenerator designs, the critical velocity is sufficiently high that such instabilities will not occur.

(11) Prediction of subcritical vibration amplitude in parallel flow. Small-amplitude tube vibrations are possible even at flow velocities much smaller than the critical velocities for fluidelastic instabilities. Several existing methods can be used to estimate such subcritical vibration amplitudes.

(12) Acceptance criteria. It is difficult to specify a complete set of acceptance criteria applicable to any steam-generator design. However, as a minimum, it is generally required that (1) the maximum combined stresses associated with each excitation mechanism and other forms of loading must not exceed the endurance limit, to avoid fatigue failure; (2) the maximum expected displacement must be less than half the minimum gap between tubes, 
to avoid tube-to-tube impacting; and (3) in predominantly crossflow regions, the natural frequency of tube must be sufficiently greater than both the maximum expected vortex-shedding frequency and the critical frequency associated with fluidelastic whirling to ensure that resonance and fluidelastic instability, respectively, will not occur. Despite satisfying the above criteria, fretting and wear at the tube-support locations can be a problem.

\section{A. Shell-side Flow Velocities}

The shell-side fluid-flow velocities must be determined before flowinduced vibration analysis because analysis parameters are functions of these velocities. The flow velocities vary across the tube bundle and also along the tube span. The state of the art is inadequate to predict accurate shell-side distributions.

Tinker ${ }^{88}$ suggested that the average shell-side crossflow velocities be computed as the volumetric shell-side flow rates divided by average crossflow areas such that

$$
\mathrm{v}_{0}=\frac{\mathrm{W} / \mathrm{p}_{0}}{\mathrm{~A}_{0}}
$$

where

$$
\begin{aligned}
& \mathrm{V}_{0}=\text { mean shell-side flow velocity, } \\
& \mathrm{W}=\text { shell-side mass flow rate, } \\
& \rho_{0}=\text { weight density of fluid, }
\end{aligned}
$$

and

$$
\mathrm{A}_{0}=\text { average } \operatorname{cros} \text { fllow area. }
$$

The average crossflow areas $\left(A_{0}\right)$ at the centerline of a circular cylindrical unit are given as lollows: ${ }^{88}$

For a triangular tube pattern, $A_{0}=\alpha \frac{g}{p} D_{B} l$

$$
\left(\begin{array}{ll}
\rightarrow^{0} & 0 \\
0 & 0
\end{array}\right)
$$

for a. square tube pattern, $A_{0}=\alpha \frac{g}{p} D_{B} l$

$$
\left(\rightarrow \begin{array}{ll}
0 & 0 \\
0 & 0
\end{array}\right)
$$


for a rotated square tube pattern, $A_{0}=1.414 \alpha \frac{g}{p} D_{B} l$

(Contd.)

$$
\left(\begin{array}{lll}
\rightarrow & 0 & \\
& 0 & 0 \\
& & 0
\end{array}\right)
$$

where

$$
\begin{aligned}
\alpha & =\text { constant }\left(\text { Tinker }^{88} \text { suggests a value of } 0.97\right) \\
g & =\text { gap between adjacent tubes } \\
\mathrm{p} & =\text { pitch of tubes } \\
\mathrm{D}_{\mathrm{B}} & =\text { outer-diameter limit of tube bundle, }
\end{aligned}
$$

and

$$
l=\text { length of tube span between tube supports. }
$$

In E.qs. 11 and 12, the area between the shell and the tube bundle is neglected and only $97 \%$ of gap area between the tubes is used. The average shell-side parallel-flow velocities could be calculated in a similar manner. There is no established method for computing the flow velocities; velocities range from 2 to $20 \mathrm{ft} / \mathrm{sec}$ in present steam generators.

\section{B. Added Mass}

When a tube array is immersed in a shell-side fluid medium, the added mass of the tube is no longer the mass of fluid displaced by the tube, but it is affected by adjacent tubes and the shell-side wall or shroud that surrounds the tube bundle. The added mass can be expressed in general by

$$
\mathrm{n}_{\mathrm{f}}=\mathrm{C}_{\mathrm{M} \rho} \frac{\pi \mathrm{d}^{2}}{4}
$$

where

$$
\begin{aligned}
m_{f} & =\text { added mass of fluid external to tube per unit length, } \\
\mathrm{C}_{M} & =\text { added mass coefficient, } \\
\rho & =\text { mass density of fluid, }
\end{aligned}
$$

and

$\mathrm{d}=$ external diameter of tube.

If a single tube is immersed in an infinite fluid medium, $C_{M}=1$. In other words, the added mass of the tube is the mass of fluid displaced by the tube. 
The added mass of the tube in a compressible-fluid annulus (see Fig. 27) has been reported by Chen and Wambsganss, ${ }^{75}$ and their expression of $\mathrm{C}_{\mathrm{M}}$ is

$$
C_{M}=\frac{\left[b J_{0}(b)-J_{1}(b)\right] Y_{1}(a)-\left[b Y_{0}(b)-Y_{1}(b)\right] J_{1}(a)}{\left[b Y_{0}(b)-Y_{1}(b)\right]\left[a J_{0}(a)-J_{1}(a)\right]-\left[b J_{0}(b)-J_{1}(b)\right]\left[a Y_{0}(a)-Y_{1}(a)\right]^{\prime}}
$$

where

$$
\begin{aligned}
\mathrm{J}_{\mathrm{i}}, \mathrm{Y}_{\mathrm{i}} & =\text { Bessel functions of the first and second kinds, of order } \mathrm{i}, \\
\mathrm{a} & =\omega \mathrm{d} / 2 \mathrm{c} \\
\mathrm{b} & =\omega \mathrm{D} / 2 \mathrm{c} \\
\omega & =\text { vibration frequency, } \\
\mathrm{c} & =\text { velocity of sound in fluid, } \\
\mathrm{d} & =\text { outer diameter of tube, }
\end{aligned}
$$

and

$D=$ diameter of rigid duct to confine the fluid annulus.

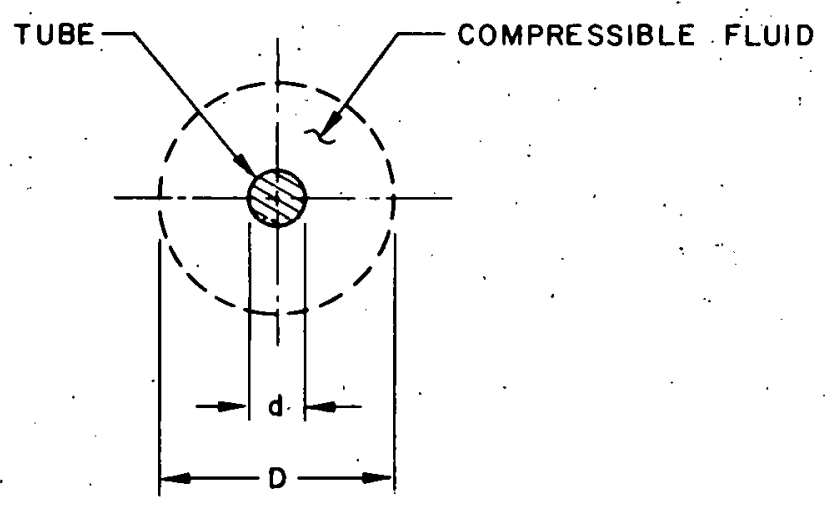

Fig. 27

Tube in a Compressible-fluid Annulus

Chen and Wambsganss explained the added mass of a tube in a compressible fluid annulus as follows:

The added mass coefficient $C_{M}$ depends on the frequency, sound velocity, diameter of tube and duct, as can be seen from Eq. 14. Results of computation for various diameter ratios (Fig. 28 and $\mathrm{Table}$ II) show that the added mass coefficient $C_{M}$ is insensitive to the parameter $a=\omega d / 2 c$. In general, a is small; therefore, the fluid may be assumed to be incompressible. Figure 14 shows that the boundary effect is important when the tube is relatively close to the duct wall, i. e. $; \mathrm{D} / \mathrm{d}<3$.

If a tube is immersed in an incompressible fluid annulus, Eq. 14 reduces to

$$
C_{M}=\frac{(D / A)^{2}+1}{(D / d)^{2}-1}
$$




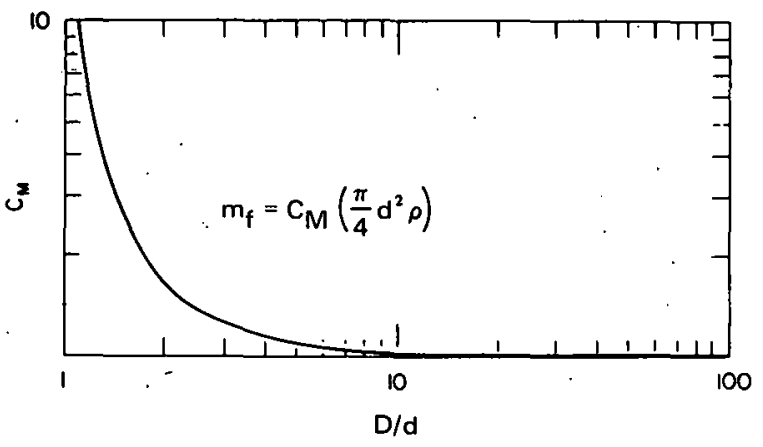

Fig. 28. Added Mass Coefficients $\left(C_{M}\right)$ for $\mathrm{V}$ arious Rod/Wall-diameter Ratios and $\mathrm{a}=0.001$. (From Ref. 75.)
TABLE II. Added Mass Coefficient $\left(C_{M}\right)$ for Various Rod/Wall-diameter Ratios (D/d)

\begin{tabular}{rrrr}
\hline & \multicolumn{1}{l}{${ }^{a}$} & & \\
$\mathrm{D} / \mathrm{d}$ & 0.00001 & 0.001 & \multicolumn{1}{l}{0.1} \\
\hline 1.02 & 50.50873 & 50.50894 & 51.03145 \\
1.04 & 25.51164 & 25.51169 & 25.77994 \\
1.06 & 17.18247 & 17.18247 & 17.36652 \\
1.08 & 13.02014 & 13.02013 & 13.16222 \\
1.10 & 10.52454 & 10.52456 & 10.64147 \\
1.2 & 5.54548 & 5.54549 & 5.54615 \\
1.4 & 3.08334 & 3.08335 & 3.12611 \\
1.6 & 2.28206 & 2.28206 & 2.28241 \\
1.8 & 1.89287 & 1.89287 & 1.92497 \\
2.0 & 1.66667 & 1.66667 & 1.69715 \\
4.0 & 1.13333 & 1.13334 & 1.16431 \\
6.0 & 1.05714 & 1.05715 & 1.09265 \\
8.0 & 1.03175 & 1.03175 & 1.07267 \\
10.0 & 1.02020 & 1.02021 & 1.06807 \\
\hline
\end{tabular}

The effecl of fluid viscusily un the added masis and danping of a rud vibrating in a fluid annulus was also evaluated in a theoretical/experimental study. ${ }^{89}$

The added mass of a cylinder confined by hexagonal arrays of six similar cylinders was studied experimentally in Ref. 89. A method of analysis has been developed at ANL for calculating the added mass tensor of a group of circular cylinders vibrating in a liquid. ${ }^{85,86}$ The method can be applied to

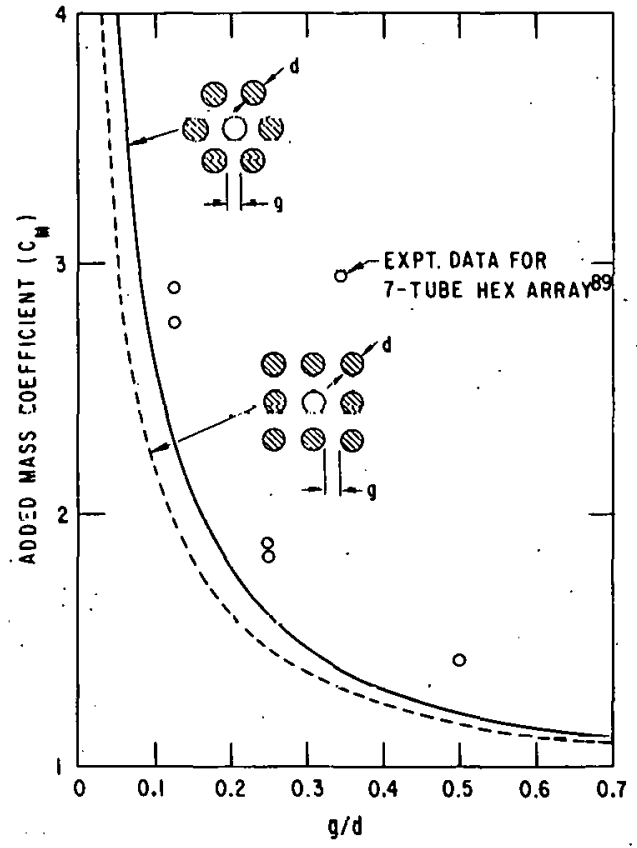

Fig. 29. Added Mass Coefficients for Seven-tube Hexagonal Array and Nine-tube Square Array. (From Refs. 85, 86, and 89.) a group of circular cylinders arranged in any tube pattern, and the cylinders may have different diameters. Typical results for a seventube hexagonal array and a nine-tube square array are shown in Fig. 29.

C. Natural Frequency of Tube Vibration

To avoid detrimental tube vibration, resonant conditions must be suppressed by ensuring separation of natural frequencies of the tubes and exciting frequencies. Many techniques are available for computing natural frequencies of tubes. For example, the tube can be assumed to be a continuous beam intermediately supported by baffles, with tubcsheets at both ends. State-of-the-art techniques can be applied on the assumption that intermediate baffles act like "knife-édged hinge" supports. However, in reality, baffles have a certain thickness, and inherent tube-to-baffle hole clearances are associated with manufacturing tolerances. Several author $\mathrm{s}^{21,23,90-92}$ studied 
the effects of these parameters on tube vibrations; however, it is premature to draw any general conclusions. The axial force and long-term creep phenomena can also affect the natural frequencies. Creep apparently has a negligible effect on altering the vibration characteristics of the tubes in current steam generators. 16

\section{Single-span Straight Beam}

The natural frequencies of bending vibration of a uniform tube can be calculated from ${ }^{93}$

$$
f_{n}=\frac{\lambda_{n}}{2 \pi} \sqrt{\frac{E I}{m_{0} l^{4}}}
$$

where

$$
\begin{aligned}
f_{n} & =\text { natural frequency of } n \text {th mode of tube, } \\
E & =\text { modulus of elasticity, } \\
I & =\text { moment of inertia, } \\
l & =\text { length of beam between supports, } \\
m_{0} & =\text { virtual mass of beam per unit length; }
\end{aligned}
$$

and

$\lambda_{\mathrm{n}}=$ frequency factor of nth mode.

The virtual mass of the tube $m_{0}$ equals the sum of three mass cumponents:

$$
m_{0}=m_{t}+m_{f}+m_{i}
$$

where

$$
\begin{aligned}
& \mathrm{m}_{\mathrm{t}}=\text { mass of tube per unit length, } \\
& \mathrm{m}_{\mathrm{i}}=\text { mass of fluid inside tube per unit length, }
\end{aligned}
$$

and

$\mathrm{m}_{\mathrm{f}}=$ added mass of fluid external to tube per unit length (see Sec. IV.B).

The frequency factor $\lambda_{\mathrm{n}}$ depends on the end conditions, and is a function of the dimensionless axial force $(\Gamma)$ given by

$$
\Gamma=\frac{F_{A} \ell^{2}}{E I},
$$


where $F_{A}$ is the axial force applied to the tube. Chen and Wambsganss ${ }^{94}$ tabulated values of $\lambda_{n}$ for the first five natural frequencies in the range of $\Gamma$ from -10 to 10 , with three types of end conditions: both ends fixed, both ends simply supported, and one end fixed with the other end simply supported. Some of these values are listed in Table III to show the extent to which the natural frequency of a tube increases with axial tension and decreases with axial compression.

TABLE III. Frequency Factor $\left(\lambda_{n}\right)$ of a Single-span

Straight Beam with Three Types of End Conditions (From Ref. 94)

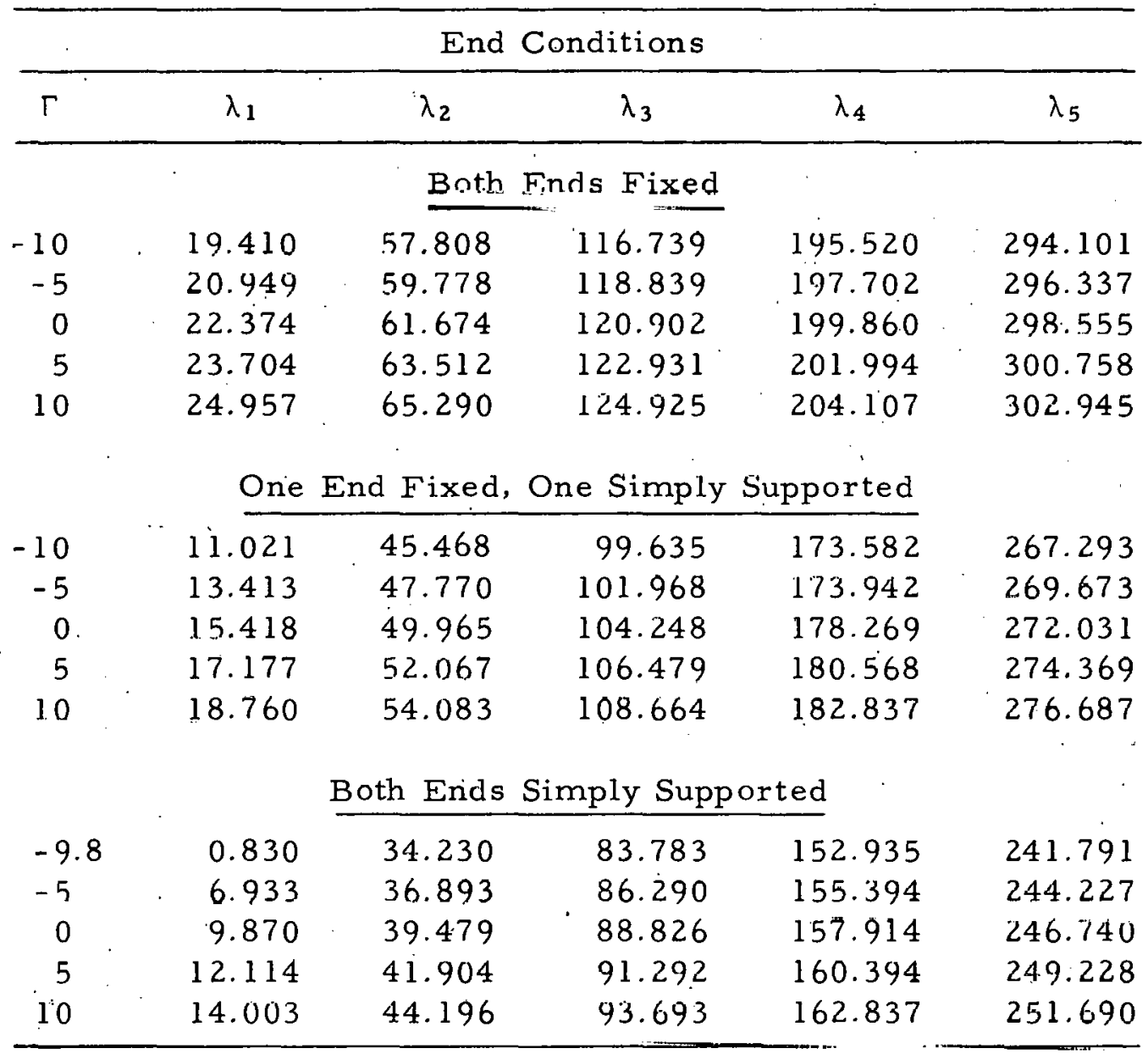

2. Single-span Curved Beam

Whenever the central line of the unstressed beam is a plane curve and its plane is a principal plane of the beam at each point, the vibrations of a curved beam can be classified as flexural vibrations of the beam in its plane, and flexural vibrations involving both displacement at a right angle to the plane of beam and twist. For steam-generator tubes, the in-plane and out-of-plane motions are uncoupled. 
The frequencies of a uniformly curved beam are obtained from

Ref. 95:

$$
f_{n}=\frac{\lambda_{n}}{2 \pi}\left(\frac{1}{R \theta}\right)^{2} \sqrt{\frac{E I}{m_{0}}}
$$

where $R$ is the radius of curvature, and $\theta$ is the subtended angle (in radians).

a. In-plane Motion. The beam is considered to be inextensible; inextensional theory predicts that the fundamental mode is asymmetric. In reality, this is true for a large subtended angle. When $\theta$ is small, the fundamental frequency is associated with the first symmetric mode, and the frequency factor may be approximated by that for a corresponding straight beam whose length is the same as the arc length of the curved beam.

The frequency factors for in-plane motion based on the inextensional theory are given in Ref. 94 and plotted in Fig. 30.

b. Out-of-plane Motion. The frequency factor for out-of-plane motion depends on the subtended angle $\theta$, the stiffness ratio $r$, and support conditions. The stiffness ratio is given by

$$
r=\frac{G J}{E I}
$$

where $\mathrm{J}$ is the polar area moment of inertia of the beam, $\mathrm{C}$ is the shear modulus of elasticity, and the other parameters are the same as those of straight beams. For a circular or square cross section,

$$
\mathbf{r}=\frac{1}{1+\eta}
$$

therefore $\mathbf{r}$ depends on Hoisson's ratio $\Pi$ only. Frequency factors for $\|=0.3$ and three types of end conditions are given in Ref. 94 and plotted in Fig. 31.

\section{Multiple-span Beams}

Several different methods of analysis can be used to calculate the natural frequencies of continuous beams: the Kayleigh-Ritz method, the matrix-iteration method, ${ }^{96}$ the receptance method, ${ }^{97}$ the wave-propagation method, ${ }^{44}$ the finite-element method, and the conventional method of solving the equation of motion directly. Chen and Wambsgans s ${ }^{94}$ showed a simple graphical method for calculating natural frequencies of straight and curved beams with multiple spans of equal length, using the dynamic three-moment equation. ${ }^{68,69}$ The finite-element method can be used to compute the natural frequencies of unequal span lengths and nonuniform continuous beams, using available computer codes such as NASTRAN and SAP4. ${ }^{98}$ When the finiteelement technique is used, at least three beam elements per span are required to obtain good estimates of the natural frequencies up to the sixth mode. ${ }^{91}$ 


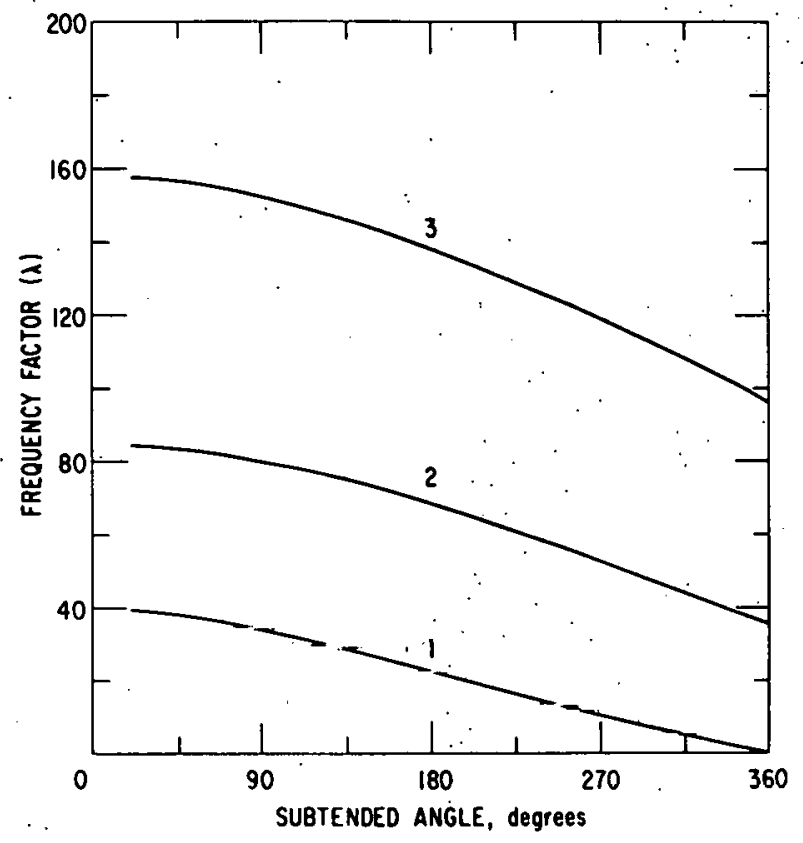

(a) Both Ends Simply Supported

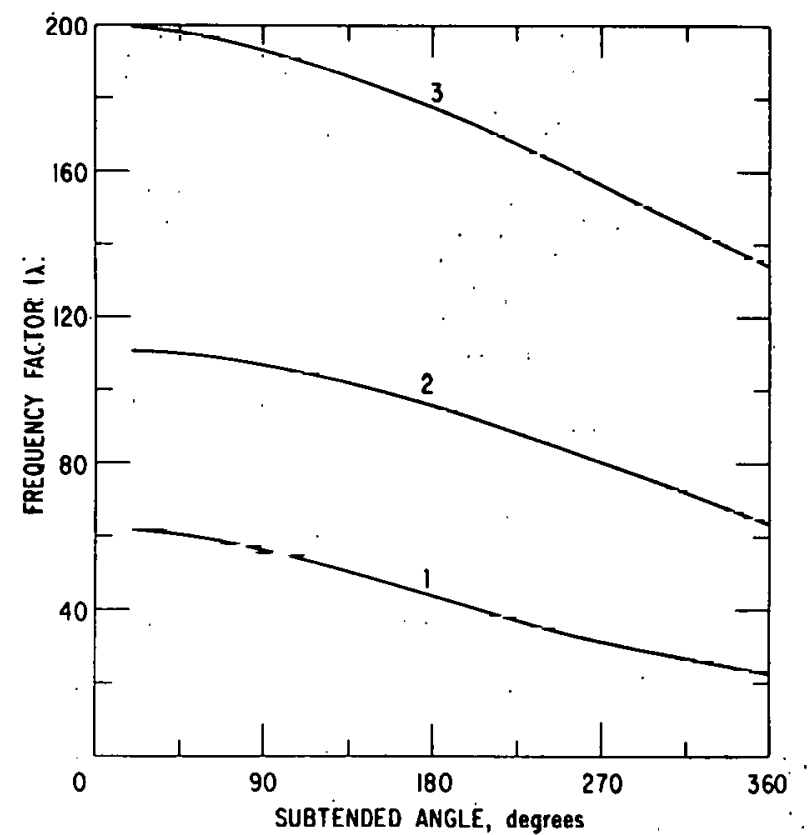

(c) Both Ends Fixed

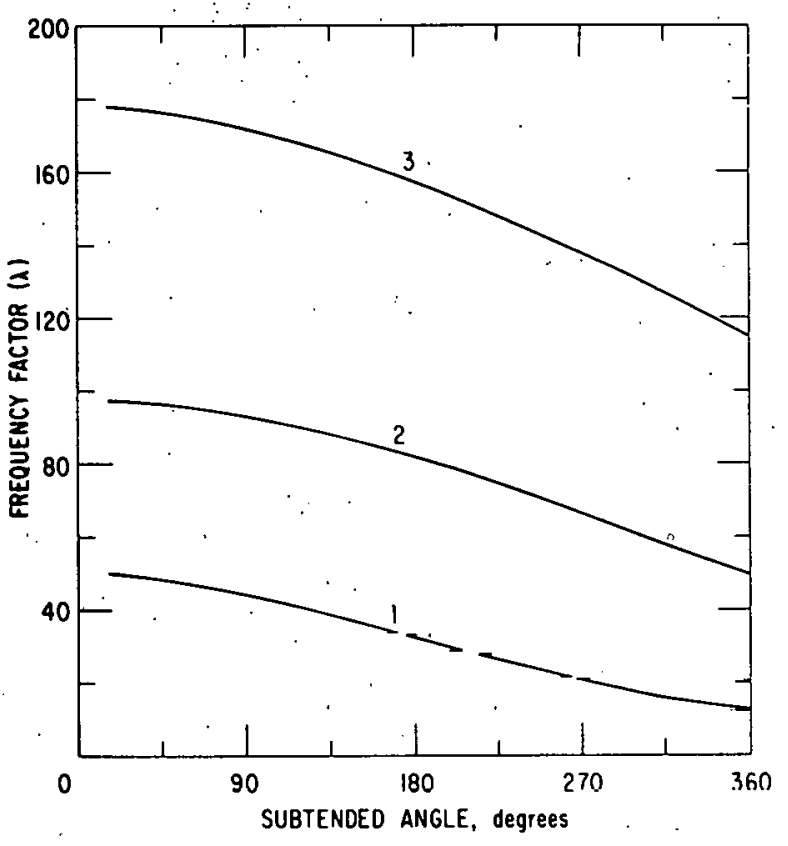

(b) One End Fixed, Other End Simply Supported
Frequency $\mathrm{F}$ actors for In-plane Vibration of Curved Beam with Three Conditions. (From Ref. 94.) 


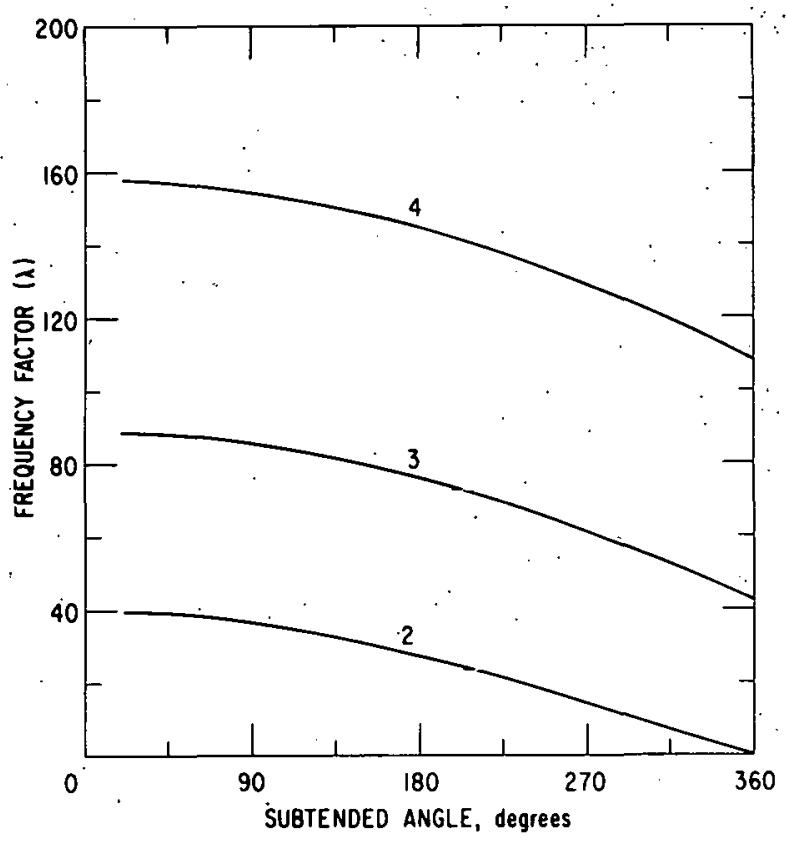

(a) Both Ends Simply Supported

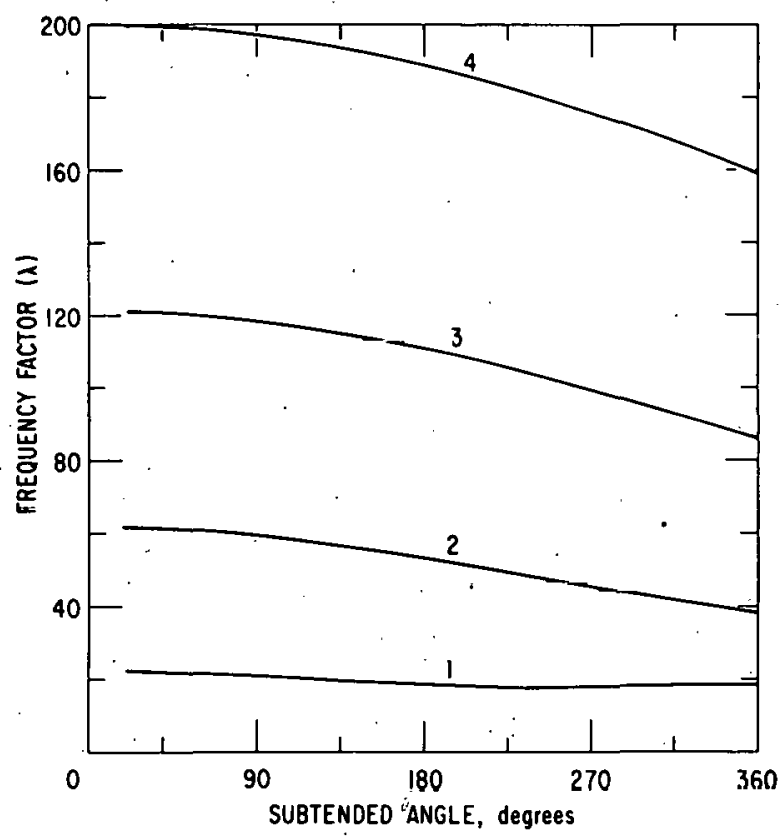

(s) Borh Finds Fixed

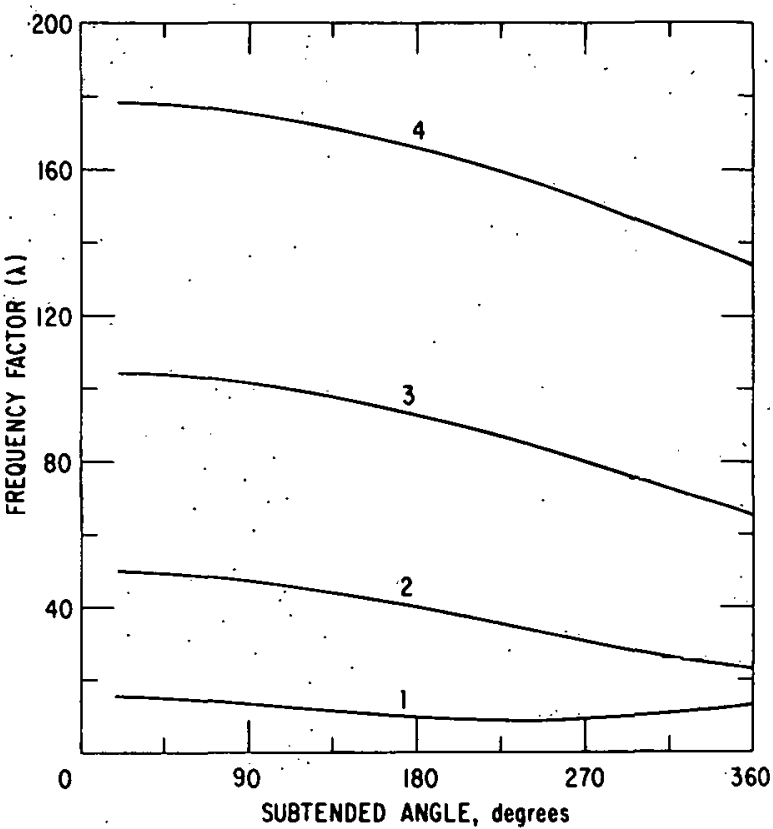

(b) One End Fixed, Other End Simply Supported :

Frequency Factors for Out-of-plane

Vibration of Curved Beam with

Three Conditions. (Frnm Ref. 94.) 
Lee 99,100 also has studied the natural frequencies of heat-exchanger tubes; he concludes that:

a. For a U-bend configuration, torsional flexibility decreases the natural frequencies, and the out-of-plane modes generally have lower frequencies than the in-plane modes.

b. An incomplete ring of a single span would have natural frequencies lower than those of a straight beam of equal length.

c. To maximize the natural frequency, the longest span should be next to the tubesheet. However, if the fluid-dynamic cxcitation acts mainly on that span, there appears to be an advantage to shortening it, resulting in an overall reduction of vibration amplitude.

A survey of heat exchangers showed that the $\mathrm{U}$-bend region of tube bundles experienced the greatest number of tube failures. The flow field in the bend region is not clearly understood. It is therefore suggested lhal, lu avoid vibration problems, the unit should be designed so that there is no appreciable flow in that region. ${ }^{1}$ Vibration suppressors in the form of bars are sometimes mounted in bend regions to reduce the tube vibrations.

\section{Effects of Tube-to-Baffle-hole Clearance and Baffle Thickness}

Sebald and Nobles ${ }^{21}$ conducted a test to study the effect of tube-tobaffle-hole clearance on the natural frequency of tubes using an eight-span. symmetric tube model (Fig. $32 \mathrm{a}$ ); baffle thickness was held constant ( $7 / 8$ in.). Their test results, plotted in Fig. $32 \mathrm{~b}$, show that the natural frequency of a tube decreases as tube-to-baffle-hole clearances increase. The bandwidth of significant amplitudes about the peak appears to increase aboul seven times over that corresponding to zero hole clearance; the increase appeared to be relatively independent of the magnitude of clearance in the range tested.

Moretti and Lowery ${ }^{90}$ studied the effect of baffle thickness on the natural frequency of tubes with constant hole clearance when the baffle is placed near the center of a fixed-fixed beam. Their test results show that the natural frequency of a tube decreases as the baffle thickness increases.

A numerical and experimental study of a cantilevered beam with a motion-limiting stop at the free end ${ }^{23}$ shows that:

a. A single-mode approximation for displacement-response calculation may not be valid for beam/stop interaction.

b. The entire beam rebounds with a velocity proportional to that of the striking velocity.

c. The displacement response of beam/stop impact model is a quasi-narrow band process, whose spectral density curve has a predominant peak. 


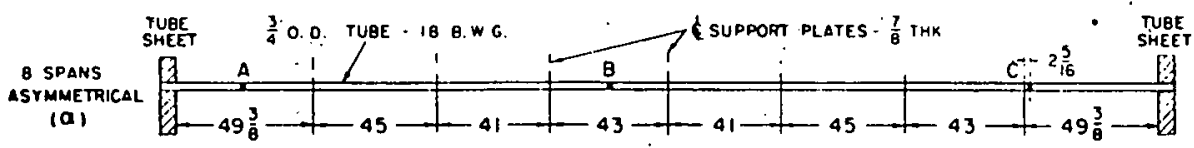

(a) Test Model

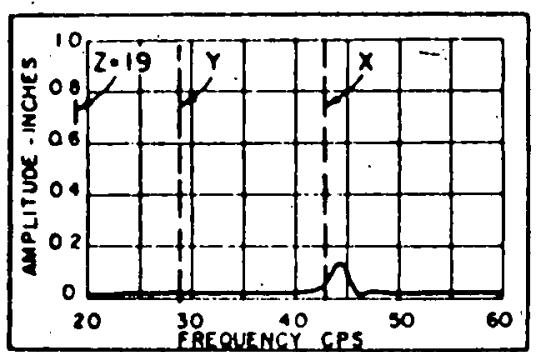

CPS $\triangle T$ PEAK AMPLITUDE - 44.5

RANGE OF FREOUENCY AT

SIGNIFICANT AMPLITUDES $\$ 2.5 \mathrm{CPS}$

ABOUT PEAK

MAXIMUM AMPLITUDE $-0.13^{\circ}$

TUAE HOLE CLEARANCE - O"

RANGE OF NONRESONANT AMPLITUDE. 0.01 "TO $0.02 "$

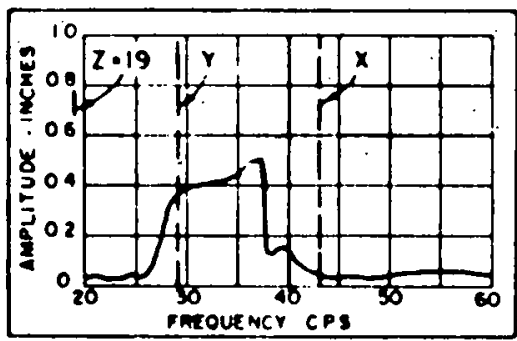

CPS AT PEAK AMPLITUDE - 38

RANGE OF F.REOUENCY AT $]$.

SIGNIFICANT AMPLITUOES $\{17$ CPS

ABOUT PEAK

maXIMUM AMPLITUDE - $0.50^{\circ}$

TUBE hOLE CLEARANCE - 0.010"

RANGE OF NONRESONANT MMPLTUDE. 0.03 " TO 0.06 ".

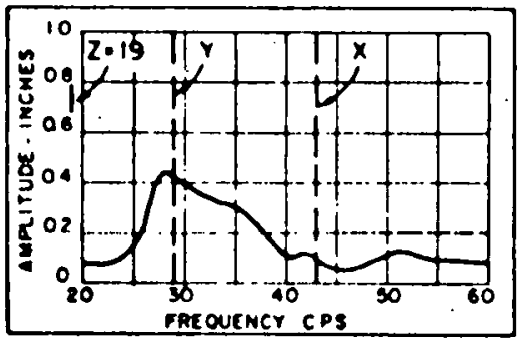

CPS AT PEAK AMPLITUDE - 28

RANGE OF FREOUENCY AT] SIGNIFICANT AMPLITUDES $\} 17$
ABOUT PEAK MAXIMUM AMPLITUDE - $0.44^{\circ}$ TUBE hOLE Clearance - $0.030^{\circ}$ RANGE OF NONRESONANT AMPLITUDE. 0.08 "TO 0.13 ".

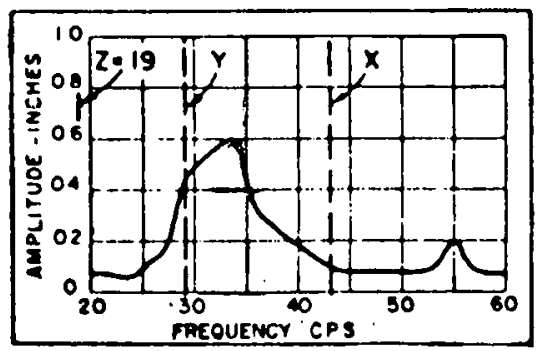

CPS AT PEAK AMPLITUDE - 33.5 RANCE OF FREOUENCY AT SIGNIFICANT AMPUTUOES $\} 10$ CPS ABOUT PEAK

MAXIMUM AMPLITUDE - $0.60 "$

TUBE HOLE CleARANCE - 0.020"

RANGE OF NONRESONANT AMPLITUDE O.06" TO 0.08"

$X=$ Both Ends Fixed, $Y$ - Fixed/Simply Supported,

$Z=$ Both Ends Simply Supported.

(b) Test Results

Fig. 32. Effects of Tube-to-Baffle-hole Clearance on Natural Frequency of Tube. (From Ref. 21.) 
In addition to the tube-to-baffle-hole clearance and baffle thickness, the baffle-hole shape may affect the natural frequency of a tube. Bruce Nuclear Power Station Steam Generator ${ }^{101}$ has trilobar branched holes instead of circular holes; the influence of that geometry on tube vibration characteristics is not known.

\section{Tube-baffle Impact}

Because of the relatively small clearances between tubes and baffle, it is realistic to as sume that if the tubes vibrate at all, some contact or impact will occur. Among other things, the impact affects the vibrational characteristics and response of the tubes; for example, motion between stops effectively increases the frequency of free vibrations. Also, impact forces and velocities are important, because they relate to increased bending stress, contact stress, and weàr.

In regard lo lube-luffle-holc clcarance and damage die to impacting, it is instructive to consider the following illustration: ${ }^{102}$ Assume "damage"

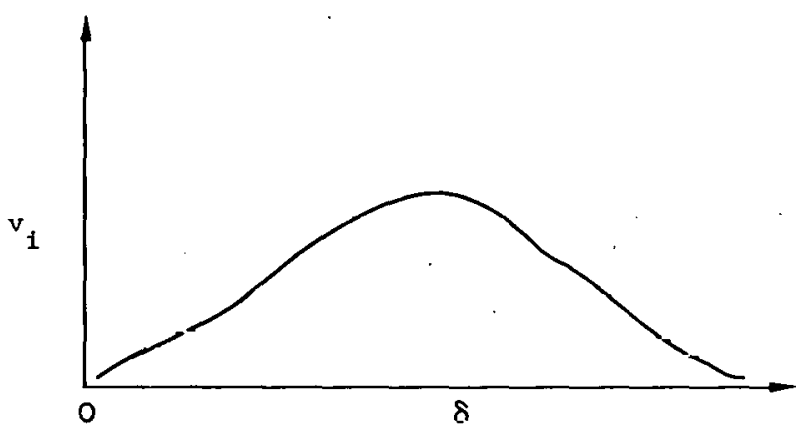

Fig. 33. Hypothetical Curve of Tube Impact Velocity vs Tube-to-Baffle-hole Clearance is proportional to impact velocity $v_{i}$ (tube-to-tube support) which, in turn, is a function of initial clearance $\delta$. A plot of $v_{i}$ versue $\delta$ might take the form of the curve shown schematically in Fig. 33. We can expect $v_{i} \approx 0$ for $\delta=0$, and $v_{i}=0$ for $\delta$ very large (no contact occurs), with a maximum somewhere in between. It is generally assumed that decreasing tube-bafflehole clearance minimizes the potential for impact damage. However, Fig. 33 shows that, with a minimum clearance

set by manufacturing requirements, whether or not designing to a minimum clearance helps depends upon which side of the maximum in Fig. 33 the required minimurn lies.

To gain insight into the impact phenomenon, Chen et al. ${ }^{92}$ theoretically studied a force-excited beam supported at the ends, with motionlimiting stops at the midpoint, and developed a method for solution. Briefly, the method involves determining the "gross". steady-state motion from a single-mode approximation in which the system is reduced to a bilinear vibrating model. Results from the approximation serve as initial conditions in a classical modal analysis in which many modes are included to describe the dynamic stresses and forces resulting from impacting. Finally, a computer code is used to calculate displacement, shearing force, and bending stress of a beam with motion-constraint stops at the midpoint.

D. Damage-number Concept

Two types of vibration damage appear to be prevalcnt in crossflow regions of steam generators: (1) tube-to-baffle impact, and (2) tube-to-tube 
collision. Thorngren ${ }^{87}$ deduced "damage numbers" for the two types of damage, based on the assumptions that the tube is supported by baffles and deflected by a uniformly distributed lift force $\left(C_{L}=3.0\right.$ in Eq. 4$)$.

For tube-to-baffle impact, the baffle damage number has been formulated on the further assumptions that the induced shear stress in the tube at the baffles must not exceed the fatigue stress of the tube material, and that $40 \%$ of the tube cross-sectional area is effective shear area. The baffle damage number $\left(\mathrm{N}_{\mathrm{BD}}\right)$ is given by

$$
N_{B D}=\frac{d \rho V^{2} \ell^{2}}{\beta_{1} S_{m} g_{c} A_{m} B_{t}},
$$

where

$$
\begin{aligned}
\mathrm{N}_{\mathrm{BD}}< & 1 \text { for safe design, } \\
B_{1}= & \text { tube-to-baffle-hole clearance factor, } \\
= & 1.0 \text { for a tube-to-baffle-hole clearance of } 1 / 32 \mathrm{in.}, \\
= & 1.25 \text { for a tube-to-baffle-hole clearance of } 1 / 64 \mathrm{in.}, \\
\mathrm{S}_{\mathrm{m}}= & \text { maximum allowable fatigue stress (ASME Pressure Vessel } \\
& \text { Code, Sec. III), } \\
\mathrm{A}_{\mathrm{m}}= & \text { cross-sectional tube metal area: }\left(\mathrm{d}^{2}-\mathrm{d}_{\mathrm{i}}^{2}\right) \pi / 4 \text {, where d and } \mathrm{d}_{\mathrm{i}} \\
& \text { are outer and-inner diameters of tube, respectively, } \\
\mathrm{g}_{\mathrm{C}}= & \text { gravitational constant, } \\
\mathrm{B}_{\mathrm{t}}= & \text { baffle thickness, } \\
\rho= & \text { midss density of shell-side fluid, } \\
V= & \text { free-stream velocity, }
\end{aligned}
$$

and

$$
l=\text { length of tube between supports. }
$$

For tube-to-tube collision, a collision damage number has been formulated on the further assumption that the maximum deflection of a tube at the midspan should not exceed $50 \%$ of the minimum gap between the adjacent tubes to avoid collision. The collision damage number $\left(\mathrm{N}_{\mathrm{CD}}\right)$ is given by

$$
N_{C D}=\frac{0.625 d \rho V^{2} \ell^{4}}{\beta_{1}^{4} g_{c} A_{m}\left(d^{2}+d_{i}^{2}\right) C_{T} E}
$$

where

$$
\begin{aligned}
\mathrm{N}_{\mathrm{CD}} & <\mathrm{l} \text { for safe design, } \\
\mathrm{C}_{\mathrm{T}} & =\text { minimum gap between adjacent tubes }
\end{aligned}
$$




$$
E=\text { modulus of elasticity, }
$$

and

$\mathrm{d}, \mathrm{d}_{\mathrm{i}}=$ outer and inner diameters of tube, respectively.

Erskine and Waddington ${ }^{103}$ pointed out the weakness of the above equations by examining 19 failure cases of heat exchangers. They suggested the need for a correction factor to the baffle-damage number $\left(\mathrm{N}_{\mathrm{BD}}\right)$. However, the need for a correction factor remains to be verified by examining additional failure data.

\section{E. Prediction of Subcritical Vibration Amplitude in Parallel Flow}

When a tube is placed in a fluid flowing parallel to its axis with flow velocities exceeding a certain critical value, fluidelastic instabilities are possible. ${ }^{57,58}$ However, small-arrplilude lube vilirations are possiblc cven at flow velocities very much smaller than the critical velocities for fluidelastic instabilities. ${ }^{59}$ Such subcritical vibration has the potential for causing damage due to fretting and wear. Several existing methods to predict subcritical vibration amplitudes of tubes are reviewed in this section. Paidoussis ${ }^{104,105}$ has published a critical review on this subject.

\section{Empirical Expression for Peak Amplitude}

Burgreen et al. ${ }^{56}$ were the first to conduct an experiment to investigate vibration of tubes in a fluid flowing parallel to the tube axis. Based on dimensional analysis, they developed an empirical relationship between amplitude of tube vibration, and a number of system parametcrs such as fluid density, flow velocity, geometric and material properties, fluid viscosity, vibration frequency, and hydraulic diameter. The test was conducted with water near room temperature. Quinn ${ }^{106}$ later derived an analytical expression for the amplitude of vibration. Paidoussis ${ }^{107}$ pointed out that the expression contained parameters and constants that were not evaluated, and their relation to the geometry and properties of a given system was not described. Faidous sis ${ }^{107: 1 n R}$ collected the sets of available experimental measurements since Burgreenetal. published their empirical expressiun. Comparison of these data with the expression showed discrepancies up to 10 times for water tests, and up to 100 times for superheated-steam tests. Paidoussis then developed a new empirical expression, based on all available data, which is given by

$$
\frac{\Delta}{d}=\alpha_{1}^{-4} \frac{u^{1 \cdot 6} \epsilon^{1.8} \mathrm{Re}^{0.25}}{1+\mathrm{u}^{2}}\left(\frac{\mathrm{d}_{\mathrm{h}}}{\mathrm{d}}\right)^{0.4} \cdot \frac{\beta^{2 / 3}}{1+4 \beta}\left(5 \times 10^{-4} \times \mathrm{K}_{\mathrm{p}}\right),
$$

where

$$
\begin{aligned}
\mathrm{K}_{\mathrm{p}} & =\text { flow-condition constant } \\
\Delta & =\text { maximum vibration amplitude at midspan }
\end{aligned}
$$




$$
\begin{aligned}
\mathrm{d} & =\text { outer diameter of tube, } \\
\alpha_{1} & =\text { first-mode beam eigenvalue of the tube, } \\
\mathrm{u} & =\text { dimensionless flow velocity }=\left(\mathrm{m}_{\mathrm{f}} / \mathrm{EI}\right)^{1 / 2} \mathrm{Ul}, \\
\mathrm{m}_{\mathrm{f}} & =\text { added mass of fluid external to tube per unit length, } \\
\mathrm{U} & =\text { mean axial flow velocity, } \\
l & =\text { length of tube, } \\
\mathrm{E} & =\text { modulus of elasticity of tube material, } \\
\mathrm{I} & =\text { area moment of inertia of tube section, } \\
\varepsilon & =l / \mathrm{d}, \\
\mathrm{Re} & =\text { Reynolds number, } \\
\mathrm{d}_{\mathrm{h}} & =\mathrm{hydraulic} \text { diameter, } \\
\beta & =\mathrm{m}_{\mathrm{f}} /\left(\mathrm{m}_{\mathrm{f}}+\mathrm{m}_{\mathrm{t}}+\mathrm{m}_{\mathrm{i}}\right), \text { where } \mathrm{m}_{\mathrm{t}}=\text { mass of tube per unit length, }
\end{aligned}
$$

and

$$
\begin{aligned}
\mathrm{K}_{\mathrm{p}} & =\text { flow-condition constant, } \\
& =1 \text { for quiet-flow condition, } \\
& =5 \text { for realistic disturbance level. }
\end{aligned}
$$

The agreement of Eq. 21 with experimentally measured data i.s reasonable, as indicated in Fig. 34, which shows a large discrepancy at low flow velocities. This may be due to mechanically transmitted vibration and to other "system" characteristics, which are overshadowed at higher flow velocities. ${ }^{104}$

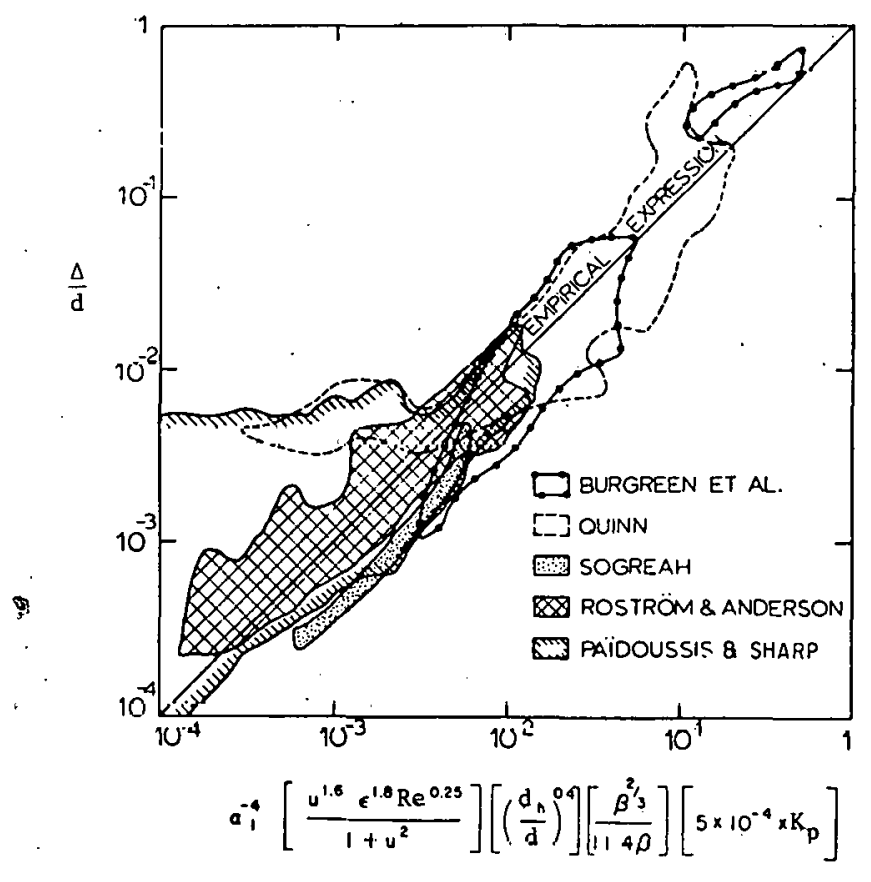

Fig. 34

Agreement between Measured and Piedicted Amplitudes of Vibration according to Paidoussis' Empirical Expression. (From Ref. 104.) 
Since the vibration is random, as discussed below, Paidous sis ${ }^{104}$ addresses the meaning of maximum amplitudes as follows:

"In this empirical expression (Eq. 21) the question arises as to what does the 'maximum' amplitude of vibration $(\Delta)$ represent, recalling that the vibration is random. An answer to this was given by Reavis ${ }^{70}$ who said that it is '... the maximum displacement from equilibrium to be expected if one were to scan through an oscillograph record of rod vibration about $5 \mathrm{ft}$ in length. This length of record corresponds to the author's experience that a data sample is about two arms' lengths long. The author conjectures that the experimenters discussed in this paper, ${ }^{56,105}$ too, scanned vibration-oscillograph records of comparable length.' Although from the theoretical point of view this is an unsatisfactory answer, it is a reasonable one from the practical point of . view."

\section{RMS Displacement Expression}

Wambsganss and Boers ${ }^{71}$ identified the displacement response as a problem in random vibration. In their experiment, the resulting motion was forced-vibration excited by the random pressure fluctuations generated by turbulent eddies in flow. The random-vibration approach was later developed by Reavis, ${ }^{70}$ Gorman, ${ }^{74}$ Chen and Wambsgans s, ${ }^{72,73,75}$ Kanazawa and Boresi, ${ }^{109}$ and Ohlmer et al. ${ }^{110}$ The assumptions made in these methods are as follows: ${ }^{104}$

a. The motion in any one plane is representative of motion as a whole; the random-pressure field is accordingly translated to an equivalent lateral force field.

b. All far-field pressure-fluctuation components are neglected, as well as pressure components arising from turbulence induced by supports, grids, etc., and from boiling and flow-regime changes.

c. No correlation is assumed between the pressure fields on adjacent cylinders (in the case of multicylinder structures).

d. The motion of a cylinder is assumed to have no effect on the pressure field or on the motion of adjacent cylinders.

e. The process is assuiried to be ergodic and the precoure field homogeneous.

f. The cylinder is lightly damped, and response in the first mode of the cylinder is considered to be dominant.

The rms displacement expression developed by Chen and Wambsganss ${ }^{75}$ is perhaps the most rigorous method of those mentioned above. 
They used wall-pressure measurements by Bakewell ${ }^{81}$ on a body of revolution, and those of Clinch, ${ }^{80}$ as well as their own. ${ }^{79}$ Based on the results of a parameter study, a design relationship for estimating the rms displacement of a flexible tube in axial flow was developed: ${ }^{72}$

$$
\operatorname{yrms}(x, U)=\frac{(0.0180) \mathrm{d}^{1.5} \varphi_{1}(\mathrm{x}) \Phi_{0}^{0.5}(\mathrm{U})}{\left(\mathrm{m}_{\mathrm{t}}+\mathrm{m}_{\mathrm{f}}+\mathrm{m}_{\mathrm{i}}\right) \ell^{0.5} \mathrm{f}_{1}^{1.5}(\mathrm{U}) \zeta_{1}^{0.5}(\mathrm{U})}
$$

where

$$
\begin{aligned}
y_{\mathrm{rms}} & =\mathrm{rms} \text { transverse displacement of tube (in.), } \\
\mathrm{U} & =\text { mean axial flow velocity }(\mathrm{ft} / \mathrm{sec}) \\
\varphi_{1}(\mathrm{x}) & =\text { fundamental mode, } \\
& =\varphi_{1}(\mathrm{x})=\sqrt{2} \sin (\pi \mathrm{x} / \ell), \quad 0<\mathrm{x}<\ell \text { for both ends } \operatorname{simply} \text { supported, } \\
& =\varphi_{1}(\mathrm{x})=\cosh (4.73 \mathrm{x} / \ell)-\cos (4.73 \mathrm{x} / \ell)-0.983[\sinh (4.73 \mathrm{x} / \ell) \\
& -\sin (4.73 \mathrm{x} / \ell)], \quad 0<\mathrm{x}<\ell \text { for both ends clamped, }
\end{aligned}
$$

and

$\Phi_{0}=$ intensity of near-field mean-square spectral density of pressure in low-frequency range $\left(1 b^{2}-\sec /\right.$ in. $\left.{ }^{4}\right)$.

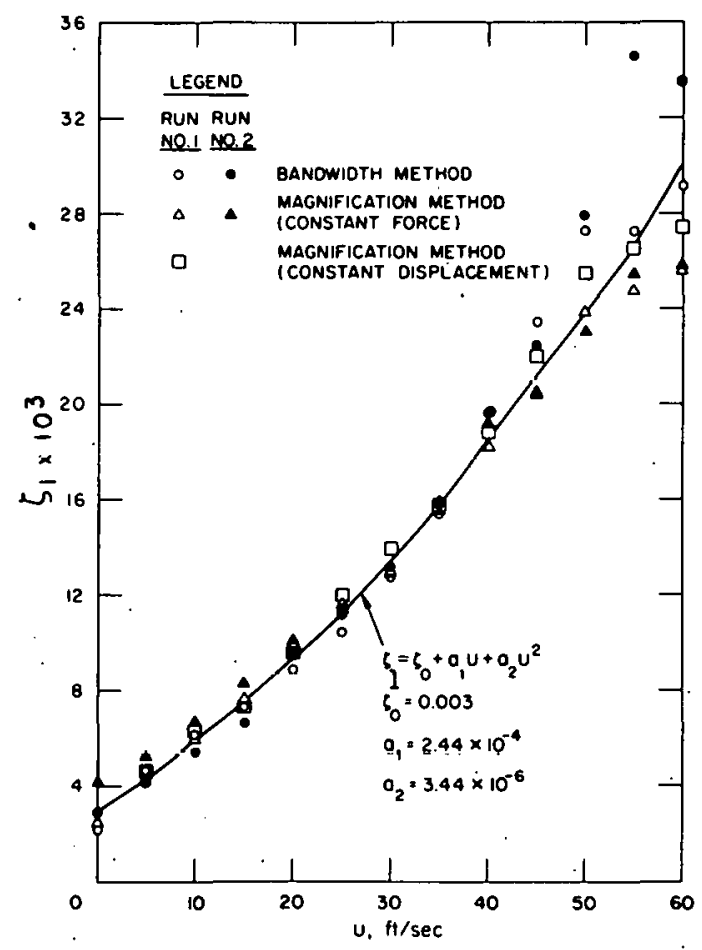

Fig. 35. Equivalent Viscous Damping Factor $\left(\xi_{1}\right)$ as a Function of Mean Axial Flow Velocity (U) for a 0.5-in.-dia, 46-7/8-in.-long Brass Rod with Fixed-Fixed Ends. (From Ref. 72.)
Equation 22 is given in terms of tube natural frequency, damping factor, and the intensity of the mean-square spectrum of the pressure field in the lowfrequency range. All three are functions of mean axial flow velocity. Curves of damping factor and pressure spectra are given in Figs. 35 and $36 .^{72}$ These curves were obtained from measurements on a $1 / 2$-in.-dia rod in a 2 -in.-dia flow channel and can be considered typical of what one can expect from axial flow excitation. Figure 35 shows that, for a given mean axial flow velocity, mean-square spectral density of near-field wall pressure $\left(\Phi_{p}\right)$ is relatively flat out to a "break frequency." Therefore, in the Strouhal-number range $S<2.5$,

$$
\Phi_{\mathrm{p}}(\mathrm{S})=\Phi_{0}(\mathrm{u}) .
$$

In Eq. 22 , effects of fluid viscosity are implicit in the damping $\left(\zeta_{1}\right)$ and pressure spectrum $\left(\Phi_{0}\right)$ terms. In general, the effect of increasing viscosity will be to 
increase the damping. ${ }^{89}$ Viscosity is not expected to have a significant effect on the magnitude of the turbulent wall pressure fluctuations as characterized by $\Phi_{0}$.

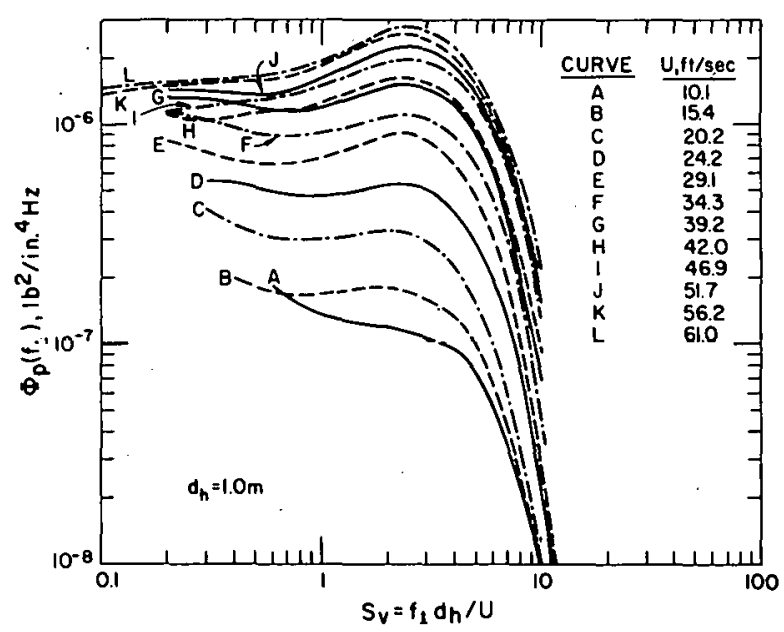

Fig. 36

Family of Near-field Mean-square Wallprcssurc Spectra ( $\left.\Phi_{\mathrm{p}}\right)$ as a Function of Strouhal Number $\left(\mathrm{S}_{\mathrm{v}}\right)$. (From Ref. 72.)

Amplitudes are of primary importance, since the mode of failure most likely will be fretting or wear. However, for those cases in which stresses are of concern, they are easily obtained from displacements given by

$$
\left[\sigma_{y}(x, U)\right]_{r m s}=\frac{E d}{2} \frac{\partial^{2} y_{r m s}(x, U)}{\partial x^{2}}
$$

or, in terms of midpoint displacement, by

$$
\left[\sigma_{y}(x, U)\right]_{\mathrm{rms}}-\frac{\operatorname{Ed} \psi_{1}^{\prime \prime}(\mathrm{x})}{2 \varphi_{1}(\ell / 2)} y_{\mathrm{rIIs}}(l / 2, u),
$$

where a prime denotes differentiation with respect to $\mathrm{x}$. Values for the mode shape $\varphi_{1}$, and its second derivative $\varphi_{1}^{\prime \prime}$, are tabulated in Ref. 111 .

Although the rms displacement response is useful, a complete description of the random signal requires knowledge of the probability law describing the amplitude distribution. The amplitude distribution is found to be a Gaussian distribution. ${ }^{72}$

The agreement of Eq. 22 with experimentally measured data is reasonable, as shown in Fig. 37.

In addition to the expressions to predict the vibration amplitude of a tube in parallel flow, as discussed above, Chen ${ }^{37}$ also developed a parametric vibration model based on the assumption that the fluctuating velocity of the turbulence about the mean velocity excites the flexural vibration of tubes in the same way that periodic changes in the compressive load on a column may cause parametric instabilities. Data on two-phase axial flow are 
limited. However, Pettigrew and Gorman conducted an experiment on a $48-\mathrm{Hz}$ heated cylinder and found that the vibration amplitude was generally largest at steam qualities between 10 and $25 \%{ }^{112}$

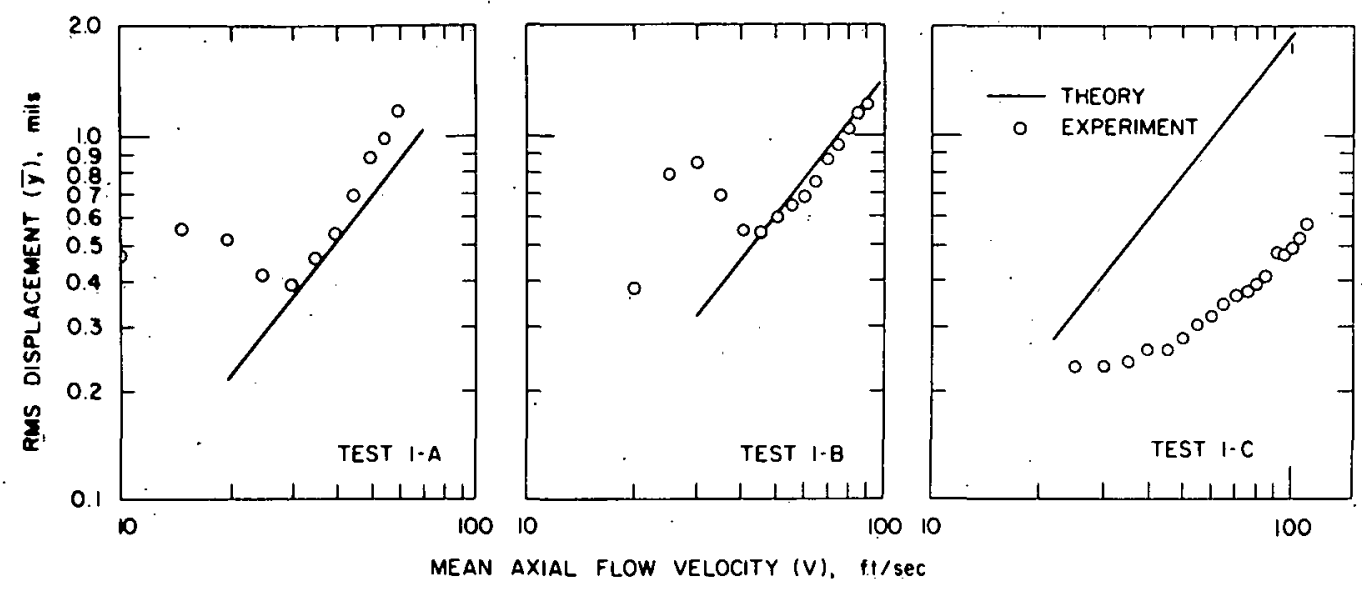

(a) RMS displacement of fixed-fixed rods at $x=(1 / 2) \ell$.

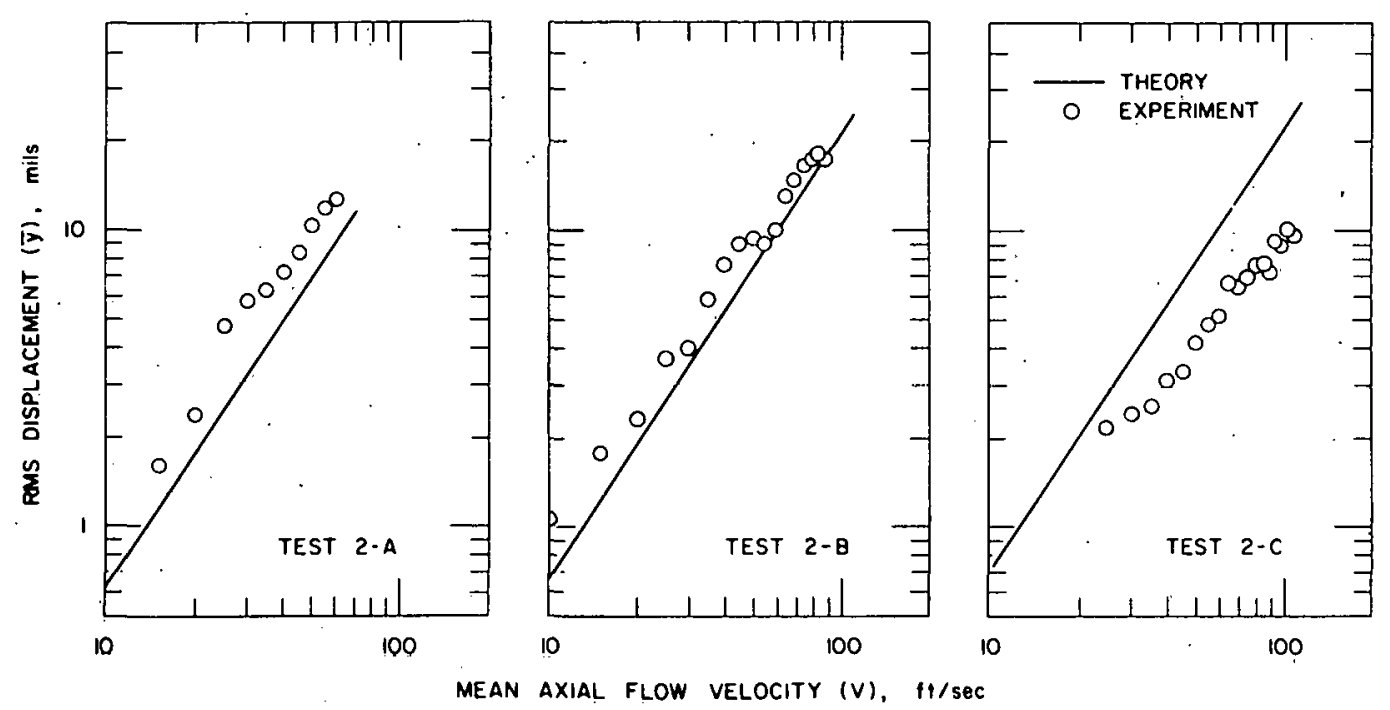

(b) RMS displacement of cantilevered rods at $x=2 \mathrm{ft}$.

Fig. 37. Agreement between Chen and Wambsganss' Theoretical and Experimental Values of the Amplitude of Subcritical Vibration. (From Ref. 75.) 


\section{VIBRATION TESTING}

As discussed in Secs. IV and V, the state of the art of flow-induced vibration analysis is such that, while certain design guidelines are available, methods to predict vibration response are generally lacking. Consequently, the manufacturer must resort to testing to verify that his design is safe from the standpoint of flow-induced vibration. Testing, however, can be very expensive, and consideration must be given to reducing costs as may be possible through the testing of scale models, foreshortened models, or sectors. With an expected design life of $20 \mathrm{yr}$ or more, a different a spect of testing also becomes important: vibration monitoring during operation. As a unit accumulates a large number of hours of operation, internal components may wear (e.g., at support locations) with a resultant change in vibrational characteristics that may, in turn, result in an increased susceptibility to flow-induced vibration.

\section{A. Modol Terting}

To reduce the uncertainties associated with geometric scale modeling, proposed tests generally require the use of prototypic tubes and supports. Because of the cost and inherent difficulties in working with liquid sodium, water is used to simulate shell-side fluid. Therefore, despite the fact that the structural model is prototypic, the test must still be considered a model test, since the liquid sodium is simulated by water. Therefore, consideration must be given to the important simulation parameters. Figure 38 shows the fluid properties of water and liquid sodium as given in Refs. 113 and 114 , respectively.

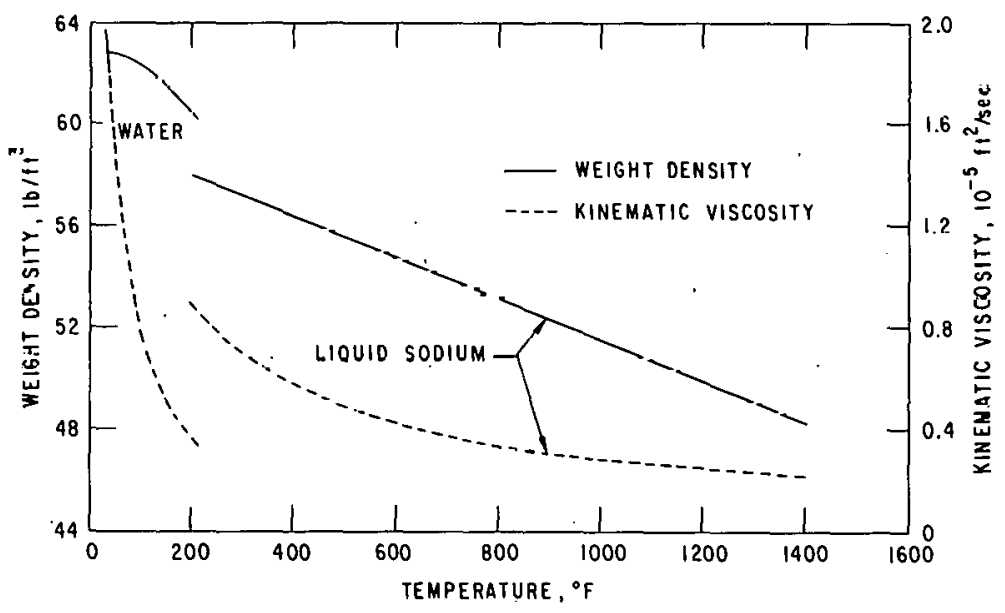

Fig. 38

Flund Density ànd Viscosity vs Temperatilire

The three basic steps in establishing modeling laws from a dimensional analysis are: ${ }^{51}$ (1) selection of the significant independent variables involved in the phenomenon, (2) application of the Buckingham $\pi$ Theorem, and (3) development of similarity requirements by equating $\pi$ terms for prototype and model. A steam-generator hydraulic-model test involves the modeling of continuous structures in incompressible fluid flow. Wright and Bannister ${ }^{115}$ considered this modeling problem. Their selection of independent variables is as follows: 


$$
\begin{aligned}
L= & \text { characteristic length, } \\
\rho_{\mathrm{S}}= & \text { structural mass density, } \\
\eta= & \text { Poisson's ratio, } \\
S= & \text { damping factor, } \\
\mathrm{c}_{\mathrm{S}}= & \text { longitudinal velocity of sound in structure, } \\
\mathrm{F}= & \text { force amplitude, } \\
\omega_{0}= & \text { frequency of driving force (e.g., vortex shedding frequency); } \\
& \text { natural frequency of structure if broad-band random excitation, } \\
\mathrm{U}_{0}= & \text { flow velocity of fluid, } \\
\rho= & \text { mass density of fluid, }
\end{aligned}
$$

and

$$
\nu=\text { kinematic viscosity of fluid. }
$$

Since 10 independent variables have been identified as being significant, by the Buckingham $\pi$ Theorem there are seven (the number of independent variables minus the number of fundamental dimensions) similitude requirements ( $\pi$ terms) for valid dynamic-model tests; these are

$$
\left(\pi_{1}, \pi_{2}, \ldots, \pi_{7}\right)=\left(\frac{\omega L}{c_{s}}, \frac{F}{\rho c_{S}^{2} L}, \eta, \zeta, \frac{\rho_{s}}{\rho}, \frac{U}{c_{s}}, \frac{U L}{\nu}\right) .
$$

Noting that $c_{S}=\sqrt{E / \rho_{S}}$, it can be shown that $\pi_{1}$ represents the ratio of inertia forces to elastic forces, and $\pi_{2}$ represents the ratio of the driving force to the elastic force; $\pi_{3}$ is Poisson's ratio; $\pi_{4}$ is damping; and $\pi_{5}$ is the ratio of material to fluid densities; $\pi_{6}$ can be expressed in the form of a Strouhal number by dividing it by $\pi_{1}$ and taking the inverse to obtain $\omega \mathrm{L} / \mathrm{U}$. Alternatively, $\pi_{6}$ can be expressed in the form of the fluidelastic parameter $\left(\rho U^{2} L^{4}\right) /(E I)$ by using the relationship $c_{s}=\sqrt{E / \rho_{s}}$, introducing $I$ (cross-sectional area moment of inertia), and dividing by $\pi_{5} ; \pi_{7}$ is recognized as the Reynolds number.

Since the velocity of sound in the fluid and the gravitational force are not considered important independent variables for the modeling problem considered, familiar similitude parameters associated with these variables, specifically the Mach number and Froude number, respectively, do not appear among the $\pi$ terms.

In many cases, as with a steam-generator hydraulic-model test, all similarity requirements cannot be satisfied simultaneously. In these cases, the model is said to be distorted. Young ${ }^{116}$ lists three possible procedures for handling distorted models:

1. Neglect certain variables that may be only slightly significant but lead to the distortion, 


\section{Determine the effect of the distortion, analytically, \\ 3. Determine the effect of the distortion, empirically.}

For example, it is generally not possible to simultaneously satisfy Strouhal number $\left(\pi_{6}\right)$ and Reynolds number $\left(\pi_{7}\right)$. Of the two, the Strouhal number is the more important in considering flow-induced vibrations and fluidelastic interaction; the Reynolds number generally is sufficiently high that dynamic fluidelastic phenomena are practically independent of it. ${ }^{115,117}$ For example, as can be seen from Fig. 14, the Strouhal number is independent of Reynolds number over a broad range that includes most steam-generator operating conditions. Nevertheless, Reynolds number should be checked to ensure that "operating conditions" are not in the critical or transition range as defined in Fig. 14.

Development of scaling laws, which account for model distortion and allow the use of model test results to predict prototype behavior, requires an understanding of flow-induced vibration phenumeita. If the functional rclationship between the response and similitude parameters ( $\pi$ terms) is known, a correction factor can be derived to account for the distortion in the model; an example is outlined below.

Consider the problem of using model test results to predict the rms response of steam-generator tubes to parallel flow. The model is assumed to be geometrically similar and to be tested at room temperature. Model distortion is caused by prototypic temperature effects on material and fluid properties and the fact that liquid sodium is simulated by water, which is more dense and has a greater viscosity than liquid sodium. Equation 22 describes the rms displacement of a flexible tube in axial flow. Among other things, the displacement is shown to be proportional to the square root of the intensity of the mean-square spectral density of the near-field wall-pressure fluctuations in the low-frequency range. An experimental study ${ }^{72,79}$ of the wall-pressure fluctuations on the surface of a cylinder in axial flow has shown the frequency distribution of the mean-square pressure $\Phi_{p}$ to be approximately flat out to a cutoff frequency $\left(f_{0}\right)$ as shown in Fig. 36. Consequently, the mean-square pressure can be expressed as.

$$
\left\langle\mathrm{p}^{2}\right\rangle=\int_{0}^{\infty} \Phi_{\mathrm{p}}(\mathrm{f}) \mathrm{df}
$$

or, from the shape of the curve, we can write

$$
\left\langle\mathrm{p}^{2}\right\rangle \approx \Phi_{0} \mathrm{f}_{0}
$$

The rms pressure can be written using an rms pressure coefficient $C_{p}$ defined as

$$
<\mathrm{p}^{2}>^{1 / 2}=\mathrm{C}_{\mathrm{p}}\left(\frac{1}{2} \rho \mathrm{U}^{2}\right)
$$


$\mathrm{C}_{\mathrm{p}}$ has been shown to be approximately constant. ${ }^{79}$ Further, the cutoff frequency $f_{0}$ can be characterized by a Strouhal number (see Fig. 36)

$$
S_{V}=\frac{f_{0} d_{h}}{U}=\text { constant }
$$

Combining Eqs. 25-27 allows us to write

$$
\Phi_{0} \propto \rho^{2} U^{3} d h .
$$

(The parameter grouping on the right-hand side of the proportionality relationship given by Eq. 28 represents a normalization factor employed by many investigators in presenting spectral data on wall-pressure fluctuations; see Ref. 79 for a discussion of its applicability.)

Substituting Eq. 28 into Eq. 22 and rearrranging terms gives

$$
\frac{\mathrm{y}_{\mathrm{rms}}}{\mathrm{L}} \propto \varphi_{1}\left(\frac{\mathrm{L}}{l}\right)^{1 / 2}\left(\frac{\mathrm{d} h}{\mathrm{~L}}\right)^{1 / 2}\left(\frac{\mathrm{L}}{\mathrm{d}}\right)^{1 / 2} \frac{\rho}{\rho_{\mathrm{S}}}\left(\frac{\mathrm{U}}{\omega_{1} \mathrm{~L}}\right)^{3 / 2}\left(\frac{1}{\zeta_{\mathrm{l}}}\right)^{1 / 2}\left[\mathrm{~K}_{0}\left(\frac{\rho}{\rho_{\mathrm{S}}}\right)+1\right]^{-1} \text {. }
$$

where $K_{0}$ is a constant including the added mass coefficient, the ratio of the area of fluid displacement by the cylinder to the cross-sectional area of the cylinder, and the inside cross-sectional area of the tube. As a function of the $\pi$ terms defined by Eq. 23, Eq. 29 takes the form

$$
\frac{y_{\mathrm{rms}}}{\mathrm{L}} \propto \varphi_{\mathrm{n}}\left(\frac{L}{l}\right)^{1 / 2}\left(\frac{\mathrm{d}_{\mathrm{h}}}{\mathrm{L}}\right)^{1 / 2}\left(\frac{L}{\mathrm{~d}}\right)^{1 / 2}\left(\frac{\rho \mathrm{s}}{\rho}\right)^{-1}\left(\frac{\omega_{\mathrm{n}} L}{\mathrm{c}_{\mathrm{s}}}\right)^{-3 / 2}\left(\frac{\mathrm{U}}{\mathrm{c}_{\mathrm{s}}}\right)^{3 / 2} \zeta^{-1 / 2}\left(\mathrm{~K}_{0}\left(\frac{\rho_{\mathrm{s}}}{\rho}\right)^{-1}+1\right)^{-1} \text {. }
$$

The geometry and supports are prototypic in the subject tests; consequently, we can write

$$
\pi_{0}=\frac{\mathrm{y}_{\mathrm{rms}}}{\mathrm{L}} \propto\left[\pi_{1}^{-3 / 2} \pi_{4}^{-1 / 2} \pi_{5}^{-1} \pi_{6}^{3 / 2}\left(\mathrm{~K}_{0} \pi_{5}^{-1}+1\right)^{-1}\right] .
$$

Let $\beta_{\mathrm{n}}$ represent the distortion of the $\mathrm{nth} \pi$ term such that

$$
\begin{aligned}
& \left(\pi_{1}\right)_{\mathrm{m}}=\beta_{1} \pi_{1}, \\
& \left(\pi_{4}\right)_{\mathrm{m}}=\beta_{4} \pi_{4}, \\
& \left(\pi_{5}\right)_{\mathrm{m}}=\beta_{5} \pi_{5},
\end{aligned}
$$

and

$$
\left(\pi_{6}\right)_{\mathrm{m}}=\beta_{6} \pi_{6},
$$

where the subscript $m$ refers to the model. The correction factor $\delta_{1}$ to account for the distortion can then be written 


$$
\delta_{1}=\frac{\pi_{0}}{\left(\pi_{0}\right)_{\mathrm{m}}}=\left(\beta_{1}^{3 / 2} \beta_{4}^{1 / 2} \beta_{5} \beta_{6}^{-3 / 2}\right) \frac{\mathrm{K}_{0} \beta_{5}^{-1} \pi_{5}^{-1}+1}{\mathrm{~K}_{0} \pi_{5}^{-1}+1} .
$$

Similar analyses and computations of correction factors can be carried out to predict prototypic response to other excitation forces, provided the functional relationship between the response and $\pi$ terms is known.

If it can be assumed that the tubes in a steam generator respond primarily as single-span beams between support locations, rather than as beams on multiple supports, then it is generally valid to test foreshortened models, provided a sufficient number of "center" spans are retained to ensure the development of representative flow over a typical section. This reduces the size and cost of the model. However, decreasing the number ul spalls deciedses the modal density (i.e., the number of natural modes to be found in a given frequency range); the consequences of this should be considered.

Flow-induced vibration testing using sectors varying from $120^{\circ}$ to $30^{\circ}$ have been proposed and carried out. Using sectors raises several questions that must be dealt with: these are related to the following:

1. Some steam-generator designs include only one inlet nozzle. Consequently, the inlet flow distribution around the circumference of the unit cannot be expected to be uniform. The problem that arises is to select and test a representative sector out of the available $360^{\circ}$.

2. Testing a sector requires the introduction of solid walls. The effects associated with the presence of solid boundaries must be accounted for.

3. Dependent on design, supports, and flow velocilies, shroud and/or. baffle vibration could be a problem in the prototype. In general, testing of a sector will not allow simulating shroud and baffle dynamics.

In a steam-generator system consisting of evaporator and superheater units, the tube-side flow in the evaporators ranges from single phase (water) at the inlet to two phase (water-steam) at the outlet; in the superheaters, the tube-side fluid is steam. In general, the tube-side flow velocities are sufficiently low that the flow will not act as a significant excitation mechanism, nor will it appreciably affect vibration characteristics of the tubes. In testing a superheater, the tube-side fluid can be neglected on the basis of its density being negligible compared to that of the tube and the added mass of sodium. It might be argued that for an evaporator unit, the tube-side fluid should be simulated by stagnant water to achieve a conservative test from the standpoint of vortex shedding and fluidelastic instability.

In gencral, a test should be designed to allow overtesting, say, by $10-20 \%$, to account for uncertainties in the modeling and to ensure that the design operating conditions are sufficiently separated from any thresholds of instability. As alluded to above, one should also design for conservatism; for 
example, since prototypic values of damping are most often not available, damping in the model should generally be minimized.

B. Surveillance Methods

Because of the adverse environment (high-temperature liquid sodium), vibration monitoring during operation must necessarily rely on external, shellmounted sensors. The problem that arises relates to properly interpreting the measured time histories to determine the dynamic response and or to detect changes in the dynamic response of internal components (e.g., tubes, tube support members, and shrouds) as a function of operating conditions or length of service.

The vibration of a tube within a tube bundle sets up a "disturbance" in the flowing fluid that propagates through the tube bundle to the steam-generator shell. The signal is necessarily attenuated as it propagates through the tube. bundle, and this attenuation must be understood and accounted for in interpreting signals from shell-mounted sensors. Toward this end, Kristiansen and Fahy ${ }^{118}$ reported a theoretical study presenting a simple theory for sound dissipation in crossflow tube banks. In addition to attenuation within the unit, the signal sensed by shell-mounted transducers is also contaminated by extraneous noise associated with turbulence and structure-borne vibrations. Randal1 119 discusses the application of noise-analysis techniques (e.g., cross-spectral densities and coherence) to determine the vibration and impacting of tubes within a heat exchanger. 


\section{CONCLUDING REMARKS}

A. Summary

To avoid flow-induced vibration, the designer can increase the stiffnessto-mass ratios of the structure, reduce flow velocities, and increase damping. However, there is obviously a limit to the extent the se palliatives can be applied since, for example, stiffening the structure implies additional supports, which will increase the pressure drop; and reducing the flow velocities will decrease the heat-removal rate and thus decrease performance. Both minimum flow velocities and maximum allowable pressure drop are fixed from heat-transfer/fluid-flow considerations.

Several excitation mechanisms (e.g., vortex-shedding and fluidelastic whirling) have been identified, and design guidelines are available. To account for uncertainties, it is necessary to allow for a margin of safety. However, designing to give a tube natural frequeñy two to three tirnes greater than the maximum expected vortex-shedding frequency, or designing for a critical flow velocity associated with fluidelastic instability to be two to three times the maximum velocity, will most likely result in an overly conservative design. Such a design has the natural potential for increasing costs and sacrificing performance.

Consequently, optimization of a design with respect to size, cost, and performance, without compromising reliability or safety, requires an in-depth understanding of the excitation mechantsms and lheir cliaracterisation, and the availability of experimentally validated rriethods of analysis. The state-of-theart survey reported in the preceding sections indicates that existing methods of analysis are.inadequate to accurately predict the vibration charactcrietics and response of steam-generator internal components and, therefure, to allow for design optimization. Also, design-verification testing, in general, will not yield information sufficient to assess "how safe" a given design is.

An additional point that deserves emphasis is that the designer should consider the severity of the potential excitation mechanisms and, as possible, should design to avoid the more serious ones. For example, it is recognized that crossflow has a greater potential than parallel flow for exciting tube vibration. Consequently, crossflow regions should be minimized and parallelflow regions designed into the units; as discussed in Sec. II, the more recent LMFBR steam-generator designs reflect this consideration.

In summary, progress is being made in the development of a better understanding of flow-induced vibration phenomena and, consequently, in the development of improved methods of analysis-for example, in the areas of parallel-flow-induced vibration, vortex shedding, and fluidelastic whirling. However, additional base-technology studies are required to resolve critical problem areas and uncertainties associated with present methods of analysis 
and design guidelines and, thereby, to provide for improved predictive design capabilities and the corresponding assurances of reliability and safety of components over their design life. Some specific areas requiring future research are listed in Sec. B below.

\section{B. Future Research Areas}

1. The three dominant excitation mechanisms associated with crossflow-induced vibration of tube arrays are: (a) vortex shedding, (b) fluidelastic whirling, and (c) turbulent buffeting.

Chen ${ }^{37}$ has studied vortex shedding in tube bundles, using results from wind-tunnel tests. While well-defined vortices were measured behind the center tube in a $5 \times 5$-tube array, the effect of bundle size and whether or not "well-organized" vortex shedding even exists deep inside closely spaced tube bundles remains to be established. Chen's tests were performed with rigid tubes, and the effect of fluid/structure interaction on vortex shedding in tube banks requires study. All reported experiments involving tube banks have been designed to achieve, as reasonably as possible, ideal crossflow; the effects of nonuniform and skewed flows have not been reported. With skewed flow, the tube bank can be expected to act as a flow straightener with an accompanying increase in pressure drop, or loss of energy, which may be reflected in the vibration response of the tube.

Connors ${ }^{49}$ demonstrated the fluidelastic whirling vibration of tube rows and bundles that can occur when the crossflow velocity exceeds a critical value. The instability can be characterized by a reduced velocity and a damping parameter, with the "stability boundary" defined by

$$
\frac{V_{T}}{f_{n} d}=K_{T}\left(\frac{m_{0} \delta_{0}}{\rho d^{2}}\right)^{1 / 2}
$$

The proportionality constant $\mathrm{K}_{\mathrm{T}}$ is a.function of system geometry (finned or smooth tubes, and tube spacing) and the number of tubes in a bundle; additional study is required to understand how $\mathrm{K}_{\mathrm{T}}$ is affected by these design variables. Because of the dependence of stability on damping, improved techniques for measuring and estimating damping in tube bundles are required. Also, as can be observed from Fig. 25, with a liquid on the shell side, as contrasted with a two-phase fluid or gas, the critical velocity parameter for vortex shedding is in the same range as that for fluidelastic whirling. The potential for these two excitations to interact and, perhaps, reinforce each other deserves consideration.

In regard to turbulent buffeting within tube banks, additional experimental results are required to evaluate Owen's hypothesis ${ }^{48}$ regarding the existence of a dominant turbulent buffeting frequency, and to determine its relationship, if any, to vortex shedding. 
2. The added mass of fluid and viscous damping are well known for a single cylinder immersed in an infinite fluid medium. The effect of fluid viscosity and annular gap has been recently investigated by Wambsganss and Chen. ${ }^{89}$ However, added mass and the coupling effect of tube rows and bundles remains to be established. Toward this end, Chen is developing a method to compute the added mass-coefficient matrix describing the hydrodynamic forces acting on a given tube in a row or bundle of tubes vibrating in a fluid medium. ${ }^{85,86}$ In matrix format, the diagonal terms are proportional to the hydrodynamic force acting on a given cylinder due to its own acceleration, while the off-diagonal terms represent the coupling and are proportional to the hydrodynamic force due to the acceleration of adjacent tubes in the tube row or bundle.

3. Tube-support thickness, tube-to-support-hole clicarance, Eupporthole shape, support spacing, and misalignment can miakedly affect the vibration characteristics of steam generators. Some of these parameters have received limited study, $2,23,90-9 ?$ the results of which do not yet lend themselvas to prediction of response for a given design. A theoretical analysis relating to tube-baffle impact has been performed. ${ }^{92}$ Although the model was restrictive, it should prove useful in defining the fundamental theoretical and experimental work required to understand and evaluate the effects of variations in the parameters cited above.

4. Shell-side flow-velocity distributions at the inlet-outlet regions, bent-tube regions, and other critical areas must be estimated prior to vibration analysis, since the vibratiun parameters axc cxprcoocd in terms of flow velocities. Currently, there is no established method to estimate flow velocities. Experimental work is needed to investigate flow-velocity distribution as a function of inlet/outlet sizes, tube sizes and spacings, baffle spacing and openings, bypass areás, and other geurnetric parameters, and to dcvclop measurement techniques.

5. Prediction of the vibration rcsponse to flow-induced excitation sources is generally more difficult than prediction of resonant and instability conditions. This is because prediction of vibration response requires mathematical characterization of the fluid-forcing lunction, in addition to satisfactorily modeling fluid/structure interaction and having a knowledge of fluid flow velocities as discussed in items 2-4 above. The oscillating drag and lift forces associated with vortex shedding are generally assumed to be harmunic at frequencies equal to twice and unity, respectively, of the vortex-shedding frequency. The problem is that of selecting the proper value for forcecoefficients; the available experimental data show considerable scatter. Additional information is required on correlation lengths and the interaction effect 3 of tube motion on features related to lift and drag forces. In general, the shell-side flow velocities are of such a magnitude that parallel flow-induced vibration caused by turbulent boundary-layer pressure fluctuations is not a problem. However, additional work would be required to predict response to this excitation source, for example, to evaluate the effect of adjacent tubes and upstream flow disturbances that might be generated by support grids. 
6. Because of the limitations in the development and applicability of state-of-the-art methods of analysis for flow-induced vibrations, model tests are generally relied upon for final design verification. However, even upon completion of hydraulic-model tests, there remain uncertainties and a lack of confidence in applying the test results to predict prototype behavior. Scaling laws must be developed; this, in turn, requires an in-depth understanding of the basic phenomena. involved, as well as knowledge of the functional dependence of the parameter characterizing a particular phenomenon on the similitude requirements.

7: There is a need for improved methods of vibration monitoring and surveillance. For example, methods a re required to account for the attenuation of disturbances associated. with the vibration of tubes within a bundle, and for the use of this information in interpreting results from shell-mounted transducers.

8. Fretting and wear at tube-support locations are one of the more important of the potential failure mechanisms. If one assumes a 30-year life and requires a wear of less than 1/30th of the tube-wall thickness per year, the level of the fretting rate is less than $5 \times 10^{-9} \mathrm{~m} / \mathrm{hr} .^{51}$ The required information relating displacements and forces to wear are not available. The problem is further complicated by an expected "feedback effect" as wear increases clearances.

When sufficient insight and understanding have been gained from ba setechnology studies, such as those suggested above, the applicability of the results to the geometrical configurations and flow patterns of steam generators will have to be confirmed. This will necessitate instrumented flow tests with prototype or model steam generators. As a final step, the results will have to be put into a form (equation or graph) that is readily usable by designers. 


\section{ACKNOW LEDGMENTS}

We wish to thank G. S. Rosenberg for his helpful discussions and contributions and M. R. Sims for his helpful editing suggestions and aid in locating: articles. 


\section{REFERENCES}

1. H. A. Nelms and C: L. Segaser, Survey of Nuclear Reactor System Primary Circuit Heat Exchangers, ORNL-4399 (Apr 1969).

2. T. M. Mulcahy and S. S. Chen, Annotated Bibliography on Flow-induced Vibrations, ANL-CT-74-05 (Jan 1974).

3. A. W. Marris, A Review on Vortex Streets, Periodic Wakes, and Induced Vibration Phenomena, J. Basic Eng. 86(2), 185-196 (1964).

4. J. H. Lienhard, Synopsis of. Lift, Drag and Vortex Fréquency Data for Rigid Circular Cylinders, Bulletin 300, College of Engineering, Washington State University (1966).

5. M. W. Wambsganss, Vibration of Reactor Core Components, Reactor Fuel Process. Technol. 10(3), 208-219 (Summer 1967).

6. P. M. Moretti, A Critical Review of the Literature and Research on FlowInduced Vibrations in Heat Exchangers, AIChE 74th National Meeting, New Orleans, La. (Mar 1973).

7. R. 0. Barratt, J. Polcer, and J. Essebaggers, "High-Integrity Sodium-Heated Steam Generator," ASME Preprint 73-Pwr-14, ASME-IEEE Joint Power Generation Conference, New Orleans, La. (Sept 16-19, 1973).

8. J. P. Petrek, "LMFBR Bayonet Sodium-In-Tube Steam Generator," ASME Preprint 73-Pwr-13, ASME-IEEE Joint Power Generation Conf., New Orleans, La. (Sept 16-19, 1973):

9. Atomic Power Development Associates, Inc., Sodium-Heated Steom Generator Summary, TID-18072 (Sept 1962).

10. G. S. Budney, Liquid-Metal-Heated Stecm Generator Operating Experiences, NAA-SR-12534 (Nov 1967).

11. P. D. Stevens-Guille, Steam Generator Tube Failure: World Experience in Water-Cooled Nuclear Power Reactors During 1972, AECL-4753 (Mar 1974).

12. R. 'H. Anderson, J. A. Ford, and P. S. Linsey, Design, Fabrication, and Preliminary Operation of the Stean Generators for the Enrico Fermi Atomic Power Plant, APDA-307 (Aug 1968).

13. D. J. Rush, General Electric, private communication (Apr 16, 1974).

14. Foster Wheeler Protected Bayonet Tube LMFBR Steam Generator Preliminary Design, ND-74-48 (Aug 30, 1974).

15. R. M. Oliva, Test Report--Development: FBR Steam Generator Tube Vibration Test, TR-095-33-005 (May 1969).

16. M. W. Wambsganss, Evaluation of Potential Tube Vibration in EBR-II Steam Superheaters and Evaporators at Full Power, ANL-7600 (Nov 1969).

17. C. M. He1del, Enrico F'ermi Atomic. Power Plant Steam Generator Experiences, SAN-8002, pp. 30-47 (1964).

18. Sodium-Heated Stecon Generator, RDT Standard E 4-16T (Feb 1974).

19. Standards, 5th ed., Tubular Exchanger Manufacturers Association (1968).

20. Section III: Nuclear Power Plant Components, and Section VIII: Pressure. Voseale, $\Lambda$ SME Boilcr and Pressure Code, American Suclely of Mechantcal Engineers, N.Y. (1974). 
21. J. F. Sebald and W. D. Nobles, "Control of Tube Vibration in Steam Surface Condensers," Proc., American Power Conference, Vol. XXIV, pp. 630-643 (1962).

22. R. T. Hartlen, "Recent Field Experience with Flow-Induced Vibration of Heat Exchanger Tubes," Paper No. 611, Proc., Int. Symp. on Vibration Problems in Industry, Keswick, England (Apr 10-12, 1973).

23. Y. S. Shin, J. A. Jendrzejczyk, and D. E. Sass, Numerical and Experimental Studies on Dynamics of Beam/Stop Impact Model: Preliminary Analysis, ANL-CT-76-6 (to be published).

24. Y. N. Chen, Flow-Induced Vibration and Noise in Tube-Bank Heat Exchanger due to von Karman Streets, J. Eng. Ind. 90(1), 134-146 (Feb 1968).

25. PWR: Vol. 1--Plant Descriptions and Histories, Vol. 2 (Books 1 and 2)-Experiences; BWR: Vol. 1--Plant Descriptions and Histories, Vol. 2 (Books 1 and 2)--Experiences, Nuclear Power Experlence, Inc. (1974).

26. A. Amorosi, ANL, private communication (Oct 15, 1974).

27. H. Schlichting, Boundary Layer l'heory, 4th ed., McGraw-lilil (1960).

28. L. Füppl, Sitzungsberiechte d. k. bayer. Akad. d. Wissensch. Zu Nuchen 1-17 (1913). The Föppl analysis is given in essence as an example in Milne-Thomson, Theoret. Hydrodynomics, 4th ed., Macmillan, p. 366 (1960).

29. A. Roshko, On the Development of Turbulent Wakes from Vortex Streets, NACA Report 1191 (1954).

30. A. Roshko, On the Wake and Drag of Bluff Bodies, J. Aero. Sci. 22(2), 124-132 (1955).

31. D. J. Tritton, Experiments on the Flow Past a Circular Cylinder at Low Reynolds Numbers, J. Fluid Mech. 6(4), 547-567 (Nov 1959).

32. L. S.'G, Knvasznay, Hot Wire Investigation of the Wake Rehind Cylinders at. Low Repynolids Numbers, Proc. Royal Society (London), Series A 138 , $171-190$ (1949).

33. G. D. Birkhoff, A New Theory of Vortex Street, Naliunal Academy of Sciences, Vol. 38, pp. 409-410 (1952):

34. G. D. Birkhoff, Formation of Vortex Streets, J. Appl. Phys. 24(1), 98-103 (195.3).

35. J. S. Humphreys, On a Circular Cylinder in a Steady Wind at Transition Reynolds Numbers, J. Fluid Mech. 9(4), 603-612 (1960).

36. J. W. Schaeffer and S. Eskinazi, An Analysis of the Vortex Street Generated in a Viscous Flui.d, J. Fluid Mech. 6(2), 241-260 (Aug 1959).

37. Y. N. Chen, "Flow-Induced Vibrations in Tube Bundle Heat Exchangers with Cross and Parallel Flow," Symp. on Flow-Induced Vibration in Heat Exchangers, Winter Annua1 Meeting of ASME, New York, pp. 57-58 (Dec 1970).

38. C. W. Van Atta, Exporiments on Vortex-Shedding from Yawed Circular Cyzinders, AIAA J. 6(5), 931-933 (1968).

39. A. König and R. Gregorig, A Criterion for Vibration in Transverse Flow Over a Tube, Part 3: Vibration Experiments in the Nest of Tubes, Chem. Ing. Tech. 40(13), 645-650 (1968); in German (English translation: ORNL-tr-2104). 
40. C. R. Gerlach and F. T. Dodge, "An Engineering Approach to Tube FlowInduced Vibrations," Symp. on Flow-Induced Vibration in Heat Exchangers, Winter Annual Meeting of ASME, New York, pp. 18-26 (Dec 1970).

41. J. P. Den Hartog, Mechanical Vibrations, 4 th ed., McGraw-Hill (1956).

42. J. R. Dale, R. A. Holler, and G. Gross, Flow-Excited Underwater Cable Vibrations, Naval Research Reviews, Naval Air Development Center, Johnsville, Warminster, Pa. (July 1968).

43. P. M. Morse, Vibration and Sound, 2nd ed., McGraw-Hill, p. 398 (1968).

44. J. S. Fitz-Hugh, "Flow-Induced Vibration in Heat Exchangers," Paper No. 427, Proc. Int. Symp. on Vibration Problems in Industry, Keswick, England (Apr 10-12, 1973).

45. W. M. Walker and G. F. S. Reising, Flow-Induced Vibrations in Cross-Flow Heat Exchangers, Chem. Process. Eng. 49(11), 95 (Nov 1968).

46. M. M. Zdravkovich and J. A. Nuttall, on the Elimination of Aerodynomic Noise in a Staggered Tube Bank, J. Sound Vib. 34(2), 173-177 (May 22, 1974).

47. L. J. Cohan and W. J. Deane, Elimination of Destructive, Self-Excited Vibrations in Large, Gas and Oil-Fired Utility. Units, J. Eng. Power 87(2), 223-228 (Apr 1965).

48. P. R. Owen, Buffeting Excitation of Boizer Tube Vibration, J. Mech. Eng. Sci. 7(4), 431-439 (Dec 1965).

49. H. J. Connors, Jr., "Fluidelastic. Vibration of Tube Arrays Excited by Cross Flow," Symp. on Flow-Induced Vibration'in Heat Exchangers, Winter Annual Meeting of ASME, New York, pp. 42-56 (Dec 1970).

50. S. Mirza and D. J. Gorman, "Experimental and Analytical Correlation of Local Driving Forces and Tube Response in Liquid Flow Induced Vibration of Heat Exchangers," 2nd Conference on Stmuctural Mechanics in Reactor Technology, Berlin, CONF730942, Sec. F6/5 (1973).

51. M. J. Pettigrew, AECL, Chalk River, Canada, private communication (May 1975).

52. H. Halle and W. P. Lawrence, "Crossflow-induced Vibration of Cylinders," Reactor Development Program Progress Report: April-May 1973, ANL-RDP-16, pp. 3.10-3.11 (July 9, 1973).

53. H. Halle, B. L. Boers, and M. W. Wambsganss, Fluidelastic Tube Vibration in a Heat Exchanger Designed for Sodium-to-Air Operation, ASME J. Eng. for Power, Paper 75-Pwr-B (1975).

54. R. D. Blevins, "Fluidelastic Whirling of a Tube Row," ASME Paper No. 74PVP-29, Pressure Vessels and Piping Conference, Miami Beach, Florida (June 1974). To be published in ASME Trans. J. Pressure Vessel Technol.

55. B. W. Roberts, Low Frequency Aeroelastic Vibrations in a Cascade of Circular CyZinders, Mech. Eng. Sci. Monograph No. 4, London Inst. Mech. Eng. (Sept 1966).

56. D. Burgreen, J. J. Byrnes, and D. M. Benforado, Vibration of Rods Induced by Water in Parallel Flow, Trans. ASME 80, 991-1003 (1958).

57. M. P. raidoussis, Lynamies of thlexible slender l'ylinders in Axzal Flow, Part I: Theory, J. Fluid Mech. 26(4), 717-736 (Dec 1966). 
58. M. P. Paidoussis, Dynomics of Flexible Slender Cylinders in Axial Flow, Part II: Experiment, J. Fluid Mech. 26(4), 737-751 (Dec 1966).

59. M. P. Paidoussis, Vibration of Flexible Cylinders with Supported Ends, Induced by Axial Flow, Proc. Inst. Mech. Eng. (London) 180(3J), 268-279 (1965-1966).

60. S. S. Chen and G. S. Rosenberg, Vibrations and Stability of a Tube Conveying Fluid, ANL-7762 (Mar 1971).

61. S. S. Chen, Flow-Induced Instability of an Elastic Tube, ASME Paper No. 71-Vibr.-39 (Sept 1971).

62. S. S. Chen, Dynamic Stability of a Tube Conveying Fluid, Paper 8420, Proc. J. Eng. Mech. Div. ASCE 97 (EM5), 1469-1485 (Oct 1971).

63. S. S. Chen, Instability of a Uniformly Curved Tube Conveying Fluid, J. Appl. Mech. 38(4), 1087 (Dec 1971).

64. S. S, Chen, Vibration and Stability of a Uniformly Curved Tube Conveying Fluid, J. Acoust. Soc. Am. 51(1), 223-232 (Jan 1972).

65. S. S. Chen, "Vibrations of Continuous Pipes Conveying Fluid," Int.' Symp. on Flow-Induced Structural Vibrations, Karlsruhe, Germany (Aug 14-17, 1972).

66. S. S. Chen, Flow-Induced In-Plane Instabilities of Curved Pipes, Nuc1. Eng. Des. 23(1), 29-38 (Oct 1972).

67. S. S. Chen, Out-of-PLane Vibration and Stability of Curved Tube Conveying Fluid, J. App1. Mech. 40(2), 362-368 (1973).

68. S. S. Chen, In-Plane Vibration of Continuous Curved Beams, Nucl. Eng. Des. 25, 413-431 (1973).

69. S. S. Chen, "Analys1s of ExLensible Curved Pipes Conveying Fluid," Seventh U. S. National Congress of Applied Mechanics, Boulder, Colorado (June, 1.974).

70. J. R. Reavis, Vibration Coxrelation for Maximun Fuel-Element Displaoomont in Parallel I'urbulent Flow, Nuc1. Sc1. Eng. 38(1), 63-69 (1969).

71. M. W. Wambsganss and B. L. Boers, Parallel-Flow-Induced Vibration of a Cylindrical Rod, ASME Paper No. 68-WA/NE-15 (Dec 1968).

72. M. W. Wambsganss and $S . S$. Chen, Tentative Design Guide for Calculating the Vibration Response of Flexible CyZiriluical Elements in Axial Flow, ANT.-ETD-71-07 (June 1971).

73. S. S. Chen and M. W. Wambsganss, "Response of a Flexible Rod to Near-Field Flow Noise," Froc. Conf. on Flow-Induced Vibrations in Reactor system Components, ANL-7685, pp. 5-31. (May 1970).

74. D. J. Gorman, Role of Turbulence in the Vibration of Reactor Fuel Elements in Liquid Flow, AECL-3371 (May 1969).

75. S. S. Chen and M. W. Wambsganss, FaraZZeZ-Flow-Induced Vibration of Fuel. Rods, Nucl. Eng. Des. 18, 253-278 (1972).

76. G. M. Corcos, Resolution of Pressure in Turbulences, J. Acoust. Soc. Am. 35(1), 192-199 (1963).

77. G. M. Corcos, The Structure of the Turbulent Pressure Fieid in BoundaryLayer Flows, J. Fluid Mech. 18(3), 353-378 (1964). 
78. W. W. Willmarth and C. E. Wooldridge, Measurements of the Fluctuating Pressure at the Wall Beneath a Thick Turbulent Boundary Layer, J. Fluid Mech. 14(.2), 187-210 (1962).

79. M. W. Wambsganss and P. L. Zaleski, "Measurement, Interpretation, and Characterization of Nearfield Flow Noise," Proc. Conf. on Flow-Induced Vibrations in Reactor System Components, ANL-7685, pp. 112-140 (May 1970).

80. J. M. Clinch, Measurements of the Wall Pressure Field at the Surface of a Smooth-Walled Pipe Containing Turbulent Water Flow, J. Sound Vib. 9(3p), 398-419 (1969).

81. H. P. Bakewe11, Jr., TurbuZent WalZ-Pressure Fluctuations on a Body of Revolution, J. Acoust. Soc. Am. 43(6), 1358-1363 (1968).

82. H. H. Schloemer, Effects of Pressure Gradients on Turbulent-BoundaryLayer Walz-Pressure Fluctuations, J. Acoust. Soc. Am. 42(1), 93-113 (1967).

83. J. P. Batham, Pressure Distributions on Circular Cylinders at Critical Reynolds Numbers, J. Fluid Mech. 57(2), 209-228 (1973).

84. D: C. Card, Hydraulic Noise in Reactor Circuit and Loops, and its Effect on Nuclear Fuel Vibration, 73-CSME-80, ASME-CSME Fluids Engineering Conf., Montreal, Canada (May 1974).

85. S. S. Chen, "Vibrations of a Group of Circular Cylindrical Structures in a Liquid," Paper D-2-7, Proc. Third Int. Conf. Struct. Mech. in Reactor Tech., London (Sept 1-5, 1975).

86. S. S. Chen, Vibrations of a Row of Circular Cylinders in a Liquid, ANL-CT-75-34 (Apr 1975).

87. J. T. Thorngren, Predict Exchanger Tube Damage, Hydrocarbon Process: 49(4), 129-131 (Apr 1970).

88. T. Tinker, ShelZ-Side Characteristics of ShelZ-and-Tube Heat Exchangers, Trans. ASME 80(1), 36-52 (Jan 1958).

89. M. W. Wambsganss, S. S. Chen, and J. A. Jendrzejczyk, Added Mass and Domping of a Vibrating Rod in Confined Viscous Fluids, ANL-CT-75-08 (Sept 1974); also submitted for publication in Trans. ASME J. Appl. Mech.

90. P. M. Moretti and R. L. Lowery, Natural Frequencies and Damping of Tubes in SheZz-and-Tube Heat Exchanger, AIChE 74th National Meeting, New Orleans, La. (Mar 1973).

91. G. L. Elliott and R. J. Pick, "Calculation of Natural Frequencies of Heat Exchanger Tubes," Paper No. 422, Proc., Int. Symp. on Vibration Problems in Industry, Keswick, England (Apr 10-12, 1973).

92. S. S. Chen, G. S. Rosenberg, and M. W. Wambsganss, "On Tube-Baffle Impact During Heat Exchanger Tube Vibration," Symp. on Flow-Induced Vibration in Heat Exchangers, Winter Annual Meeting of ASME, New York, pp. 27-35

(Dec 1970).

93. S. P. Timoshenko, Vibration Problems in Engineering, 3rd ed:, D. Van Nostrand, New York (1955).

94. S. 'S. Chen and M. W. Wambsganss, Design Guide for Calculating Natural Frequency of Straight and Curved Beoms on Multiple Supports, ANL-CT-74-06 (June 197/). 
95. R. R. Archer, Smalz Vibrations of Thin Incomplete Circular Rings, Int. J. Mech. Sci. 1(1), 45-56 (1960).

96. L. Meirovitch, Analytical Methods in Vibrations, MacMillan (1967).

97. R. E. D. Bishop and D. C. Johnson, The Mechanics of Vibration, Cambridge University Press, 1960.

98. K. J. Bathe, E. L. Wilson, and F. E. Peterson, SAP1V - Structural Analysis Program for Static and Dynamic Response of Linear Systems, EERC-73-11 (June 1973).

99. Lester S. S. Lee, Vibration of U-Bend Segments of Heat Exchanger Tubes, AECL-3735 (Jan 1971).

100. Lester S. S. Lee, Natural Vibrations of Heat Exchanger Tubes, Proc., Symp. on Applications of Solid Mechanics, University of Waterloo, Canada, pp. 97-118 (June 26-27, 1972).

101. W. Schneider, M. J. Pettigrew, and P. I. Hodge, "Vibration Analysis in the Design of Steam Generating Equipment for the Bruce Nuclear Power Station," Papcr No. 323, Prne., Trll. Symp. on Vibration Probleme in Industry. Keswick, England (Apr 10-12, 1973).

102. G. S. Rosenberg, ANL, private communication (July 1974).

103. J. B. Erskine and W. Waddington, "A Review of Some Tube Vibration Failures in Shell and Tube Heat Exchangers and Failure Prediction Methods," Paper No. 421, Proc., Int. Symp. on Vibration Problems in Industry, Keswick, England (Apr 10-12, 1973).

104. M. P. Paidoussis, The Dynomical Behavior of Cylindrical Structures in Axial Flow, Ann. Nucl. Sci. Eng. 1(2), 83-106 (1974).

105. M. P. Paidoussis, Vibration of Cylindrical Structupes Indused by Axial Elow, J. Eng. Ind. 96(2), 547-552 (May 1974).

106. E. P. Quinn, Vibration of Fuel Rods in Faralzel Flow, GEAP-4059 (Ju1y 1962).

107. M. P. Paldoussis, The Amplitude of Fluid-Induced Vibration of Cyzinders in Axial Flow, AECL-2225 (Mar 1965).

108. M. P. Paidoussis, An Experimental Study of Vibration of Flexible Cylinders Induced by Nominally Axial Flow, Nuc1. Sci. Eng. 35(1), 127-138 (1969).

109. R. M. Kanazawa and A. P. Boresi, "Calculation of the Response of Rods to Boundary Layer Pressure Fluctuations," Proc., Conf. on Flow-induced Vibrations in Reactor System Components, ANL-7685, pp. 47-63 (May 14-15, 1970).

110. E. Ohlmer, S. Russo, and R. Schwemmle, Investigation of an Analytical Model for Paraliel-Flow-Induced kod Vibrations, Nuc1. Eng. Des. 22, 272-289 (1972).

111. R. E. D. Bishop and D. C. Johnson, Vibration Analysis Tables, Cambridge University Press (1956).

112. M. J. Pettigrew and D. J. Gorman, Experimental Studies on Filow Induced Vibration to Support Steam Generator Design; Part I: Vibration of a Heated Cylinder in Two-Phase Axial Flow, Paper No. 424, Proc., Int. Symp. on Vibration Problems in Industry, Keswick, England (Apr 10-12, 1973).

113. R. L. Daugherty and A. C. Ingersoll, Fluid Mechanics, McGraw-Hill (1954). 
114. G. H. Golden and J. V. Tokar, Thermophysical Properties of Sodium, ANL-7323 (Aug 1967).

115. D. V. Wright and R. C. Bannister, Plastic Models for Structural Analysis; Part II: Experiment Design, Shock Vib. Dig. 2(12), 3-10 (Dec 1970).

116. D. J. Young, Basic Principles and Concepts of Model Analysis, Exp. Mech. $11(7), 325-336$ (July 1971).

117. W. A. Lecher, Considerations of Similarity for Hydroelastic Vibrations, Proc. Inst. Mech. Eng. (London) 181(3A), 25-31 (1966-1967).

118. U. R. Kristiansen and F. J.'Fahy, Sound Propagation and Energy Dissipation in Flow Through Arrays of Small Scatterers, J. Sound Vib. 32(2), 189-193 (1974).

119. R. L. Randall, "Application of Noise Analysis Techniques to Study of Flow Induced Vibration in Reactor System Components," Proc., Conf. on Flowinduced Vibrations in Reactor System Components, ANL-7685, pp. 177-204 (May 14-15, 1970). 